\title{
An accurate genetic clock
}

David Hamilton *

*Department of Mathematics, University of Maryland, College Park

\begin{abstract}
Our method for "Time to most recent common ancestor" TMRCA of genetic trees for the first time deals with natural selection by apriori mathematics and not as a random factor. Bioprocesses such as "kin selection" generate a few overrepresented "singular lineages" while almost all other lineages terminate. This non-uniform branching gives greatly exaggerated TMRCA with current methods. Thus we introduce an inhomogenous stochastic process which will detect singular lineages by asymmetries, whose "reduction" then gives true TMRCA. Reduction implies younger TMRCA, with smaller errors. This gives a new phylogenetic method for computing mutation rates, with results similar to "pedigree" (meiosis) data. Despite these low rates, reduction implies younger TMRCA, with smaller errors. We establish accuracy by a comparison across a wide range of time, indeed this is only y-clock giving consistent results for $500-15,000$ ybp. In particular we show that the dominant European y-haplotypes R1a1a \& R1b1a2, expand from c3700BC, not reaching Anatolia before c3300BC. This contradicts current clocks dating R1b1a2 to either the Neolithic Near East or Paleo-Europe. However our dates match R1a1a \& R1b1a2 found in Yamnaya cemetaries of c3300BC by Svante Pääbo et al, together proving R1a1a \& R1b1a2 originates in the Russian Steppes.
\end{abstract}

Molecular clock | Genetic tree | y-haplotype | Kin Selection

Abbreviations: TMRCA, STR, SNP, R1b1a2, R1a1a

\section{Introduction}

$\mathrm{T}$ he genetic clock, computing TMRCA by genetic mutations, was conceived by Emile Zuckerkandl and Linus Pauling[30] on empirical grounds. However work on genetic drift by Motto Kimura[15] gave a theoretical basis and formula. Soon after pioneering work by L.L. Cavalli-Sforza [6], correlated genetic drift to age of lineages for human populations. Suppose at position $j$ on the genome is distinguished by number $x$ which in the next generation has mutation $x \rightarrow x \pm 1$ occurring at rate $\mu_{j}$. Measuring total variance $V$ from the mode [22] one finds that the $\mathrm{TMRCA}=V /\left(\sum_{j} \mu_{j}\right)$. This method and variations (denoted as KAPZ) is used to estimate the TMRCA of $y$ (chromosome) haplotypes defined by a SNP (single nucleotide polymorphism) mutation.

In practice sample sizes were too small to compute accurate mutation rates from "meiosis", i.e. father-son pairs[4]. Alternatively, estimating rates from genetic lineages of known age gave rates with significant discrepancies between different lineages. Indeed for the y-clock these "phylogenetic" rates are often 2 times larger than those from meiosis, while the opposite may be true for other clocks [2], [9], [14], [16].

For the Y-chromosome we show that the mutation rates are essentially constant, at least for the time scale 500- 15,000 ybp, and over different lineages. However KAPZ cannot give accurate TMRCA, i.e. one needs deeper mathematics to deal with non-uniform branching. Also there is a paradox: we can accurately estimate the mutation rates of "short tandem repeat" (STR) at different DNA Y-chromosome Segments (DYS). But we find they can differ by more than a factor of 100 , so over a very long time scale we expect their rates to vary as the genomes geometry changes. Also we find knowing the average mutation rate does not give accurate TMRCA.
Of course it was noticed that the mathematics underlying KAPZ is most accurate for large populations, indeed continuous distributions, whereas actual populations are small. In this case the same stochastic model generates many discrete distributions, indicating a need for Bayesian methods. These use Monte-Carlo simulations of all possible genealogical trees giving the present sample data, then find TMRCA by a maximum likehood estimate (MLE). An example of this for the $y$-clock is BATWING[30]. However we shall see that Bayesian methods exaggerate the TMRCA even more than KAPZ. Also MLE is known for large confidence intervals. So our approach is different.

In particular for the $\mathrm{y}$ (chromosome)-clock the results have not been reliable. (Similar discrepancies occur for the mitrochondrial clock for "out of Africa", or for the allele clock for human-chimpanzee divergence [9], birds [15], bacteria[19].) A KAPZ due to Zhivotovsky [29] was applied to the y-haplotype R1b1a2 by Myres [18] giving 9000BC, standard deviation $\sigma=2000$. Now for BATWING the TMRCA is often greater than KAPZ, e.g. for the Cinnioglu[7] study of Anatolian DNA both methods were applied to the same data and mutation rates. For R1b1a2 the KAPZ has TMRCA 9800BC compared with 18,000BC for BATWING. Balaresque [3] used BATWING to give an origin for R1b1a2 in Neolithic Anatolia

c6000BC, but their statistics was disputed by Busby [5]. In verifying the accuracy of our method we simultaneously resolve the problem of the expansion of European y-haplotypes, for example R1b1a2. 


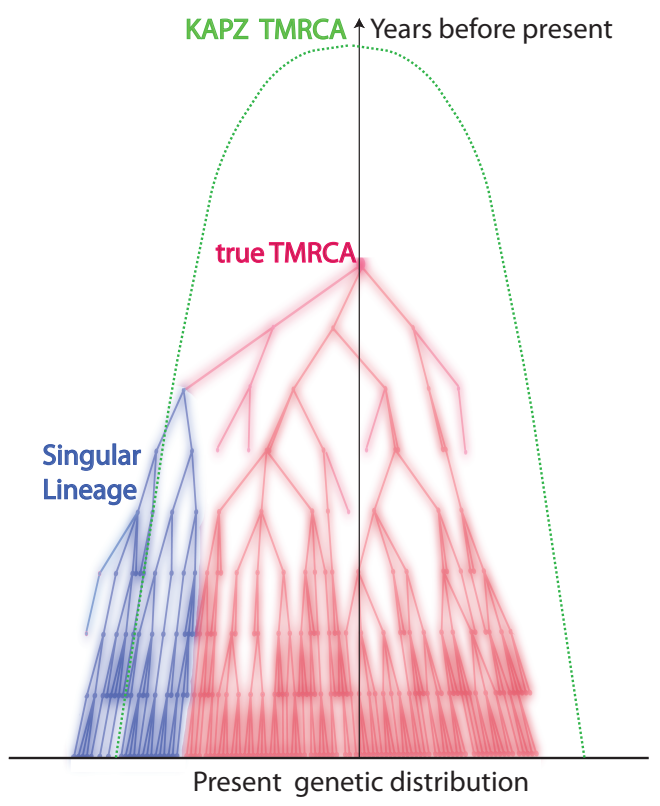

Fig. 1. Random tree

\section{Singular Lineages}

A fundamental problem is that present populations have highly overrepresented branches we call singular lineages. A well known example is the SNP L21 which is a branch of R1b1a2. Individuals identified as L21 are often excluded from R1b1a2 analysis because they skew the results. Such a singular lineage causes the variance to be much greater, even though the original TMRCA remains unchanged, see figure 1.

For Bayesian methods such lineages are very unlikely giving an even greater apparent TMRCA. However one cannot deal with singular branches by excluding them. For one thing, our method will show that $50 \%$ of markers show evidence of singular side branches, i.e. more than a SD from expected. Excluding them would also remove some of the oldest branches and produce a TMRCA which is too young. Now these singular lineages are very (mathematically) unlikely to arise from the stochastic system which is the mathematical basis of KAPZ (or the equivalent Monte-Carlo process modeling BATWING). We believe that the standard stochastic process is perturbed by "improbable" biological processes.

First, the Watson-Galton process[18] implies lineages almost certainly die out. Conversely, natural selection causes some branches to flourish, e.g. the "kin selection" of W.D. Hamilton[13], shows kin co-operation gives genetic advantages. Consider three examples with well developed DNA projects. Group A of the Hamiltons has approximately 100,000 descended from a Walter Fitzgilbert c 1300AD. Group A of the Macdonalds has about 700,000 descendants from Somerfeld c1100AD, and Group A of the O'Niall has over 6 million descendants from Niall of the Seven Hostages, c300AD. These are elite groups with all the social advantages. One sees lines of chieftains, often polygamous. We emphasize kin selection because it seems dominant over natural selection for recent branching, certainly we do not think the O'Niall are genetically superior! Natural selection would cause similar branching over longer time scales. Our model has many extinct twigs with a few successful branches, whereas current models assume a uniform "star radiation".

\section{Reduction of Singular Lineages}

Although our method is for general molecular clocks to be specific we focus on the y-clock. Consider DNA Y-chromosome Segments (DYS) counting the "short tandem repeat" (STR) number of nucleotides. One uses many of these DYS microsatellites, marked by $j=1, \ldots N$, each individual $i, 1=1, . . n$, has STR number $x_{i, j}$. The Y-chromosome is passed unchanged from father to son, except for mutations $x_{i, j} \rightarrow x_{i, j} \pm 1$ occurring at rate $\mu_{j}$.

Modelling singular lineages requires a new stochastic system where instead of a single patriarch we imagine many "virtual patriarchs each originating at a different time and giving a fixed proportion of the present population. Solving for these times and proportions is an inversion problem. But inversion is unstable for such systems, also there is no unique solution. However it turns out that, up to a standard deviation, most DYS markers show at most one singular branch which is found from asymmetries in the distribution. These singular branches are then reduced revealing the original lineage. We then compute a branching time $t_{j}$ for each marker $j$. Now the nonuniform branching process causes the $t_{j}$ to be randomly distributed so their mean is not the TMRCA see figure 2. Large errors in mutation rates means one cannot simply take the $\max t_{j}$ to be the $T M R C A$. Instead stochastic simulations of the branching process, using robust statistics to avoid outliers, find the most likely TMRCA. The effect of reduction is dramatic, e.g. the TMRCA for R1b1a2 changes from $5500 \mathrm{BC}(\mathrm{KAPZ})$ to $3700 \mathrm{BC}$ after singular reduction, using the same markers and mutation rates, see Figure 3 and Table 1.

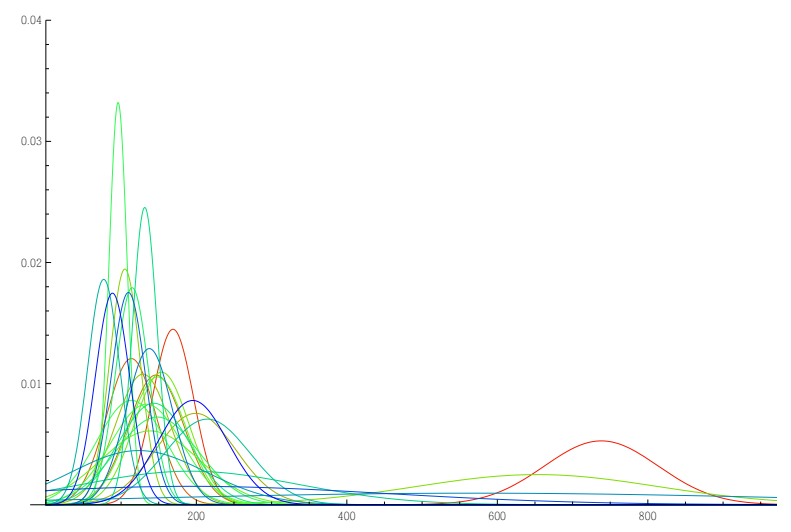

Fig. 2. Branching times $t_{j}$ times(with errors) for R1b1a2 after reduction

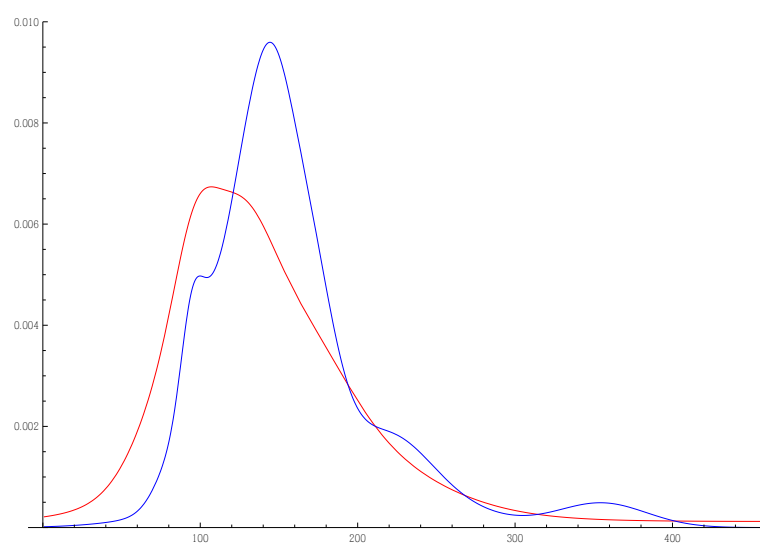

Fig. 3. R1b1a2 branching times before(blue) and after (red) reduction 


\section{Accurate Mutation rates}

By relying on asymmetries of the distribution to find singular lineages we have to be aware that the mutation process itself might not be symmetric. Indeed if ignored we might be just detecting these asymmetries. So the symmetric model has to be changed so the probability of a mutation is

$$
\operatorname{Pr}\left[x_{i, j} \rightarrow x_{i, j}+1\right]=\mu_{j,+1}, \operatorname{Pr}\left[x_{i, j} \rightarrow x_{i, j}-1\right]=\mu_{j,-1} .
$$

If this marker is free from singular lineages we find that the ratio of the frequencies to the left and right of the mode is

$$
\frac{P_{j, 1}(t)}{P_{j,-1}(t)}=\frac{\mu_{j, 1}}{\mu_{j,-1}} \text {. }
$$

which is time independent. So using eight very large SNP projects we find enough markers free of singularities to compute these ratios and their standard deviations. See Supplementary Information (SI) where Figure 5 shows results. In particular about half the markers show asymmetric ratios are significant, i.e more than two SD from ratio 1 . These asymmetric ratios play a very important role, for this ratio is all you need to detect a singular lineage and reduce it. Of course not knowing the exact asymmetric ratio means that bootstrap methods are used extensively for singular reduction, both to compute values and SD.

These methods also imply a new way of computing mutation rates. Previously, there were methods based on meiosos data or phylogenetic studies of family DNA projects (which gave quite different rates). We begin with 8 very large SNP projects from FTDNA using 37 markers, of course with unknown TMRCA. We first reduce singular lineages. Then taking asymmetry into account we find mutation rates are the fixed points of an iterative process. This takes about 3 iterates to converge. These mutation rates are normally distributed with mean and SD. Discarding markers with mutation $S D>33 \%$ leaves us with 29 markers. We find this advanced phylogenetic method gives mutation rates close to those obtained from meiosis and nearly $1 / 2$ the values obtained from the usual phylogenetic method. Further validation comes from finding that the equivalence of our rates with meiosos implies apriori a human generation of c27 years.

\section{Results}

Accuracy is verified by checking for consistency over the whole range of European history beginning with the medieval:

\begin{tabular}{|cccc|}
\hline Group & TMRCA & {$[S D]$} & Origins \\
Hamilton & $1358 A D$ & {$[140]$} & Fitzgilbert d1330AD \\
Macdonald & $900 A D$ & {$[250]$} & Norse $c 800-1000 A D$ \\
$O^{\prime}$ Niall & $200 A D$ & {$[225]$} & Niall 300AD/Conn100AD \\
\hline
\end{tabular}

Archeological finds convinced Marija Gimbutas to attribute Proto Indo-European (PIE) to the Yamnaya Culture c 3500BC of the Russian Steppes, see [12]. This is consistent with mainstream linguistic theory, some even wrote of linguistic DNA. But actual genetics was ignored because current genetic clocks for R1b1a2 pointed to the Renfrew Hypothesis that PIE spread from Neolithic Anatolia, c 6000BC [34]. Or Mesolithic or Paleolithic, depending on the genetic clock. However noone checked if their clock worked over the whole range of time for different lineages.

The next table shows the expansion times of the dominant European y-haplotypes R1b1a2 \& R1a1a. These are very close to $\mathrm{c} 3700 \mathrm{BC}$, only Scandinavia is significantly later. This data is from FTDNA projects for region $\mathrm{X}$ only using individuals with named ancestor from $\mathrm{X}$. These independent results agree within the standard deviation, with dates matching the Corded Ware Culture, a semi-nomadic people with wagons and horses who expanded west from the Urkraine c3500BC. This is consistent with the oldest R1b1a2, R1a1a skeletons being from the Yamnaya Culture, c 3300BC, see S. Pääbo et al $[24]$.

\begin{tabular}{|ccccc|}
\hline Region & $R 1 b 1 a 2[S D]$ & $n$ & $R 1 a 1 a[S D]$ & $n$ \\
All & $3700 B C[625]$ & 460 & $3800 B C[700]$ & 1270 \\
Russia & $N A$ & & $3750 B C[700]$ & 337 \\
Poland & $3960 B C[950]$ & 65 & $4600 B C[820]$ & 876 \\
Germany & $2780 B C[500]$ & 438 & $3750 B C[800]$ & 190 \\
Scandinavia & $2550 B C[500]$ & 153 & $4500 B C[1000]$ & 140 \\
\hline
\end{tabular}

An interesting intermediate step occurs between the medieval and eneolithic. The mythical Irish Chronicles relate that the O'Niall descend directly from the first Gaelic High Kings, which tradition dated c1300-1600BC. The O'Niall have the unique mutation M222 which is a branch of the haplotype L21. For L21, $n=1029$, we compute $T M R C A=1600 B C$ and $S D=320$. These are dates for proto Celtic, i.e. what archeologists call the pre Urnfelder Cultures, c. 1300-1600BC. Furthermore L21 is in turn a branch of haplotype P312 which we date to 2300BC. This date suggests the Bell Beaker Culture of Western Europe. Indeed the only known[24] Bell Beaker genome was found to be P312 with ${ }^{14} C$ date $2300 \mathrm{BC}$.

\begin{tabular}{|ccccc|}
\hline data & Haplotype & $n$ & TMRCA & {$[S D]$} \\
\hline Underhill & R1a1a1 & 974 & $2550 B C$ & {$[400]$} \\
Rootsi & $G 2 a 2$ & 536 & $18500 B C$ & {$[3500]$} \\
\hline
\end{tabular}

Our method requires large data sets and many markers which means we have to rely on data from FTDNA, finding 29 useable markers out of standard 37 they use. In fact many researchers[3] have used FTDNA data. We think our method of reduction with robust statistics solves any problems with this data. To test this we compared our results with R1a1a1

Table 1. Major European SNP: Comparing Singular Reduction for 7, 15, 29 markers with KAPZ. Notice similar TMRCA for KAPZ and Singular Reduction, if there is little branching.

\begin{tabular}{cccccccccc} 
SNP & $\mathbf{n}$ & KAPZ & $<$ SD & $29 \mathrm{mk}$ RSL & $<$ SD & $15 \mathrm{mk}$ RSL & $<$ SD & $7 \mathrm{mk}$ RSL & $<$ SD \\
\hline G2a2b & 1221. & $4840 \mathrm{BC}$ & 1257 & $5359 \mathrm{BC}$ & 900 & $8600 \mathrm{BC}$ & 2120 & $4800 \mathrm{BC}$ & 2050 \\
R1b1a2 & 460. & $5490 \mathrm{BC}$ & 2144 & $3700 \mathrm{BC}$ & 625 & $4300 \mathrm{BC}$ & 950 & $5524 \mathrm{BC}$ & 2000 \\
R1a1a & 1270. & $3670 \mathrm{BC}$ & 1066 & $3800 \mathrm{BC}$ & 700 & $3200 \mathrm{BC}$ & 840 & $3400 \mathrm{BC}$ & 1500 \\
I1 & 2898. & $2400 \mathrm{BC}$ & 1061 & $1800 \mathrm{BC}$ & 400 & $2711 \mathrm{BC}$ & 950 & $3500 \mathrm{BC}$ & 1500 \\
L21 & 1029. & $3270 \mathrm{BC}$ & 1063 & $1600 \mathrm{BC}$ & 325 & $1700 \mathrm{BC}$ & 400 & $1870 \mathrm{BC}$ & 800 \\
U 106 & 1533. & $2530 \mathrm{BC}$ & 628 & $2400 \mathrm{BC}$ & 440 & $2500 \mathrm{BC}$ & 600 & $1800 \mathrm{BC}$ & 800 \\
J 2 & 1241. & $11700 \mathrm{BC}$ & 2990 & $15500 \mathrm{BC}$ & 2600 & $18500 \mathrm{BC}$ & 3000 & $6100 \mathrm{BC}$ & 2100 \\
P 312 & 971. & $2900 \mathrm{BC}$ & 632 & $2240 \mathrm{BC}$ & 420 & $2850 \mathrm{BC}$ & 625 & $2600 \mathrm{BC}$ & 900 \\
\hline
\end{tabular}


data obtained from Underhill[27] with $n=974$ (which involved excluding his four M420 individuals and others with missing markers), and 15 useable markers. The result was $2550 B C$ $\mathrm{SD}=400$, within the CI of our R1a1a results. Table 1 shows the results of extensive simulations using random subsets of our FTDNA data, for 29, 15 and 7 markers. For the same 15 markers as the Underhill[27] the different FTDNA data gives very similar $3300 \mathrm{BC} \mathrm{SD}=840$ for R1a1a, verifying the correctness of using FTDNA data. However once you get down to 7 markers the confidence interval becomes large, e.g. R1a1a gives $3400 B C \mathrm{SD}=1500$. Also it becomes difficult to deal with outliers.

An example with few markers is the R1b1a2 data of Balaresque[3]. Our method (this time with 7 useable markers) gave $\mathrm{SD}>30 \%$. Now Balaresque used the Bayesian method BATWING[30] to suggest a Neolithic origin in Anatolia. With the same Cinnioglu[7] data our method gives for Turkish R1b1a2 $(n=75)$ a TMRCA $=5300 \mathrm{BC}, \mathrm{SD}=3100$, i.e. anytime from the Ice Age to the Iron Age as seen in

\begin{tabular}{|cccc|}
\hline R1b1a2 & $n$ & TMRCA & {$[S D]$} \\
\hline Eire & 75 & $1750 B C$ & {$[1250]$} \\
England & 74 & $1844 B C$ & {$[1250]$} \\
Spain & 207 & $4600 B C$ & {$[1900]$} \\
France & 62 & $4300 B C$ & {$[2400]$} \\
Germany & 147 & $5650 B C$ & {$[2300]$} \\
Turkey & 69 & $5300 B C$ & {$[3100]$} \\
\hline
\end{tabular}

Fortunately, once again, we find good data from FTDNA: the Armenian DNA project, see below. By tradition the Armenians entered Anatolia from the Balkans c1000BC so they might not seem a good example of ancient Anatolian DNA. But some 100 generations of genetic diffusion has resulted in an Armenian distribution of Haplotypes J, G, R1b1a2 closely matching that of all Anatolians, therefore representive of typical Anatolian DNA. We see that Anatolian R1b1a2 arrived after c3300BC, ruling out the Neolithic expansion c6000BC. When dealing with regional haplotypes, e.g. R1b1a2 in Anatolia, the TMRCA is only a upper bound for the arrival times, for the genetic spread may be carried by movements of whole peoples from some other region. This means one has to be careful interpreting regional data, e.g. the TMRCA for the R1b1a2(USA) is c3700BC but nobody thinks it arrived then.

\begin{tabular}{|cccc|}
\hline Armenian & $n$ & TMRCA & {$[S D]$} \\
$R 1 b 1 a 2$ & 99 & $3300 B C$ & {$[800]$} \\
$G 2 a 2 b$ & 46 & $9300 B C$ & {$[2000]$} \\
$J 2$ & 97 & $12100 B C$ & {$[2200]$} \\
\hline
\end{tabular}

Observe that our TMRCA for Armenian G2a2b (formerly G2a3) and J2 show them to be the first Neolithic farmers from Anatolia, i.e. older than $7000 B C$. From Table 1 we see J2, G2a2b for all of Western Europe (non-Armenian data). Our dates show J2 was expanding at the end of the Ice Age. Modern J2 is still concentrated in the fertile crescent, but also in disconnected regions across the Mediterranean. The old genetic model predicted a continuous wave of Neolithic farmers settling Europe [8]. But you cannot have a continuous maritime settlement: it must be leap-frog. Also repeated resettlement from the Eastern Mediterranean has mixed ancient J2 populations, and our method gives the oldest date. On the other hand G2a2b shows exactly the dates expected from a continuous wave of Neolithic farmers across Central Europe. Our dates are consistent with recent findings that the majority of early Neolithic skeletons found in Western Europe are G2a2, c 5000BC see[33], whereas the oldest R1b1a2 found so far is Bellbeaker c2300BC, [24], [25].

\section{Discussion}

Archeology, evolutionary biology, not to mention epidemiology, forensics and genealogy are just some of the applications of molecular clocks. Unfortunately current clocks have been found to give only "ballpark" estimates. Our method is the only one giving accurate time, at least for the human ychromosome verified over the period $500-15,000 \mathrm{ybp}$. There should be many applications for this y-clock, not to mention generalizations to mitochondrial and allele clocks.

Some geneticists thought natural selection makes mutation rates too variable to be useful. The problem is confusion between the actual biochemistry giving mutations and superimposed processes like kin selection producing apparently greater rates. Notice that the SD for our mutation rates is on average $14 \%$ which is much smaller than the actual previous rates. We believe this proves the reality of neutral mutation rates.

Many applications to genetics, forensics, genealogy require the TMRCA between just two individuals, or between two species, a classic method was given by Walsh[28]. While we are accurate for "big data", for this "two -body problem" one cannot determine what singular lineages the branching has been through. Just using our new asymmetric mutation rates will not work. So it would be important to find an accurate method.

Pääbo et al[24], [25] observed all 6 skeletons from Yamnaya sites, c 3300BC by ${ }^{14} C$ dating, are either R1a1b1 and R1a1a. This and other work [33] involve very difficult genetic analysis of specimens which may not always be available. Also such analysis cannot date the origin of R1a1b1 and R1a1a. Our TMRCA shows both these haplotypes expanding at essentially the same time c3700BC. This and our later date for Anatolia, combined with Pääbo et al, implies that R1b1a2 and R1a1a must have originated in the Yamnaya Culture.

In checking accuracy we ran into the question of the origins of PIE. Although there are genes for language there is certainly none for any Indo-European language. Thus inferences have to be indirect. Marija Gimbutas saw patterns in symbolism and burial rituals suggesting the Yamnaya Culture was the cradle of Proto Indo-European. Also their physiology was robustly Europeanoid unlike the gracile skeletons of Neolithic Europe, but this could be nutrition and not genetic. From the above we conclude that the spread of this robust type into Western Europe in the late Neolithic marked an influx of Steppe nomads. Now if R1b1a2 had been shown to spread from Anatolia c6000BC it would have been taken as strong evidence for "out of Anatolia" because of the association of R1b1a2, R1a1 with Indo-European languages. But our accuracy check showed that it was G2a, J2 that spread with the Neolithic Expansion from Anatolia. Now these have been associated with Caucasian languages or Semitic, but never with Indo-European.

\section{Materials and Methods}

This work is biomathematical theory validified by data from publshed sources, see Supplementary Information SI for full mathematical development, data, algorithms and detailed MATHEMATICA worksheets. To verify the theory and compute mutation rates we use diverse data, from FTDNA y-haplotype projects for G2a2b, R1b1a2, R1a, I1, L21, U106, J2, P312. Also we used regional projects for Germany, Scandinavia, Poland and Russia for their R1b1a2, R1a1a data. The Armenian DNA project was important for its R1b1a2, J2 and G2a2b data. We also used DNA projects M222 (O'Niall), Macdonald (Group A which is R1a1a), Hamilton (group A which is I1). This was compared with non FTDNA data from Balaresque, Underhill and Rootsi. 
1. D.W. Anthony, (2007)The Horse, the Wheel and Language, Princeton Univ. Press, Princeton

2. F. J. Ayala et al (2001)Erratic overdispersion of three molecular clocks: GPDH, SOD and XDH, Proc Natl Acad Sci USA vol. 98 no. 20 pp 1140511410

3. P. Balaresque et al (2010) A predominantly Neolithic Origin for European paternal Lineages, PLoS Biol ; 8: e1000285.

4. C. Burgarella et al (2011) Mutation rate estimates for $110 \mathrm{Y}$-chromosome STRs combining population and father-son pair data, European Journal of Human Genetics 19, pp.70- 75; doi:10.1038/ejhg.2010.154

5. G. Busby et al The peopling of Europe and the cautionary tale of $Y$ chromosome lineage R-M269, Philos Trans R Soc Lond. Biol Sci. 2012 Mar 7; 279(1730):884-92. doi: $10.1098 /$ rspb.2011.1044

6. L.L. Cavalli-Sforza et al (2009)Y chromosome diversity, human expansion, drift, and cultural evolution, Proc Natl Acad Sci USA 106, pp. 20174-20179.

7. C. Cinnioglu et al. (2004) Excavating Y-chromosome haplotype strata in Anatolia. Hum Genet. 114, pp.127- 148.

8. C. Edmonds, A.Lillie, L.L.Cavalli-Sforza Mutations arising in the wave front of an expanding population, Proc Natl Acad Sci USA (2004); 10, pp 975-979.

9. N. Elango, J.W. Thomas, S. V. Yi (2006)Variable molecular clocks in hominoids Proc Natl Acad Sci USA vol. 103, no.5,pp13701375

10. C. Epstein, C. and R. Mazzeo(2013)Degenerate Diffusion Operators Arising from Population Biology, Princeton

11. J.N. Fenner, (2005) Cross-Cultural Estimation of the Human Generation Interval for Use in Genetics-Based Population Divergence Studies American Journal of Physical Anthropology128pp 415-428.

12. M. Gimbutas,(1956) The Prehistory of Eastern Europe, Part 1, Cambridge:American School of Prehistoric Research \#20.

13. W.D. Hamilton (1964)The genetical evolution of social behaviour. I, II. Journal of Theoretical Biology 7 (1) pp 1- 52.

14. S.Y. Ho, M.J. Phillips, A. Cooper, A.J. Drummond (2005), Time dependency of molecular rate estimates and systematic overestimation of recent divergence times. Molecular Biology \& Evolution 22 (7): pp 1561-1568.

15. J. Hunt, E. Bermingham, R.Ricklefs, (2001) Molecular systematics and biogeography of Antillean thrashers, tremblers, and mockingbirds Aves: Mimidae. Auk 118 (1)pp. 35 - 55 doi: $10.1642 / 0004-8038$

16. M. Lynch (2010), Rate, molecular spectrum, and consequences of human mutation, Proc Natl Acad Sci USA vol. 107 , no. 3 pp 961968

17. M. Kimura (1968) Evolutionary rate at the molecular level. Nature Vol. 217, pp 624 - 626, doi:10.1038/217624a0

18. D.G. Kendall (1975)The Genealogy of Genealogy Branching Processes before (and after) 1873, Bulletin of the London Mathematical Society 7 (3) pp 225- 253.

19. Andrew H. Knoll et al, (2011), Estimating the timing of early eukaryotic diversification with multigene molecular clocks, Proc Natl Acad Sci USA vol. 108, no. 33, pp 1362413629

20. P. Menozzi, A. Piazza, L.L. Cavalli-Sforza Synthetic maps of human gene frequencies in Europeans. Science(1978) 201:786- 792

21. N. Myre, N. et al A major Y-chromosome haplogroup R1b Holocene era founder effect in Central and Western Europe, European Journal of Human Genetics 19(1) pp 95101

22. Nielsen, R. (Editor)(2005) Statistical Methods in Molecular Evolution, Springer.

23. F. W. J. Olver (1964) Bessel functions of integer order pp. 355- 434 Handbook of Mathematical Functions edited by M. Abramowitz National Bureau of Standards, Washington.

24. S. Pääbo et al (2014), Ancient human genomes suggest three ancestral populations for present-day Europeans, Nature 513, pp 409-413.

25. D. Reich et al (2015) Massive migration from the steppe was a source for IndoEuropean languages in Europe, Nature 14317, doi:10.1038/nature1431

26. S. Rootsi S. et al (2012) Distinguishing the co-ancestries of haplogroup G Y chromosomes in the populations of Europe and the Caucasus, Eur J Hum Genet. 20(12)pp 1275-82. doi: 10.1038/ejhg.2012.86.

27. Peter A Underhill et al, (2015)The phylogenetic and geographic structure of $Y$ chromosome haplogroup R1a , European Journal of Human Genetics 23, pp124-131 doi:10.1038/ejhg.2014.50

28. B. Walsh (2001) Estimating the Time to the Most Recent Common Ancestor for the $\mathrm{Y}$ chromosome or Mitochondrial DNA for a Pair of Individuals, Genetics 158, pp 897-912

29. C.F. Wehrhahn(1975) The evolution of selectively similar electrophoretically detectable alleles in finite natural populations, Genetics 80, pp 375- 394.

30. I. J. Wilson, M.E. Weale, D.J. Balding (2003) Inferences from DNA data: population histories, evolutionary processes and forensic match probabilities.J Roy Statist Soc A. (2003);166:1-33.

31. L.A. Zhivotovsky, L.A. (2000), Estimating Divergence Time with the Use of Microsatellite Genetic Distances, Molecular Biology and Evolution 18, No 5, pp 705-709

32. E. Zuckerkandl and L. Pauling (1965) Evolutionary divergence and convergence in proteins. In Bryson, V.and Vogel, H.J. (editors). Evolving Genes and Proteins. Academic Press, New York. pp. 97-166.

33. M. Lacana et al (2011)Ancient DNA suggests the leading role played by men in the Neolithic dissemination, Proc Natl Acad Sci USA vol. 108, no.45, pp1825518259

34. A.C. Renfrew (1987) Archaeology and Language:The Puzzle of Indo-European Origins, London:Pimlico. 


\section{ONLINE TEXT: Supplementary Information}

\section{Biomathematical theory}

2. Mutation rates tables

3. Mathematica worksheets

4. Data 


\section{Biomathematical theory}

We emphasize the role of extraneous forces like kinselection which operates on too big a scale and rarely enough with results that cannot be subsumed into the mutation rates. So we return to basic principles.

Fundamental Solutions: The Y-chromosome has DYS marked by $j=1, \ldots N$, where one can count the STR number $x_{j}$. Consider the probability $P_{j, k}$ (at time $t$ generations) that at marker $j$ we have $x_{j}=k$. This satisfies the homogenous stochastic system

$$
\frac{P_{j, k}(t)}{d t}=-\mu_{j} P_{j, k}+\sum_{m>0} \mu_{j,-m} P_{j, k-m}+\mu_{j, m} P_{j, k+m}
$$

This homogenous system gives a uniform expansion from a single patriarch.

The system is essentially the model of Wehrhahn[29] who had $\mu_{j,-1}=\mu_{j, 1}$. We introduce asymmetric mutations with total rate

$$
\mu_{j}=\sum_{m>0} \mu_{j,-m}+\mu_{j, m}
$$

About $50 \%$ of DYS markers show asymmetric mutations, i.e. $\mu_{j,-1} \neq \mu_{j, 1}$. tion

The fundamental solution comes from the generator func-

$$
G(z, t)=\sum_{-\infty}^{\infty} P_{j, k} z^{k}
$$

with complex variable $z$, and normalized initial condition $x_{j}=0$ or $P_{j, 0}(0)=1$ :

$$
G(z, t)=\operatorname{Exp}\left[-\mu_{j} t+t \sum_{m>0} \mu_{j,-m} z^{m}+\mu_{j, m} z^{-m}\right]
$$

Then $G$ can be expanded in powers of $z$ to give $P_{j, k}(t)$. Now for the simplest asymmetric case, with only one step mutations, we have $G(z, t)=e^{-\mu_{j} t} e^{t \mu_{j,-1} z} e^{t \mu_{j, 1} / z}=$

$$
e^{-\mu_{j} t}\left\{\sum_{m=0}^{\infty} \frac{\mu_{j,-1}^{m}}{m !}(z t)^{m}\right\}\left\{\sum_{m=0}^{\infty} \frac{\mu_{j,-1}^{m}}{m !}(t / z)^{m}\right\}
$$

so using the Hyperbolic Bessel Function of Order $k \geq 0$, see Olver ${ }^{7}$

$$
I_{k}[u]=\sum_{m=0}^{\infty} \frac{u^{2 m+k}}{2^{2 m+k} m !(m+k) !},
$$

we see that the homogenous system has fundamental solution

$$
P_{j, k}(t)=e^{-\mu_{j} t}\left(\frac{\mu_{j, 1}}{\mu_{j,-1}}\right)^{k / 2} I_{|k|}\left[2 t \sqrt{\mu_{j,-1} \mu_{j, 1}}\right]
$$

From this we obtain the second moment:

$$
\sum_{k=-\infty}^{k=\infty} k^{2} P_{j, k}=\left.\left\{\frac{d}{d z} z \frac{d}{d z} G(z, t)\right\}\right|_{z=1}=t \mu_{j}+t^{2}\left(\mu_{j, 1}-\mu_{j,-1}\right)^{2}
$$

Also from the fundamental solution we find, independently of time

$$
\frac{P_{j, 1}(t)}{P_{j,-1}(t)}=\frac{\mu_{j, 1}}{\mu_{j,-1}}
$$

which we call the asymmetric ratio. It will be repeatedly used.
Of course the actual initial value is not $x_{j}=0$ but was usually taken to be the mode $m_{j}$ which was assumed to be the value for original patriarch. Assuming symmetry, i.e. $\mu_{j,-1}=\mu_{j, 1}$, the TMRCA is:

$$
T=\frac{1}{n \mu} \sum_{j, i}\left(x_{j}(i)-m_{j}\right)^{2}, \mu=\sum_{j} \mu_{j} .
$$

From the present distribution of data we use the frequency

$$
f(j, k)=\frac{\operatorname{Count}\left(x_{j}(i)=k\right)}{n} .
$$

One problem with the KAPZ formula is that higher frequencies $f(j, k),|k|=2,3 \ldots$ are overrepresented in the actual data. This is because the probability of a spontaneous two step mutation is much higher then the product of two one step mutations. So instead we use the frequency to solve the transcendental equation for the unknown $t$

$$
f(j, 0) \sim P_{j, 0}(t)=e^{-\mu_{j} t} I_{0}\left[2 t \sqrt{\mu_{j,-1} \mu_{j, 1}}\right]
$$

This nonlinear equation is easily solved via mathematical software such as MATHEMATICA (I used version 9 running on a boosted 2014 iMac which has accurate hyp erbolic Bessel func-tions. Earlier versions on older iMacs gave inaccuracies so one had to compile one's own functions). Using this formula resolves some other problems with the KAPZ method, e.g. $\mu_{j,-1} \neq \mu_{j, 1}$ gives an extra quadratic term which if ignored causes large errors.

Heterogeneous diffusion equation : However the main problem is singularities in the stochastic process. For a uniform stochastic process, $1-P_{j, 0}(t) \sim 1-f(j, 0)$ is the probability of some mutation. So the expected variance is $f(j, 0)(1-f(j, 0))$. Thus if the actual data variance $V_{j}>>f(j, 0)(1-f(j, 0))$ we are not uniform. Now a sublineage of very high fertility increases variance, giving apparently greater TMRCA although it is unchanged. One finds similar results for Bayesian methods.

The correct approach to nonuniformity assumes at times $t_{i}$ (generations ago) a certain proportion $0 \leq \rho_{i} \leq 1$ of the present population originated from a "virtual patriarch" with an initial STR value $m_{i}$. The resulting system :

$$
\frac{p_{j, k}(t)}{d t}=-\mu_{j} p_{j, k}+\sum_{m>0} \mu_{j,-m} p_{j, k-m}+\mu_{j, m} p_{j, k+m}+d \rho
$$

i.e. $d \rho$ are atoms of weight $\rho_{i}$ with STR value $m_{i}$ occurring at time $t_{i}$. As the system is linear and isotropic the solution is a combination of fundamental solutions $P$ of the homogenous system. Thus the present distribution $f(j, k)$ is

$$
f(j, k)=\sum_{i} \rho_{i} P_{j, k-m_{i}}\left(t_{i}\right)
$$

This allows us to consider populations mixed by having singular lineages from overfertile patriarchs, or by actual immigration from the outside. The inverse problem seeks to find singularities from present data. Unfortunately inversion is ill posed for such systems like the heat equation. This instability produces poor accuracy. Furthermore there is no unique solution, e.g.the present distribution could have been created yesterday.

However we find that $\sim 50 \%$ of the DYS markers show no significant difference from the uniform expansion of a single 
patriarch, i.e. the data variance $V_{j}$ is close to the expected variance $f(j, 0)(1-f(j, 0))$. The other markers show at most one significant side branch, i.e. there is an original branch starting at time $t_{j, 0}$ with STR $m_{0}$ and a second one with STR $m_{1}=m_{0} \pm 1$ at time $t_{j, 1}<t_{j, 0}$ with significant $0<\rho_{1}<\rho_{0}$.

Reduction : We locate these singular lineages by looking for asymmetries in the distribution. For a uniform flow from a single patriarch the frequency of STR value $k$ is given by $f(j, k) \sim P_{j, k}(t)$. The asymmetric ratio:

$$
\frac{f(j, 1)}{f(j,-1)} \sim \frac{P_{j, 1}(t)}{P_{j,-1}(t)}=\frac{\mu_{j, 1}}{\mu_{j,-1}},
$$

is completely independent of time $t$. Therefore if say

$$
\frac{f(j, 1)}{f(j,-1)}>>\frac{\mu_{j, 1}}{\mu_{j,-1}},
$$

we have a singular lineage at $k=+1$. Thus the excess at $k=+1$ is

$$
f(j,+1)-f(j,-1) \frac{\mu_{j, 1}}{\mu_{j,-1}}
$$

To first order approximation then frequency $f(j,+2)$ is due to this singularity at $j=+1$ which therefore gave a contribution

$$
f(j,+2) \frac{\mu_{j,-1}}{\mu_{j,+1}}
$$

to $k=0$. Thus removing the effect of the singularity at $k=+1$ leads to new frequencies

$$
\begin{aligned}
f^{*}(j,-1) & =f(j,-1) \\
f^{*}(j, 0) & =f(j, 0)-f(j,+2) \frac{\mu_{j,-1}}{\mu_{j,+1}} \\
f^{*}(j,+1) & =f(j,+1)-f(j,-1) \frac{\mu_{j, 1}}{\mu_{j,-1}}
\end{aligned}
$$

These of course are no longer normalized so we rescale to obtain the renormalized frequency $F(j, k)$, e.g.

$$
F(j, 0)=\frac{f^{*}(j, 0)}{f^{*}(j, 0)+f^{*}(j,-1)+f^{*}(j,+1)}
$$

which will be used to compute the expansion time for marker $j$. There are similar formulae if the singularity was at $k=-1$.

However there is sampling error both in the frequencies and the $\mu_{j, 1}, \mu_{j,-1}$. So we bootstrap taking into account these uncertainties, running the computation thousands of times. Generally we find the branch singularity is always one of $k=0,+1,-1$ with no SD. In a few cases the singularity may seem to wander between $k=0,+1,-1$. So in the case of a wandering singularity we obtain a distribution over $k=0,+1,-1$ with a mean and SD. In these cases we find the singularity is relatively small and does not make much difference to the final result. However to have a stable method we do not throw out these wandering singularities but in the algorithm use the mean to average between $k=0$ and $k= \pm 1$, e.g. if the mean is $k=0$ then we use the original unreduced frequency.

Notice that we assume at most one side branch. In theory there could be many and solving for these produce even better approximations to the present data. In fact you could get perfect matching but find the atoms were created yesterday! The thing is that while many markers show significant deviation from a uniform flow from a single patriarch, after we have carried out reduction for one possible side branch we find no significant difference from a uniform flow, i.e. the difference is within the SD. This is of course an approximation, the next level beyond Zuckerkandl and Pauling, but given the noise in the data perhaps the best we can do. Later we further reduce the effect of outliers by using robust statistics.

Reducing the singular lineages increases the frequency $f(j, 0)$ of the mode and decreases the computed TMRCA. But as the method of reducing singularities does not respect higher frequencies $f(j, k)$ it follows the KAPZ formula cannot be used and instead we use the probability of no mutations, i.e. solve

$$
F(j, 0)=e^{-\mu_{j} t} I_{0}\left[2 t \sqrt{\mu_{j,-1} \mu_{j, 1}}\right]
$$

This is done for each DYS marker $j$, giving expansion times $t_{1}, \ldots t_{N}$ for each marker, with computed CI. (An extra fixed source of error is the uncertainty in the mutation rates which we deal with later). We find the reduction of singularities makes striking difference to the $t_{j}$ of the effected markers, often a reduction of $\sim 50 \%$ for TMRCA.

Now the existence of side branches implies that the main branch could itself have been the side branch for an earlier branch that did not survive. Thus we do not expect the expansion times $t_{1}, \ldots t_{N}$ for each marker to be essentially equal., i.e they are not within the SD of each other. Indeed we see that the distribution of the times $t_{j}$ for different markers are almost certainly not randomly arranged about a single TRMCA T but distributed from $T$ to the present. This is seen whether you use reduction or not, or our mutation rates or not. (For a given population one could scale mutation rates to get equal $t_{j}$, but then applying these adhoc mutation rates to other populations does not yield the same values). The spread out distribution of surviving branches is another verification of our theory of many extinctions, few survivors. The distribution of the times $t_{j}$ for different markers we call the branching distribution, which is now discussed.

The Branching Distribution : The times $t_{j}$ for different markers are sorted from the youngest to the oldest, forming a sequence $t_{1}^{*}, \ldots t_{N}^{*}$. The generation of these branches is by an unknown probability distribution $d \tau_{0}$ over $[0, T]$. We model $d \tau_{0}$ by assuming a surviving lineage is generated at random with probability $\beta \Delta t$ in time period $[t, t+\Delta t]$, multiplied by the probability that the branching hasn't already occurred. The constant $\beta$ averages fertility and extinction rates, the chance of a new lineage surviving. As $\beta \rightarrow \infty$ we get current theory where all lineages originate from a single patriarch at time $T$. Simulations with the data show that $\beta$ varies in the range 1 to $\infty$. We make no a priori estimate of $\beta$, unlike Bayesian methods where an overall fertility rate is a predetermined parameter. Instead our stochastic simulation will find the most likely $\beta, T$ in each case. Assuming independence, then the generation of branches follows the well known exponential distribution:

$$
\tau_{0}[t]=\operatorname{Exp}[\beta(t-T)] \operatorname{UnitStep}[T-t]
$$

Notice this implies a finite probability that some markers have essentially zero mutations. This is actually seen in examples. Both the Hamilton Gp A and Macdonald Gp A have number of individuals $n>100$. For the time scale of $>700$ years we do not expect there is more than one marker out of 33 which shows absolutely no mutations from the mode. In fact in both cases there are 8 markers where all $n$ individuals have exactly the same STR value. 
Estimating the parameter $T$ for an exponential distribution is a well known problem of statistics. Kendall proved the best estimate for $T$ would be $\max t_{j}$. Unfortunately there is also considerable error $\lambda_{j} \%$ for the mutation rates $\mu_{j}$. Later we give a method for reducing this error, even so we find the SD in the range $10 \%-30 \%$ which gives corresponding range in error for each $t_{j}$. We understand that the $t_{j}$ are being generated by the distribution $d \tau_{0}$ but superimposed on this is a further uncertainty due to mutation rates etc. In particular the largest $t_{j}$ may be wildly inaccurate. Also we found that simply taking the average consistently underestimates the TMRCA by a wide margin.

Assuming the mutation rates have normal distribution with mean $\mu_{j}$ and variance $\lambda_{j}^{2} \mu_{j}^{2}$, the $t_{j}$ have $\operatorname{SD~} t_{j} \lambda_{j}$. Thus the actual data for $t_{j}^{*}$ has probability density function for $s>0$

$$
d \tau(s)=\int_{0}^{T} \frac{e^{(t-T) / \beta}}{\beta} \frac{e^{\frac{-(t-s)^{2}}{2 \nu}}}{\sqrt{2 \pi \nu}} d t .
$$

The variance $\nu$ depends on two sources. First from the uncertainty in mutation rates, for each marker we get variance $\lambda_{j}^{2}$, giving total

$$
\nu_{1}=\frac{1}{N} \sum_{j} \lambda_{j}^{2}
$$

However a small sample also has inherent error from sampling. We are measuring the probability that there is a mutation. This is binomial with probability $H_{j}=H_{j}(t)=$

$$
1-P_{j, 0}(t)=1-e^{-\mu_{j} t}\left(\frac{\mu_{j, 1}}{\mu_{j,-1}}\right)^{k / 2} I_{0}\left[2 t \sqrt{\mu_{j,-1} \mu_{j, 1}}\right]
$$

Hence for sample size $n$ there is variance $H_{j}\left(1-H_{j}\right) / n$, so the variance in time due to this is scaled by the derivative giving:

$$
\nu_{2}=\frac{H_{j}\left(1-H_{j}\right)}{n\left(H_{j}^{\prime}\right)^{2}}
$$

The function $H_{j}^{\prime}$ has actually to be computed as an inverse function depending on $H_{j}$. Therefore the total variance averaged over all $N$ markers is $\nu=\nu_{1}+\nu_{2}$. Although for large samples $(n>1000)$ the second term is insignificant it does effect the results once you get to $n=100$. In our algorithm the branching distribution is used to generate large numbers of random branching times so as to bootstrap error estimates. In turns out much faster to compile the distribution function as a table which can be repeatedly called on.

Estimating TMRCA by Robust Statistics : Inaccurate large values of $t_{j}^{*}$ are mitigated by using "robust" statistics with quintiles instead of means/variances. Using FTDNA data we began with 37 markers. However the 4 markers of DYS464 are unordered and cannot be used. Also we find that markers DYS 19/394, 385b, 459b, CDYb have errors > 33\% in mutation rates so are not used. (These are some of the most popular ones in the literature!). So usually we have $N=29$ markers and take "quintiles" $\theta^{*}=\left(t_{9}^{*}, t_{12}^{*}, t_{15}^{*}, t_{18}^{*}, t_{21}^{*}\right)$. This means that tail end data is not discarded but kept as the information there are 8 values of $t_{j}^{*}>t_{21}^{*}$, which effectively deals with outliers. Bootstrap methods give the confidence interval CI for each quintile.

Thus we wish to find the best estimate of $T$ given $\theta^{*}$ (and CI). This well known statistical problem was investigated by Stochastic Simulations (SS). We also tried Maximum Likehood Methods which gave similar results but with larger CI. Monte-Carlo Methods are used to produce very large numbers $\left(\sim 10^{7}\right)$ of $T, \beta$ with corresponding Distribution. These randomly generate ordered times $\left(s_{1} \ldots s_{29}\right)$ for which we take the quintiles $\theta=\left(s_{9}, s_{12}, s_{15}, s_{18}, s_{21}\right)$. We filter by requiring that $\theta$ close to the data $\theta^{*}$, i.e. $\left\|\theta^{*}-\theta\right\|<2 S D$. This gives a stochastic neighborhood $\mathcal{U}$ of $\theta^{*}$ typically containing $>10^{5}$ sets of data but with $T$ is known for each $\theta \in \mathcal{U}$. Thus we can construct a quasilinear estimator:

$Q L\left(s_{9}, s_{12}, s_{15}, s_{18}, s_{21}\right)=q_{1} s_{9}+q_{2} s_{12}+q_{3} s_{15}+q_{4} s_{18}+q_{5} s_{21}$,

and use least squares over $\mathcal{U}$ to find constants $\left(q_{1}, q_{2}, q_{3}, q_{4}, q_{5}\right)$ minimizing

$$
\left\|q_{1} s_{9}+q_{2} s_{12}+q_{3} s_{15}+q_{4} s_{18}+q_{5} s_{21}-T\right\| .
$$

The $\left(q_{1}, q_{2}, q_{3}, q_{4}, q_{5}\right)$ are computed in MATHEMATICA . We test this by applying the QL to all of $\mathcal{U}$, unsurprisingly

$$
\operatorname{Mean}_{\mathcal{U}}\left[q_{1} s_{9}+q_{2} s_{12}+q_{3} s_{15}+q_{4} s_{18}+q_{5} s_{21}-T\right] \sim 0
$$

What is important is that we find the uncertainty in the SS itself. Actually this depends on the data and is calculated in each case but for our examples we find

$$
S D_{\mathcal{U}}\left[q_{1} s_{9}+q_{2} s_{12}+q_{3} s_{15}+q_{4} s_{18}+q_{5} s_{21}-T\right] \sim .05 T
$$

Finally the quasilinear estimator is applied to the experimental data

$$
\left(t_{9}^{*}, t_{12}^{*}, t_{15}^{*}, t_{18}^{*}, t_{21}^{*}\right)
$$

to obtain our best estimate of $T$. Application of $Q L$ computes the SD for our data, giving part of the overall SD. This must be combined with the SD coming from the uncertainty in the SS. Overall we find that our method has $\mathrm{SD} \sim 12 \%$, this includes variances from our data, mutation rates and uncertainty in the SS. We also tested with 15 and 7 markers. Here one must use "quintiles" $\tau=\left(t_{5}^{*}, t_{8}^{*}, t_{11}^{*}\right), \tau=\left(t_{3}^{*}, t_{5}^{*}\right)$, respectively with all the loss of accuracy that implies. See Table 1 for comparisons using 29, 15, 7 markers on same data.

\section{Accurate Mutation rates:}

Any genetic clock depends on reasonably accurate mutation rates. The meiosos method looks for mutations in father-son studies. However typical rates of $\mu=.002$ would require nearly 50, 000 pairs to get an SD of $10 \%$. Small samples have meant large errors. The phylogenetic approach studies large family groups with well developed DNA/genealogy data. So inverting the KAPZ formula would yield accurate rates. However, singular lineages makes this problematic. Genealogical data might give mutation rates much greater than the biochemical rates because kin selection etc tend to exaggerate the apparent mutation rate. An inspection of 10 different sources finds mutation rates claiming SD $\sim 10 \%$ yet they differ from each other by up to $100 \%$. We describe a new method.

To compute our rates we apply our theory to the large DNA projects for the SNP M222, L21, P312, U106, R1b1a2, I1, R1a1a. This avoids dealing with populations such as family DNA projects which are self selecting, i.e only those with the correct surname which neglects distant branches. Also we have very large samples, our average $n>1000$. Greater accuracy should come from more generations and individuals. The problem is that we do not know their TMRCA. 
Asymmetric Mutation: However before computing mutation rates we must consider asymmetric mutations, i.e. the left and right mutation rates $\mu_{j,-1} \neq \mu_{j, 1}$. For a uniform stochastic process we again use the asymmetric ratio

$$
\frac{p_{j, 1}(t)}{p_{j,-1}(t)}=\frac{\mu_{j, 1}}{\mu_{j,-1}}=\frac{A_{j}}{1-A_{j}}
$$

to define the asymmetric constant $A_{j} \in[0,1]$ for marker $j$. For example $A_{j}=0.5$ is complete symmetry. Of course singularities will effect this ratio, however these only occur $<50 \%$ of markers. Thus for each marker, SNP we compute this ratio. We find the SD for each SNP is relatively small while the difference between SNP can be large. However for each marker, using $8 \mathrm{SNP}$ enables outliers to be easily removed leaving allowing us to use simple linear regression: i.e. average of the $A_{j}$ over the remaining SNP groups. We see that asymmetry is a real effect: $50 \%$ of the $A_{j}$ are more than two SD from symmetry $A_{j}=0.5$.

Observe this is significant. The total second moment is

$$
\sum_{j} \sum_{k=-\infty}^{k=\infty} k^{2} P_{j, k}=t \sum_{j} \mu_{j}+t^{2} \sum_{j}\left(\mu_{j, 1}-\mu_{j,-1}\right)^{2}
$$

So using all our 33 DYS markers with our $\mu_{j}$, we compute constants

$$
\mu=\sum_{j} \mu_{j}=.12006, \tau=\sum_{j}\left(\mu_{j, 1}-\mu_{j,-1}\right)^{2}=0.000236
$$

The KAPZ formula gives variance $V=\mu t$ compared to the corrected formula $\mu t+\tau t^{2}$. The uncorrected KAPZ gives an overestimate $>400 \%$ for $>200$ generations. This effect can be nullified by using the mean instead of the mode, variance instead of the second moment, however failing to do so gives a large error. Furthermore other methods which assume symmetric mutations will also be inaccurate. Having estimates on the asymmetry is essential to our method because we find singular lineages by looking for asymmetry in the data. Any such anomaly needs to be significantly greater than the natural asymmetry.

Mutation Rates as a fixed Point: Next we compute mutation rates using 8 very large SNP groups. First, using the asymmetric constants we find singular lineages and reduce their effect. We take account of the error in the $A_{j}$ by a bootstrap technique, which gives the variance for each frequency $f(j, 0)$. For a given SNP $k$ if markers $j$ started their expansion at the same time TMRCA $T_{k}$ we could calculate mutation rates $\mu_{j}$ via

$$
f(j, 0)=e^{-\mu_{j} T_{j}} I_{0}\left[2 T_{j} \sqrt{\mu_{j,-1} \mu_{j, 1}}\right],
$$

or rather average the 8 different $\mu_{j}$ we would obtain. However because of branching caused by extinction of lineages the different markers do not originate at the same time but at different times $t_{j}$. In this case we expect these $t_{j}$ to be randomly distributed about the log mean over a middle set of times $t_{j}$. So, for each SNP group $k=1, . .8$ define mean time $T_{k}$, not the TMRCA but the mean log mean over a middle set of markers, which is less. We find that this is very stable. So for a fixed marker $j$ the data $\tau_{k, j}=t_{j}-T_{k}$ should be randomly distributed about zero over the different SNP $k=1, . ., 8$. However the wrong choose of $\mu_{j}$ would give a bias. In fact this is what we see if the mutation rates $\mu_{j}=.002$ were chosen. In appendix graphs show the $\tau_{k, j}, k=1, . .8$ bunched around a nonzero point. Thus we try to find $\mu_{j}$ so that the $\tau_{k, j}, k=1,2, . .8$ has mean zero. However the $\tau_{k, j}, k=1,2, . .8$ depend nonlinearly on the rates $\mu_{j}$, as does the mean $T_{k}, k=1, . .8$. We find this nonlinear regression problem is solved by an iterative scheme which starts with any reasonable set of DNA rates, finding any reasonable choice iterates to the same final answer. So choose $\mu_{j}=.002$ to begin. Suppose at some stage we have apparent mutation rates $\mu_{j}$. Then, for each SNP, and each marker we solve equation (1) to obtain the apparent $t_{j}$. For each SNP $k=1, . .8$ we compute the mean $\log$ time $T_{k}$. At the next step we get new rates $\mu_{j}^{*}$ from

$$
f(j, 0)=e^{-\mu_{j}^{*} T_{k}} I_{0}\left[2 T_{k} \sqrt{\mu_{j,-1}^{*} \mu_{j, 1}^{*}}\right]
$$

Averaging $\mu_{j}^{*}, k=1, . .8$ we get our next set of $\mu_{j}$ of mutation rates. However this method would be effected by a marker showing a singular lineage. Fortunately these are few in number and by comparison between the different SNP we remove the outliers. We then repeat the process, computing $T_{k}$ again with the new rates, and another set of mutation rates. So we have an iterative process.

One problem is that the iterates could tend to decrease to zero or increase to $\infty$, as we are only calculating relative rates. To prevent this we renormalize after each iteration so the total $\sum \mu_{j}$ is constant. We found the iterative scheme quickly converges to a fixed set of mutation rates, unique up to a constant factor. The CI is computed by bootstrap parametrized by the uncertainties in data and the asymmetric constants.

The generation factor $\gamma:$ This method does not give absolute mutation rates but relative mutation rates $\mu_{j} \gamma$, where $\gamma$ is universal time scale constant. To find $\gamma$ we apply our method to compute the $T=T M R C A$ of three famous DNA projects and choose $\gamma$ so the scaled $T / \gamma$ best fits the historical record. We choose the DNA projects for the O'Niall(M222), Gp A of Macdonald (R1a1a) and Gp A of the Hamiltons (I1). These are large groups with characteristic DNA and fairly accurate times of origin. Of course finding one constant $\gamma$ from three projects is inherently more accurate than using one project to find 33 different mutation rates. Actually assuming a generation of 27 years these three projects yield $\gamma=1$ with about $5 \%$ error, i.e. there is no actual need for this correction. This is a constant error (like uncalibrated ${ }^{14} C$ dating).

Thus $\gamma$ is related to the length of a generation. Most researchers use 25yrs for $t>500 \mathrm{ybp}$ and 27yrs for $t<500 \mathrm{ybp}$. Balaresque and al used 30yrs based on Fenner [11] who sees a 30yr generation for modern hunter-gatherers. Our theory allows any nominal generation as it really doesn't matter, being included in the $\gamma$ factor which we compute in years not generations. However to give actual mutation rates we need an actual generation so we take 27 years. This appears in our worksheet computation. Notice that choosing a 30 year generation results in a $10 \%$ increase in the quoted mutation rate. As we find our mutation rates are close to the actual rates from meiosis this means the 27 year generation is also correct. 


\begin{tabular}{|c|c|c|c|c|c|c|c|c|}
\hline \# & DYS & Hamilton $^{4}$ & $\mathbf{S D}^{3}$ & Burgella $^{2}$ & $\mathbf{S D}^{3}$ & Chandler $^{2}$ & NIST $^{2}$ & FTDNA $^{4}$ \\
\hline 1. & 393 & 0.72 & 0.14 & 1.03 & 0.36 & 0.76 & 0.08 & 1.43 \\
\hline 2. & 390 & 2.52 & 0.18 & 2.12 & 0.22 & 3.11 & 2.4 & 5.32 \\
\hline 3. & $19 / 394^{1}$ & 1.3 & 0.52 & 2.19 & 0.21 & 1.51 & 2.38 & 1.45 \\
\hline 4. & 391 & 4.98 & 0.2 & 2.72 & 0.18 & 2.65 & 2.88 & 4.15 \\
\hline 5. & $385 \mathrm{a}$ & 1.26 & 0.13 & & & & 2.1 & 5.68 \\
\hline 6. & $385 b^{1}$ & 3.13 & 0.34 & & & & 2.1 & 5.68 \\
\hline 7. & 426 & 0.07 & 0.24 & & & 0.09 & & 0.26 \\
\hline 8. & 388 & 0.22 & 0.22 & 0.42 & 2.31 & 0.22 & & 0.25 \\
\hline 9. & 439 & 3.76 & 0.11 & 5.48 & 0.16 & 4.77 & & 4.95 \\
\hline 10. & 389-I & 1.93 & 0.1 & 2.53 & 0.21 & 1.86 & 1.88 & 2.23 \\
\hline 11. & 392 & 0.36 & 0.27 & 0.43 & 0.59 & 0.52 & 0.58 & 1.59 \\
\hline 12. & $389 \mathrm{~b}$ & 2.96 & 0.11 & 3.17 & 0.18 & 2.42 & 2.96 & 2.72 \\
\hline 13. & 458 & 7.99 & 0.08 & 6.88 & 0.16 & 8.14 & 10.8 & 6.3 \\
\hline 14. & $459 \mathrm{a}$ & 0.39 & 0.18 & & & & & \\
\hline 15. & $459 b^{1}$ & 2.98 & 0.47 & & & & & \\
\hline 16. & 455 & 0.16 & 0.21 & & & 0.16 & & 0.46 \\
\hline 17. & 454 & 0.11 & 0.22 & & & 0.16 & & 0.47 \\
\hline 18. & 447 & 3.8 & 0.15 & 4.56 & 0.96 & 2.64 & & 4. \\
\hline 19. & 437 & 0.99 & 0.18 & & & 0.99 & 1.5 & 2.15 \\
\hline 20. & 448 & 1.16 & 0.21 & & & 1.35 & 1.8 & 2.71 \\
\hline 21. & 449 & 11.7 & 0.14 & 18.97 & 0.52 & 8.38 & & 7.84 \\
\hline 22. & 460 & 2.63 & 0.13 & 3.82 & 0.66 & 4.02 & & \\
\hline 23. & GATAH4 & 3.93 & 0.1 & 2.24 & 0.44 & 2.08 & 2.51 & \\
\hline 24. & YCA IIa & 0.32 & 0.23 & & & & & \\
\hline 25. & YCA IIb & 1.4 & 0.18 & & & & & \\
\hline 26. & 456 & 8.1 & 0.23 & 4.5 & 0.21 & 7.35 & & \\
\hline 27. & 607 & 2.15 & 0.13 & & & 4.11 & & 4.1 \\
\hline 28. & 576 & 10.65 & 0.11 & 16.22 & 0.44 & 10.22 & & 10.2 \\
\hline 29. & 570 & 4.6 & 0.2 & 12.61 & 0.52 & 7.9 & & 7.9 \\
\hline 30. & CDYa & 14.71 & 0.09 & & & & & 35.3 \\
\hline 31. & $\mathrm{CDYb}^{1}$ & 13.4 & 2. & & & & & 35.3 \\
\hline 32. & 442 & 2.9 & 0.11 & & & 3.24 & & \\
\hline 33. & 438 & 0.43 & 0.14 & & & 0.55 & 0.7 & \\
\hline & Mean & 3.6 & & 5.3 & & 3.2 & 2.5 & 6.4 \\
\hline
\end{tabular}

\section{Phylogenetic vs Predigree: MUTATION RATES $\left(\times 10^{-3}\right.$ /generation $)$}

Notes:

1. Too inaccurate to use

2. meiosis: Burgella uses 80 sources, Chandler uses 20

3. $\times 100 \%$ one standard deviation, i.e $\times 2$ for $95 \%$ CI

4. phylogenetic : Reduced Singularities(Hamilton) and not(FTDNA) 


\begin{tabular}{ccccc}
$\#$ & DYS & $\mathbf{A}_{\mathbf{j}}$ & SD & SD to $\mathbf{0 . 5}$ \\
\hline 1. & 393 & 0.675 & 0.087 & 2. \\
2. & 390 & 0.463 & 0.093 & 0.4 \\
3. & $19 / 394$ & 0.973 & 0.032 & 14.7 \\
4. & 391 & 0.029 & 0.008 & 62.8 \\
5. & $385 \mathrm{a}$ & 0.699 & 0.096 & 2.1 \\
6. & $385 \mathrm{~b}$ & 0.82 & 0.085 & 3.8 \\
7. & 426 & 0.37 & 0.232 & 0.6 \\
8. & 388 & 0.91 & 0.072 & 5.7 \\
9. & 439 & 0.734 & 0.359 & 0.7 \\
10. & $389-\mathrm{I}$ & 0.779 & 0.105 & 2.7 \\
11. & 392 & 0.954 & 0.04 & 11.2 \\
12. & $389 \mathrm{~b}$ & 0.703 & 0.325 & 0.6 \\
13. & 458 & 0.512 & 0.137 & 0.1 \\
14. & $459 \mathrm{a}$ & 0.139 & 0.125 & 2.9 \\
15. & $459 \mathrm{~b}$ & 0.003 & 0.001 & 353. \\
16. & 455 & 0.277 & 0.168 & 1.3 \\
17. & 454 & 0.962 & 0.03 & 15.4 \\
18. & 447 & 0.154 & 0.025 & 13.6 \\
19. & 437 & 0.09 & 0.09 & 4.6 \\
20. & 448 & 0.216 & 0.172 & 1.6 \\
21. & 449 & 0.518 & 0.15 & 0.1 \\
22. & 460 & 0.107 & 0.05 & 7.9 \\
23. & GATAH4 & 0.17 & 0.198 & 1.7 \\
24. & YCAIIa & 0.195 & 0.163 & 1.9 \\
25. & YCAIIb & 0.19 & 0.175 & 1.8 \\
26. & 456 & 0.671 & 0.416 & 0.4 \\
27. & 607 & 0.243 & 0.103 & 2.5 \\
28. & 576 & 0.387 & 0.157 & 0.7 \\
29. & 570 & 0.448 & 0.077 & 0.7 \\
30. & CDYa & 0.37 & 0.181 & 0.7 \\
31. & CDYb & 0.258 & 0.082 & 2.9 \\
32. & 442 & 0.603 & 0.17 & 0.6 \\
33. & 438 & 0.715 & 0.215 & 1. \\
\hline Mean* & $\# 1-33$ & $0.26 *$ & 0.134 & \\
& & & &
\end{tabular}

\section{Asymmetric Constants}


Complete worked example for G2a3, R1b1a2, R1a1, I1, L21, U 106, J2, P312.

We use 29 markers (standard method) for G2a3, R1b1a2,R1a1, I1, L21, U106, J2, P312,

requires running compiled functions from 29 ComFun and its data file W29ComFun

First we enter DNA file $\delta \delta$

$\delta \delta \mathrm{x}$

Each file has NN members

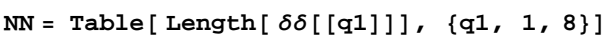

\{NOONI QSMI NOTMI OUVUI NMOVI NRPPI NOQNI VTN\}

We use asymptotic rates $\alpha 0, \beta 0$,LB shown

\begin{tabular}{|c|c|c|c|c|c|c|c|}
\hline MKSTQRVN & MKMUSVQOP & & OKMTPMS & MKUONMRT & & MKTOVMOS & MKPVSMS \\
\hline MKQSPPQS & MKMVORTOR & & MKUSPPVV & MKPONQPR & & -MKNQSUTU & MKPTOOVN \\
\hline MKVTPOQP & MKMPONMUN & & PSKPTPP & QQKUQTP & & PKRVPUP & NKOPOVT \\
\hline MKMOVMMRV & MKMMTRMQTP & & MKMOVUTOP & MKMMTVRVU & & -PKRNMUO & MKOSSQS \\
\hline MKSVUSSN & MKMVSMTVP & & OKPNURO & NKMRUMU & & MKUQMVOU & MKQRSPS \\
\hline MKUOMQRN & MKMURMNN & & QKRSVRN & OKSPSVV & & NKRNVQN & MKRTTMUQ \\
\hline MKPSVSOP & MKOPOORV & & MKRUSPRO & MKRUQQUP & & -MKRPPUPS & MKVVSUNQ \\
\hline MKVMVRSN & MKMTNSUVU & & NMKMRTO & UKTSQUQ & & OKPMUOU & MKUTNRMP \\
\hline MKTPQOUV & MKPRVONQ & & OKTSPQV & RKMUTUT & & NKMNSR & NKUQNN \\
\hline MKTTVPOU & MKNMRNVO & & PKRPNSO & OKNSMNT & & NKOSNTS & MKSNNSSS \\
\hline MKVRQNQN & MKMQMQMNS & & OMKUMRT & NVKONMS & & PKMPROP & MKVOPPP \\
\hline MKTMOTRN & MKPOQUSQ & & OKPSQNU & PKSTSTO & & MKUSMQPN & NKRRRNU \\
\hline MKRNOPSU & MKNPSU & & NKMRMTO & MKRTRPN & & MKMQVQUMO & MKRQTRPS \\
\hline MKNPVQOU & MKNORNOV & & MKNSOMNU & MKNSUVS & & -NKUOMMR & NKMQOUR \\
\hline MKMMPMNOON & MKMMNQMTVV & & MKMMPMONPN & MKMMNQNSRN & & -RKUMOMS & MKQSUUPV \\
\hline MKOTTOQN & MKNSUNNN & & MKPUPRUS & MKPONUNT & & -MKVRUNVN & MKUPUVSV \\
\hline MKVSOQQN & MKMPMMMOR & I & ORKSOQT & ONKOSU & I & PKOQPRS & MKUOVVTV \\
\hline MKNRPRTU & MKMORQPRP & & MKNUNQQQ & MKMPRRMOU & & -NKTMSUN & MKNVRSSV \\
\hline MKMVMMPNO & MKMUVSUMP & & MKMVUVPUU & MKNMUPMQ & & -OKPNPOR & NKMVQSR \\
\hline MKONSOOQ & MKNTOOP & & MKOTRUTQ & MKOUMPSS & & -NKOUTUN & NKMNSOU \\
\hline MKRNUNOP & MKNQVUTU & & NKMTROO & MKSQRQRQ & & MKMTOROQR & MKSMMP \\
\hline MKNMSVUN & MKMQVTMR & & MKNNVTVT & MKMSOPOTQ & & -OKNONVS & MKROMOTR \\
\hline MKNSVRUU & MKNVTVSR & & MKOMQOON & MKOUTMTV & & -NKRUURR & NKQMRTO \\
\hline MKNVQROR & MKNSPQQT & & MKOQNRMP & MKORNVOR & & -NKQOMUT & NKMQPNS \\
\hline MKNUVVTR & MKNTRPSS & & MKOPQRP & MKOSTOT & & -NKQRMNT & NKNPVS \\
\hline MKSTMRSN & MKQNSNRQ & & OKMPRQS & PKUPQQR & & MKTNMTOO & NKUUPUO \\
\hline MKOQPQV & MKNMPOON & & MKPONUS & MKNUMPS & & -NKNPPSQ & MKRSMPST \\
\hline MKPUTPNQ & MKNRTMMR & & MKSPONRT & MKQNUORN & & -MKQRUSNT & MKSSNSOQ \\
\hline MKQQTRO & MKMTTOMNT & & MKUNMMO & MKOROVOS & & -MKONMSVS & MKPNOOQT \\
\hline MKPSVTNO & MKNUMRRV & & MKRUSRTS & MKQRQRMT & & -MKRPPQRQ & MKTTQUQU \\
\hline MKORUQOU & MKMUNUVQR & & MKPQUQUS & MKNQUVNU & & -NKMRQNS & MKQOTPOV \\
\hline MKSMOUOV & MKNTMPPU & & NKRNTU & NKMTVUP & & MKQNTOSR & MKTNNQQO \\
\hline MKTNQSNT & MKONQVS & & OKRMQMS & OKSPVPU & & MKVNTVNR & NKMRQMQ \\
\hline
\end{tabular}

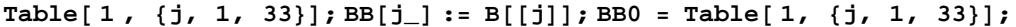

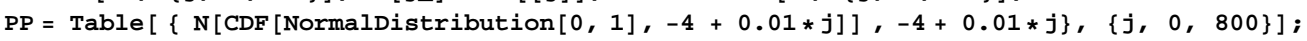

$\mathrm{m}$ = fåíÊêeçä $\sim$ íaçå $[\mathrm{mm}] \mathrm{X}$

We bootstrap with n02 cycles

åMO $=$ NMMM

RMM

The method of reduction is applied 


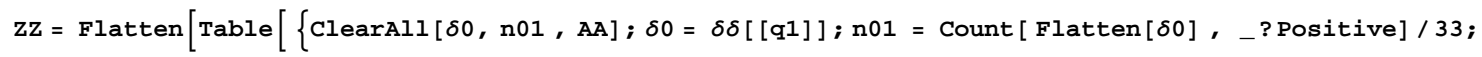

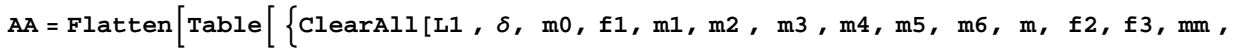

$\delta N I$ ãNMI ãNNI ãNOI ãNPI ãNQI ãNSI ãNTI ãNUI åI oI ooI $\beta I \mu I \alpha I$ eI qpI qpp]X

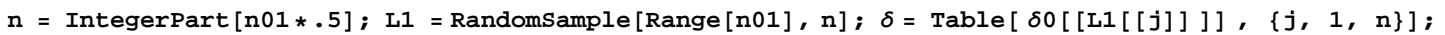

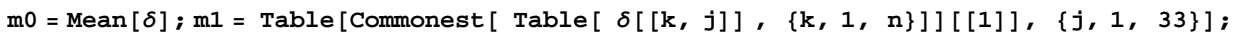

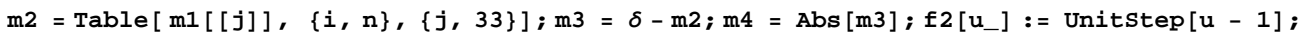

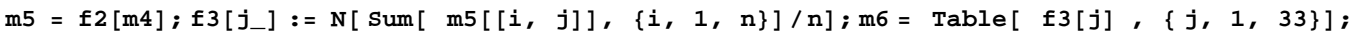

$\tilde{a}=$ q $\ddot{A} \ddot{a} E[$ pìã [ãR[[áI à]] * M[à]I \{àI PP\}]I \{áI å\}] X ãã = k[ pìã [ã [ [á]]I \{áI ås] /å]X

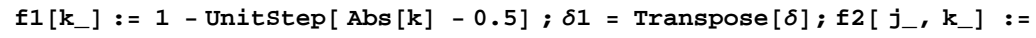

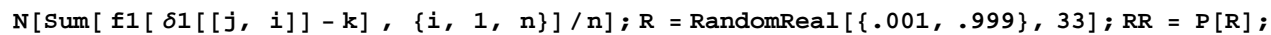

$\beta=$ q $\ddot{A} a ̈ E ́[$ bñé[i_[[àI N]] + i_[[àI O]]*oo[[à]] ]I \{àI NI PP\}]X

$\alpha=q \sim \ddot{A} \ddot{a} E\left[\frac{\beta[[a ̀]]}{N+\beta[[a ̀]]} I\{a ̀ I\right.$ NI PP $]$ X

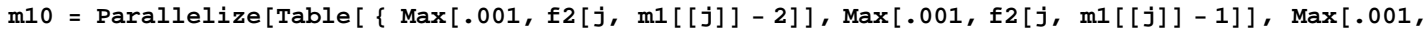
ÑO[àI ãN[[à]]]]I j ñ [KMMNI ÑO[àI ãN[[à]] + N]]I j jn[KMMNI ÑO[àI ãN[[à]] + O]]\}I \{àI NI PP\}]]X

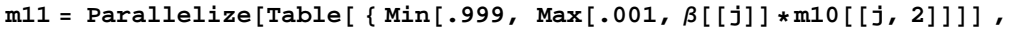

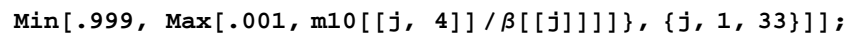

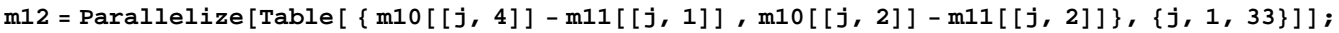

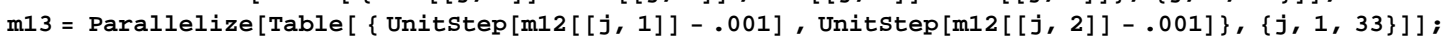

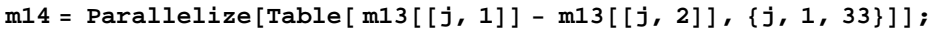

ãNR $=$ m $\sim$ ê $\sim$ ääËăáóÉ [

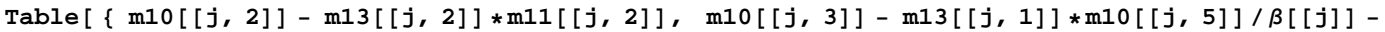
ãNP[[àI O]] * ãNM[[àI N]] * $\beta[$ à]]I ãNM[[àI Q]] - ãNP[[àI N]] *ãNn[[àI N]]\}I \{àI NI PP\}]]X

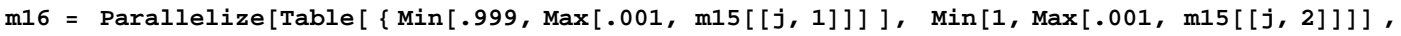

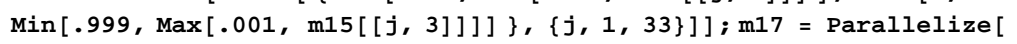

q Ää́́ [ (ãNS[[àI N]] + ãNS[[àI P]]) / (ãNS[[àI O]] + ãNS[[àI N]] + ãNS[[àI P]])I \{àI NI PP\}]]X

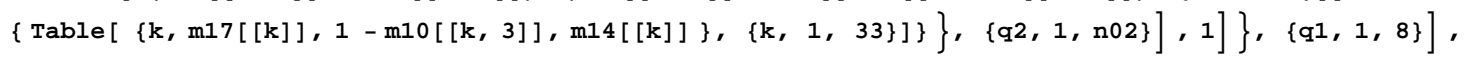

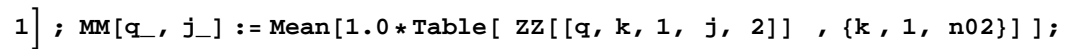

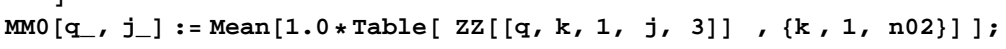

$j j N[e ̀ \mid I$ à|] $W=j E ́ \sim a ̊[N K M * q \sim \ddot{A} a ̈ E ́[$ ww[[èI âI NI àI Q]] I \{âa I NI åMO\}] ]X

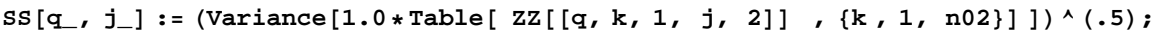

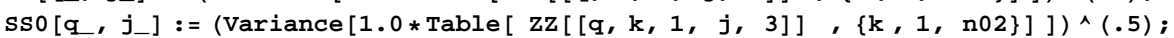

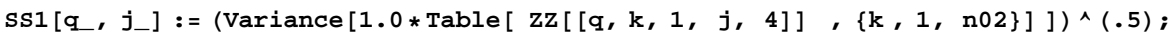

ww $=q \sim \ddot{A} a ̈ E ́[$ [èI àI jj[èI à]I jjM[èI à]I jjN[èI à]I pp[èI à]I ppM[èI à]I ppN[èI à]\}I \{èI NI U\}I \{àI NI PP\}]X

The output is for each file (q), marker (j), reduced frequency f0, unreduced frequency fo, mean \pm then SD for each

j íêáñcçêã [qê åëéçëÉ [ww] ]

\begin{tabular}{|c|c|c|}
\hline $\mathrm{N}$ & 0 & $\mathrm{P}$ \\
\hline $\mathrm{N}$ & $\mathrm{N}$ & $\mathrm{N}$ \\
\hline MKOQMRSU & MKPTUSQO & MKMPTQTRV \\
\hline MKOSVPSQ & MKQMTQT & MKMSNMMQT \\
\hline$-\mathrm{NK}$ & NK & MKSPS \\
\hline MKMNSVMVV & MKMOSOQQT & MKMNNQMOQ \\
\hline MKMNOSOU & MKMOPSUTQ & MKMMSTPQVO \\
\hline MK & MK & MKTQPPTT \\
\hline $\mathrm{N}$ & 0 & $\mathrm{P}$ \\
\hline 0 & 0 & 0 \\
\hline MKNMTNRU & MKONUVSV & MKNUSSVN \\
\hline MKNSSONP & MKPOSRVN & MKOUPVPQ \\
\hline MKTNU & MKOR & -MKUTQ \\
\hline MKMNSVQSP & MKMPMROPP & MKMPOPPQS \\
\hline MKMNNNOSS & MKMONQNOU & MKMNORMNV \\
\hline MKSUSRVV & MKVSQMPP & MKQUQPQU \\
\hline $\mathrm{N}$ & 0 & $P$ \\
\hline$P$ & $P$ & $P$ \\
\hline MKNPNTVT & MKOSPSTR & MKUMNNVS \\
\hline MKNPMO & MKNPPOOS & MKRQSUTO \\
\hline -MKVSO & -MKVPU & $-\mathrm{NK}$ \\
\hline MKMRQQTU & MKOUPNTP & MKNTVRPR \\
\hline MKMMVURRO & MKMNRSSQN & MKMNPOSTT \\
\hline MKOSVSPO & MKPQQMUP & MK \\
\hline $\mathrm{N}$ & 0 & $P$ \\
\hline $\mathrm{Q}$ & $\mathrm{Q}$ & $\mathrm{Q}$ \\
\hline MKNMOMOV & MKQNUTOP & MKRTMQQV \\
\hline MKNMPNMR & MKPSVPSR & MKQVUUQN \\
\hline NK & MKUVS & MKU \\
\hline
\end{tabular}

\begin{tabular}{|c|c|}
\hline Q & $\mathrm{R}$ \\
\hline $\mathrm{N}$ & $\mathrm{N}$ \\
\hline MKMSTTUMP & MKMQVVVVN \\
\hline MKNNUOPT & MKMTQOTSP \\
\hline MKQVS & -MKMQQ \\
\hline MKMNUROPO & MKMNPMQNR \\
\hline MKMMRUQRPQ & MKMMUOOMSP \\
\hline MKURROQT & MKVTNRTO \\
\hline $\mathrm{Q}$ & $\mathrm{R}$ \\
\hline O & 0 \\
\hline MKPSRSNS & MKNTRUMS \\
\hline MKPTRTSV & MKORTQVQ \\
\hline NK & MKUOQ \\
\hline MKMNMMQPV & MKMOQUMRQ \\
\hline MKMMURUNPV & MKMNPPVPU \\
\hline MK & MKRSPSNP \\
\hline Q & $\mathrm{R}$ \\
\hline $\mathrm{P}$ & $\mathrm{P}$ \\
\hline MKNUSUPO & MKMVVQTUS \\
\hline MKONONMO & MKNNMSQO \\
\hline -MKQTQ & -MKPMS \\
\hline MKMVMSSRS & MKMSQPNQP \\
\hline MKMMTSQOS & MKMMVQUTQQ \\
\hline MKUSSRMO & MKUVVVVN \\
\hline Q & $\mathrm{R}$ \\
\hline Q & Q \\
\hline MKMVROST & MKQQSVPT \\
\hline MKMVOVNPT & MKQPPUUT \\
\hline NK & NK \\
\hline
\end{tabular}

$\left.\begin{array}{l}\left(\begin{array}{c}\text { S } \\ \text { N } \\ \text { MKMRPVTRS } \\ \text { MKMUPOSUV } \\ \text {-MKSVU } \\ \text { MKMMVRNRUP } \\ \text { MKMMTNTQQP } \\ \text { MKTMNOT }\end{array}\right) \\ \left(\begin{array}{c}\text { S } \\ \text { O } \\ \text { MKQNSSVT } \\ \text { MKQQRVMN } \\ \text { NK } \\ \text { MKMPROPSR } \\ \text { MKMNOUQUU } \\ \text { MK }\end{array}\right) \\ \text { S } \\ \text { P } \\ \text { MKMUUURUR } \\ \text { MKMUTSROT } \\ \text {-MKSPO } \\ \text { MKMSRSNUR } \\ \text { MKMMTPUMMR } \\ \text { MKTRQTVR }\end{array}\right)$

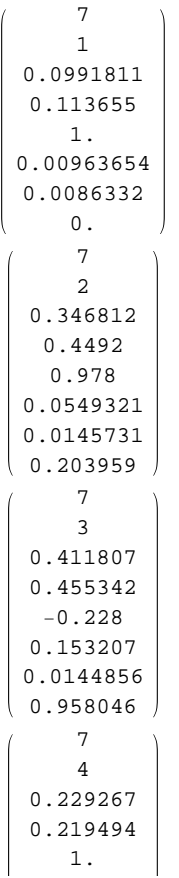

$\left(\begin{array}{c}\text { U } \\ \text { N } \\ \text { MKMTNUQVT } \\ \text { MKNMUNO } \\ \text {-MKQOU } \\ \text { MKMNQVQMT } \\ \text { MKMMVVPPMR } \\ \text { MKUUSTUS }\end{array}\right)$
$\left(\begin{array}{c}\text { U } \\ \text { O } \\ \text { MKNVMMVS } \\ \text { MKOTUUTU } \\ \text {-MKNO } \\ \text { MKMORSRPT } \\ \text { MKMNQUQOU } \\ \text { MKVURSSV }\end{array}\right)$
$\left(\begin{array}{c}\text { U } \\ \text { P } \\ \text { MKNUMSOP } \\ \text { MKNQROMQ } \\ \text {-MKTPQ } \\ \text { MKNSQNVQ } \\ \text { MKMNMVSRT } \\ \text { MKSSVQPP }\end{array}\right)$
U
Q
MKQNRTTP
MKPQOMOR
NK




\begin{tabular}{|c|c|c|c|c|c|c|c|}
\hline $\begin{array}{c}\text { MKMMVUQOUO } \\
\text { MKMMUSPPPP } \\
\text { MK }\end{array}$ & \begin{tabular}{|c|} 
MKNMNTVN \\
MKMONROSP \\
MKQPMTSN
\end{tabular} & $\begin{array}{l}\text { MKOOOQMR } \\
\text { MKMNMSTO } \\
\text { MKRUPSTV }\end{array}$ & $\begin{array}{c}\text { MKMMRURROS } \\
\text { MKMMRPOVQV } \\
\text { MK }\end{array}$ & $\left(\begin{array}{c}\text { MKMOMUMSN } \\
\text { MKMNRTVNP } \\
\text { MK }\end{array}\right)$ & $\begin{array}{c}\text { MKMOTOMRO } \\
\text { MKMNORTU } \\
\text { MK }\end{array}$ & $\begin{array}{c}\text { MKMNRVQNN } \\
\text { MKMNNUMO } \\
\text { MK }\end{array}$ & \begin{tabular}{|c} 
MKMTPVSUN \\
MKMNROSVT \\
MK
\end{tabular} \\
\hline $\mathrm{N}$ & 0 & $P$ & $Q$ & $\mathrm{R}$ & S & $\mathrm{T}$ & $\mathrm{U}$ \\
\hline $\mathrm{R}$ & $\mathrm{R}$ & $\mathrm{R}$ & $\mathrm{R}$ & $\mathrm{R}$ & $\mathrm{R}$ & $\mathrm{R}$ & $\mathrm{R}$ \\
\hline MKPTOVPO & MKNRSRRR & MKNOVQSO & MKPQPSQR & MKMTOORRS & MKMSSUVOV & MKPPMVVV & MKNMROVT \\
\hline MKPQRO & MKOPNPP & MKNSTSSS & MKQOQMQN & MKNOVQVU & MKNMPTUV & MKQVQNNP & MKNTQVVQ \\
\hline$-\mathrm{NK}$ & MKVUU & -MKVTU & NK & MKNTO & -MKNNU & MKMT & MKOSO \\
\hline MKNRSNVO & MKMPOMVVN & MKMNSOTS & MKMPVRPPP & MKMONNTMN & MKMNSTRMO & MKMSTNPMO & MKMPMNSNS \\
\hline MKMNPTMVN & MKMNVQSRP & MKMNMMMOQ & MKMMVNRNNV & MKMNMPOMO & MKMMUMSUVQ & MKMNQOSVO & MKMNOMNNO \\
\hline MK & MKNRQSMV & MKOMPVRV & MK & MKVSVSUV & MKVTSTON & MKVVRRPN & MKVROQRR \\
\hline $\mathrm{N}$ & 0 & $P$ & $\mathrm{Q}$ & $\mathrm{R}$ & $\mathrm{S}$ & $\mathrm{T}$ & $\mathrm{U}$ \\
\hline $\mathrm{s}$ & $\mathrm{s}$ & $\mathrm{s}$ & $\mathrm{s}$ & $\mathrm{s}$ & $\mathrm{s}$ & $\mathrm{S}$ & $\mathrm{s}$ \\
\hline MKPMPONQ & MKPNMMSR & MKNVRUOU & MKOPMSUS & MKOOUSOV & MKNVNQMN & MKUUNOTS & MKOQSUMV \\
\hline MKQRNUQP & MKQNPUVS & MKORRSUR & MKPNPPUQ & MKPPQVRT & MKORONRT & MKSVTQOV & MKPOMSUR \\
\hline MKUV & - MKOQ & -MKSVQ & -MKPQS & -MKNMU & -MKUNO & -MKVPO & -MKTMQ \\
\hline MKMTTRTUV & MKNMVONO & MKMPTSMMS & MKMRUSTUQ & MKMTVMSPQ & MKMPTVRNN & MKNQUP & MKMROQOMQ \\
\hline MKMNQUMT & MKMOPQOUN & MKMNOTMST & MKMMUQUOT & MKMNQVMVR & MKMNMVMSP & MKMNNORUP & MKMNRNOMN \\
\hline MKQQMTUO & MKVSRRPU & MKTNSRNP & MKVPPUOQ & MKVURMOS & MKRTTPQN & MKPRTORR & MKTMUMUT \\
\hline $\mathrm{N}$ & 0 & $\mathrm{P}$ & $\mathrm{Q}$ & $\mathrm{R}$ & $\mathrm{s}$ & $\mathrm{T}$ & U \\
\hline $\mathrm{T}$ & $\mathrm{T}$ & $\mathrm{T}$ & $\mathrm{T}$ & $\mathrm{T}$ & $\mathrm{T}$ & $\mathrm{T}$ & $\mathrm{T}$ \\
\hline MKMMQVOTU & MKNPUPQV & MKMNPVTST & MKMMPRVNU & MKMNTNVSP & MKMNOSQMT & MKMMUQUNQS & MKMMTNTONU \\
\hline MKMMRUNSPV & MKNTSSOS & MKMNTQVOV & MKMMQPRSNN & MKMOONVQS & MKMNRSSMS & MKMNNMOV & MKMNONVPU \\
\hline -МКРТО & MKOUS & MKPMS & -MKQRU & MKSNS & MKSOQ & MKSMU & MKTSU \\
\hline MKMMNSNOVP & MKMORUVVO & MKMMPRTPNO & MKMMNMPMPS & MKMMOVMOVT & MKMMPOSQRR & MKMMORQSNV & MKMMOROMQV \\
\hline МКМмОМОМоU & MKMNTTSPQ & MKMMPRPVMU & MKMMNOTVRQ & MKMMQUMNMQ & MKMMPOPTMQ & MKMMOVPVVN & MKMMPRMVMP \\
\hline MKSVNTV & MKVRNUQV & MKVNPORQ & MKRRRTQS & MKTQSTSS & MKTQMMUN & MKTNOOUS & MKRRPUUS \\
\hline $\mathrm{N}$ & 0 & $P$ & $Q$ & $\mathrm{R}$ & S & $\mathrm{T}$ & $\mathrm{U}$ \\
\hline U & $\mathrm{U}$ & $\mathrm{U}$ & U & $\mathrm{U}$ & $\mathrm{U}$ & U & U \\
\hline MKPUOTNN & MKMQNQTNT & MKNONQUR & MKMROPUST & MKMNNPQOS & MKMNNTUSV & MKQTPTNP & MKMNTNSUU \\
\hline MKPTTQOS & MKMQRQOSN & MKMSUPSRQ & MKNMPOMQ & MKMOOPMPR & MKMOOQTRO & MKPSUNTN & MKMPQVTPO \\
\hline$-\mathrm{NK}$ & -MKUUU & -MKMUQ & -MKRPS & MKRV & MKPOQ & -MKTUU & MKTS \\
\hline MKMNSOPR & MKMNOMNVP & MKOTVUTP & MKMNQQPOP & MKMMQTNOST & MKMMQSUUT & MKOPVUU & MKMMSTRTMU \\
\hline MKMNPTMTO & MKMMVUOQMT & MKMMSUTTQT & MKMMRPNMOT & MKMMQRNOPV & MKMMPURQTU & MKMNPNSTR & MKMMRVRSPO \\
\hline MK & MKQQORQT & MKUOPRV & MKUONMMT & MKQVOPOS & MKSUVVN & MKSMVTRQ & MKQOTRNN \\
\hline $\mathrm{N}$ & 0 & $P$ & $Q$ & $\mathrm{R}$ & S & $\mathrm{T}$ & $\mathrm{U}$ \\
\hline $\mathrm{V}$ & $\mathrm{V}$ & $\mathrm{V}$ & $\mathrm{V}$ & $\mathrm{V}$ & $\mathrm{V}$ & $\mathrm{V}$ & $\mathrm{V}$ \\
\hline MKPRPMTP & MKPRRSRQ & MKPMQMON & MKNUTQV & MKQPVQPN & MKPUTNPQ & MKROPPTU & MKQMSVQN \\
\hline MKPTSMUR & MKPSTVP & MKPNOTVQ & MKOOQNST & MKQNRSUR & MKQMPVVT & MKRQOQV & MKPVTPRP \\
\hline MKQQU & -MKPQQ & MKVSS & MKNNO & -MKRTU & -MKTMQ & MKQTQ & $-\mathrm{MKRV}$ \\
\hline MKNUTMPS & MKNPPVPS & MKNQPPVV & MKMUQVVQ & MKNUQOQR & MKNNQN & MKNUUOQU & MKNSQRQU \\
\hline MKMNPPUUR & MKMOONNVR & MKMNOSQST & МКMMTPTPOT & MKMNRPQQP & MKMNOUNNN & MKMNPTVNO & MKMNRVMSU \\
\hline MKUVOSUT & MKVPTTTR & MKONNVTS & MKVVMSSS & MKUNRSOS & MKTNMVNN & MKUTTVV & MKUMSVTN \\
\hline $\mathrm{N}$ & 0 & $P$ & $\mathrm{Q}$ & $\mathrm{R}$ & $\mathrm{s}$ & $\mathrm{T}$ & $\mathrm{U}$ \\
\hline NM & NM & NM & NM & NM & NM & NM & NM \\
\hline MKMTNPSOU & MKNTMVMV & MKNRPTTT & MKMTTQSU & MKNNTPQQ & MKNNUSSU & MKPVTQPS & MKOOSQPS \\
\hline MKNNUVRT & MKOMTSUT & MKOPOSVS & MKNMNONP & MKNTNUTV & MKNRQPTN & MKQQNSPR & MKOTMUOR \\
\hline MKURU & -MKUTS & MKSVU & MKVV & MKORQ & -MKQT & -MKVTU & -MKUNO \\
\hline MKMONNMSR & MKMOTSPQU & MKMQSRNQT & MKMNQSTOT & MKMPTSTMN & MKMOVVUOO & MKMPMTMOS & MKMOVVNPU \\
\hline MKMMVNNPUV & MKMNUUMVQ & MKMNONURN & MKMMRUURN & MKMNNUUOO & MKMMVQSUUO & MKMNPUOSR & MKMNQMSTP \\
\hline MKQVOOSN & MKQUOTVQ & MKTMNOT & MKNNUMNS & MKVRMQNS & MKUTPOUQ & MKOMPVRV & MKRUMUMO \\
\hline $\mathrm{N}$ & 0 & $P$ & $Q$ & $\mathrm{R}$ & S & $\mathrm{T}$ & $\mathrm{U}$ \\
\hline NN & NN & NN & NN & NN & NN & NN & NN \\
\hline MKMPOUVVR & MKNVRSMR & MKMNVQSQ & MKMOTSMVV & MKMVNPOUN & MKMRQRUVS & MKMQOUSPQ & MKMQRUQRQ \\
\hline MKMPRMOP & MKOOSOQP & MKMOTQRUP & MKMPQQQQQQ & MKMUSOQV & MKMSMQOUO & MKMQTPPOP & MKMRQVOPT \\
\hline$-\mathrm{NK}$ & -MKRRS & -MKNV & -MKQMQ & -MKSOO & -MKUVS & -MKSUO & -MKTQQ \\
\hline MKMMRRVTQS & MKMRQTPVU & MKMMSTNVUU & MKMNMOVNP & MKNMRRVO & MKMNQSQQN & MKMNMVTQQ & MKMNNQQTS \\
\hline MKMMRNUPVP & MKMOMSPUN & MKMMQUOMNV & MKMMPPQPS & MKMMURNS & MKMMRVPQTT & MKMMRUQSTS & MKMMTOPPQP \\
\hline MK & MKUOOPOQ & MKTSMVRV & MKUNNOPQ & MKTRPUOR & MKQPRPUU & MKSUUPQU & MKSPNUTO \\
\hline $\mathrm{N}$ & 0 & $P$ & $Q$ & $\mathrm{R}$ & S & $\mathrm{T}$ & $\mathrm{U}$ \\
\hline NO & NO & NO & No & NO & NO & NO & NO \\
\hline MKPPVNMV & MKPNVTQN & MKPSNRUR & MKNQNQP & MKOOVSNU & MKOMNTUQ & MKPUPONR & MKNTONPV \\
\hline MKPRMURO & MKPSNONT & MKPVRQSU & MKNUNNMN & MKOTVPUN & MKOQPMSU & MKQPRQMP & MKONMMSO \\
\hline$-M K Q Q$ & -MKQV & -MKRPO & MKQSU & MKRUU & -MKQMQ & MKRPS & MKPPU \\
\hline MKNPRSQR & MKMUMOVOV & MKMUNQOTN & MKMPRVTUT & MKMSORRMP & MKMPTMUU & MKNQRVTU & MKMSQTRST \\
\hline MKMNPTNSR & MKMOPSRST & MKMNPVSON & MKMMSTRNRP & MKMNPROTU & MKMNNORVU & MKMNPTNMS & MKMNPSSMO \\
\hline MKUVUUVT & MKUTNQQS & MKUQTRVO & MKUTRRMR & MKUMTNVO & MKVNPQUQ & MKUPTVNV & MKVPUUVO \\
\hline $\mathrm{N}$ & 0 & $P$ & $Q$ & $\mathrm{R}$ & S & $\mathrm{T}$ & $\mathrm{U}$ \\
\hline NP & NP & NP & NP & NP & NP & $\mathrm{NP}$ & NP \\
\hline MKQTSRTT & MKRQRVR & MKPSMRQU & MKOTQOOR & MKPSRUON & MKPVPPST & MKTMTQPV & MKPSQROQ \\
\hline MKRTPOO & MKSMUUQP & MKQSRMVP & MKPUOSRU & MKQURORT & MKRNRRMQ & MKTNUVPV & MKQUUUOV \\
\hline MKV & MKTUQ & MKVOU & MKTR & MKOUU & MKNUS & -MKPQQ & MKNSQ \\
\hline MKNPOTUQ & MKNNVUVR & MKMTUMPUP & MKMQTORQT & MKMROQTUN & MKMRVNPVS & MKNTOPSN & MKMRQUMSR \\
\hline $\begin{array}{c}\text { MKMNPUSSU } \\
\text { MKกPNTMYV }\end{array}$ & $\begin{array}{c}\text { MKMONTQPV } \\
\text { MKSONPIIP }\end{array}$ & $\begin{array}{l}\text { MKMNPRVRO } \\
\text { MKPSTRON }\end{array}$ & $\begin{array}{l}\text { MKMMUVMUMR } \\
\text { MKSRTROก }\end{array}$ & $\begin{array}{c}\text { MKMNRPRTS } \\
\text { MKVRONVT }\end{array}$ & $\begin{array}{c}\text { MKMNOSNNR } \\
\text { MKVIIMOTP }\end{array}$ & $\begin{array}{c}\text { MKMNMQMOT } \\
\text { MKVPRSPS }\end{array}$ & $\begin{array}{c}\text { MKMNSQOOP } \\
\text { MKVIIPPIIN }\end{array}$ \\
\hline
\end{tabular}




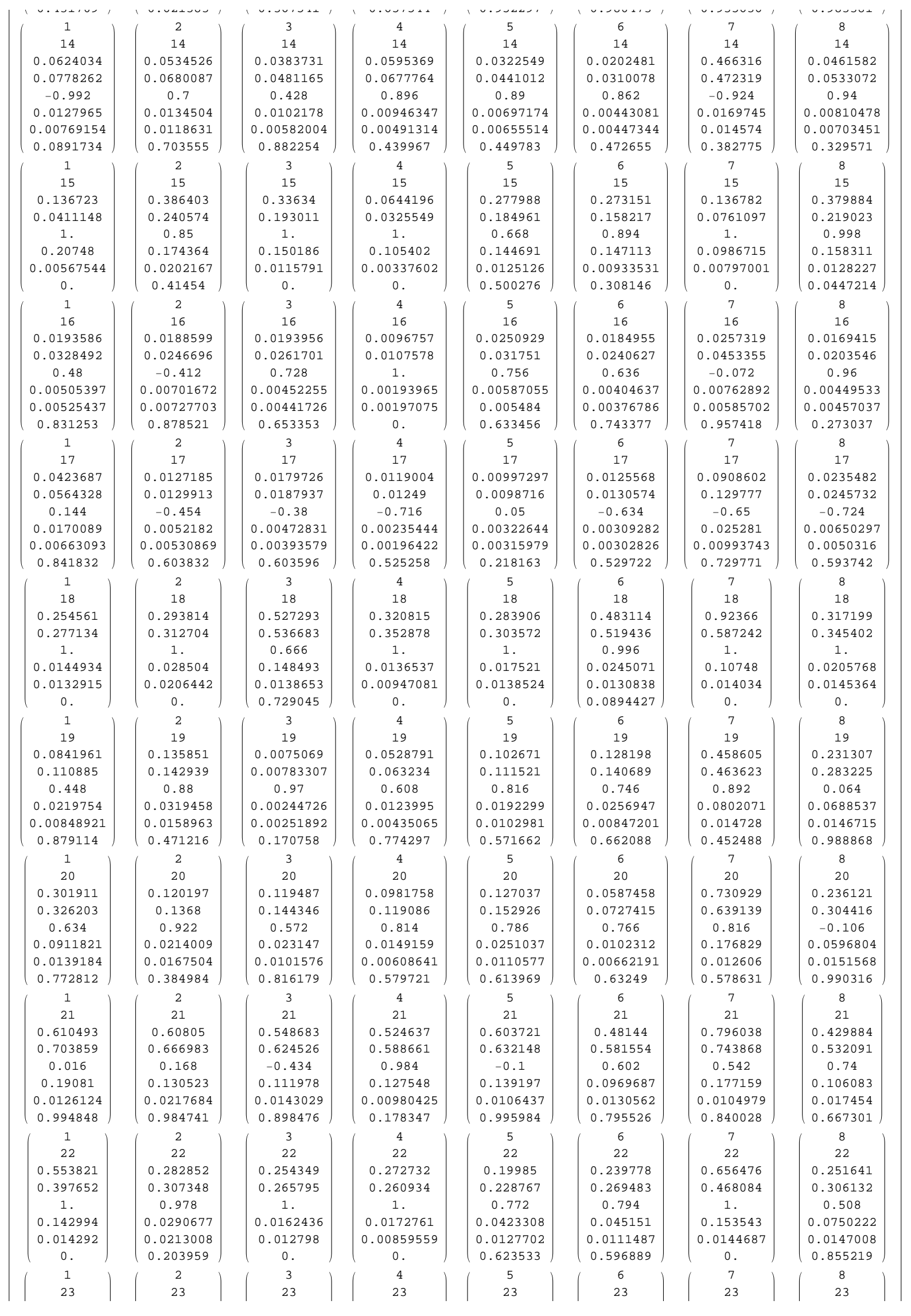




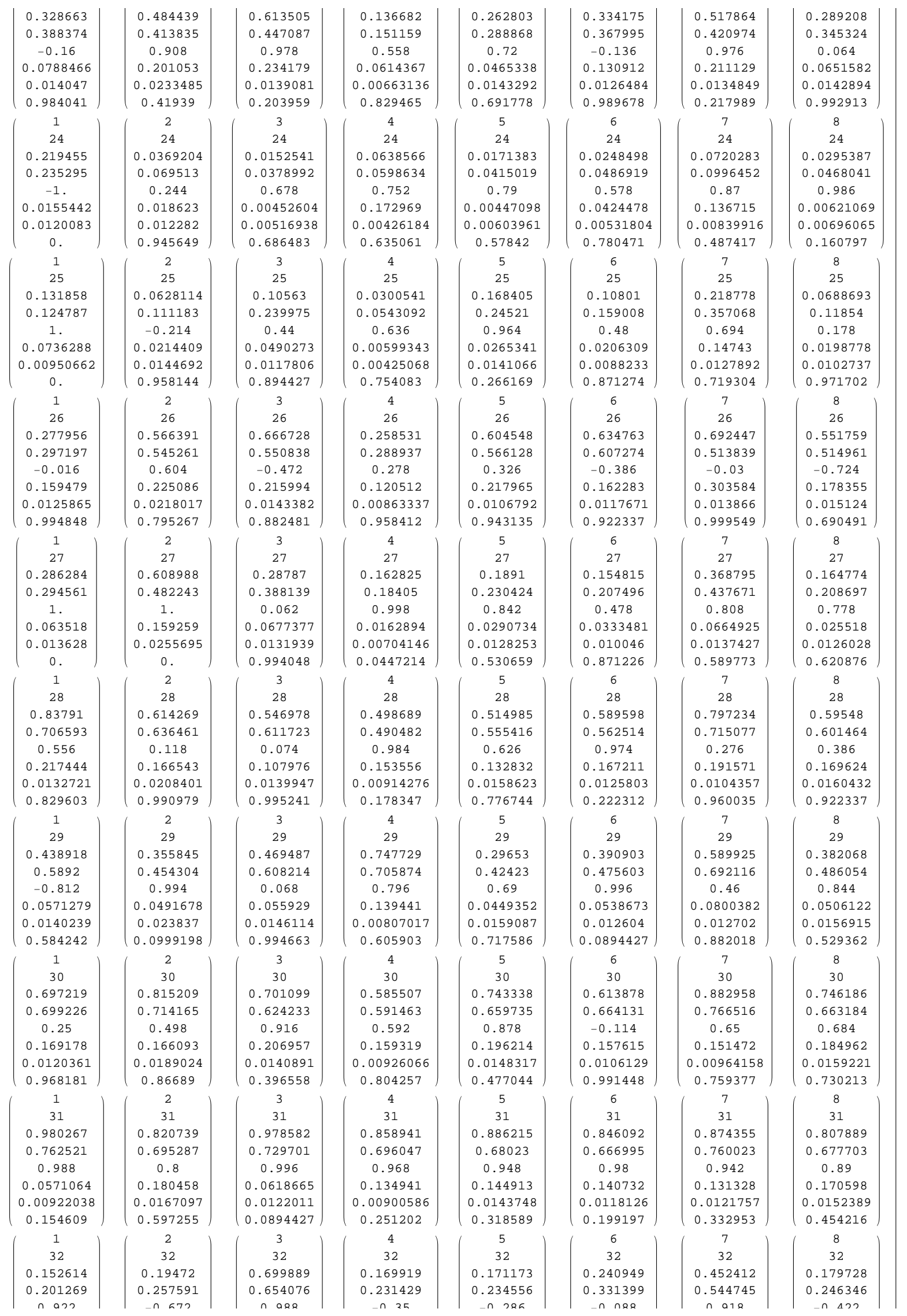




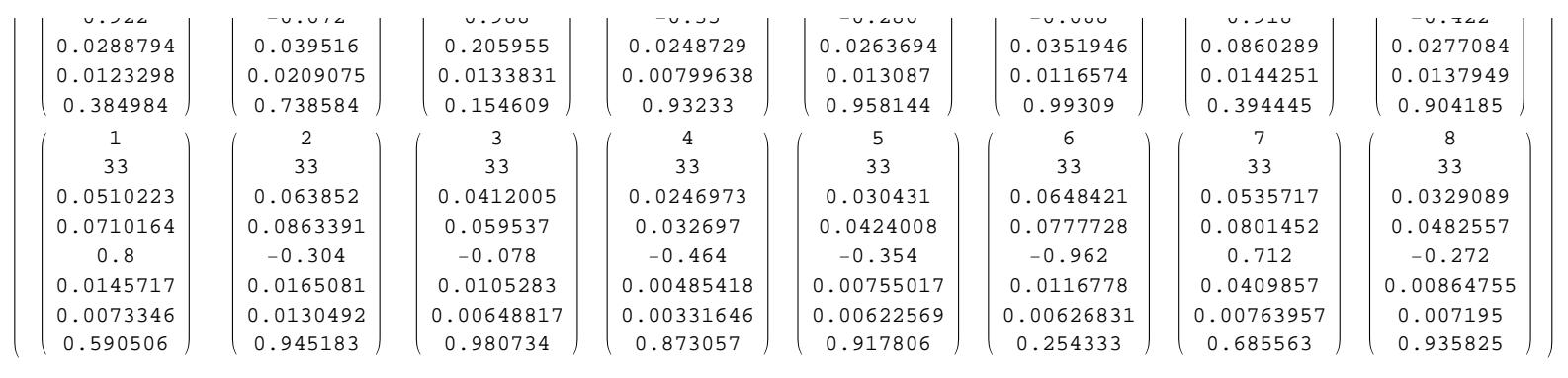

We conservatively weight the reduction by mean \pm

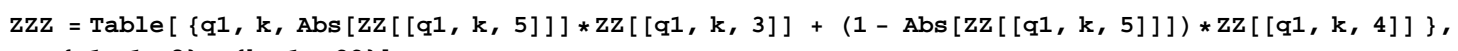
\{ènI NI U\} I \{âI NI PP\}]X

\section{j 1̇êáñcçêã [qê åëéçëÉ [www] ]}

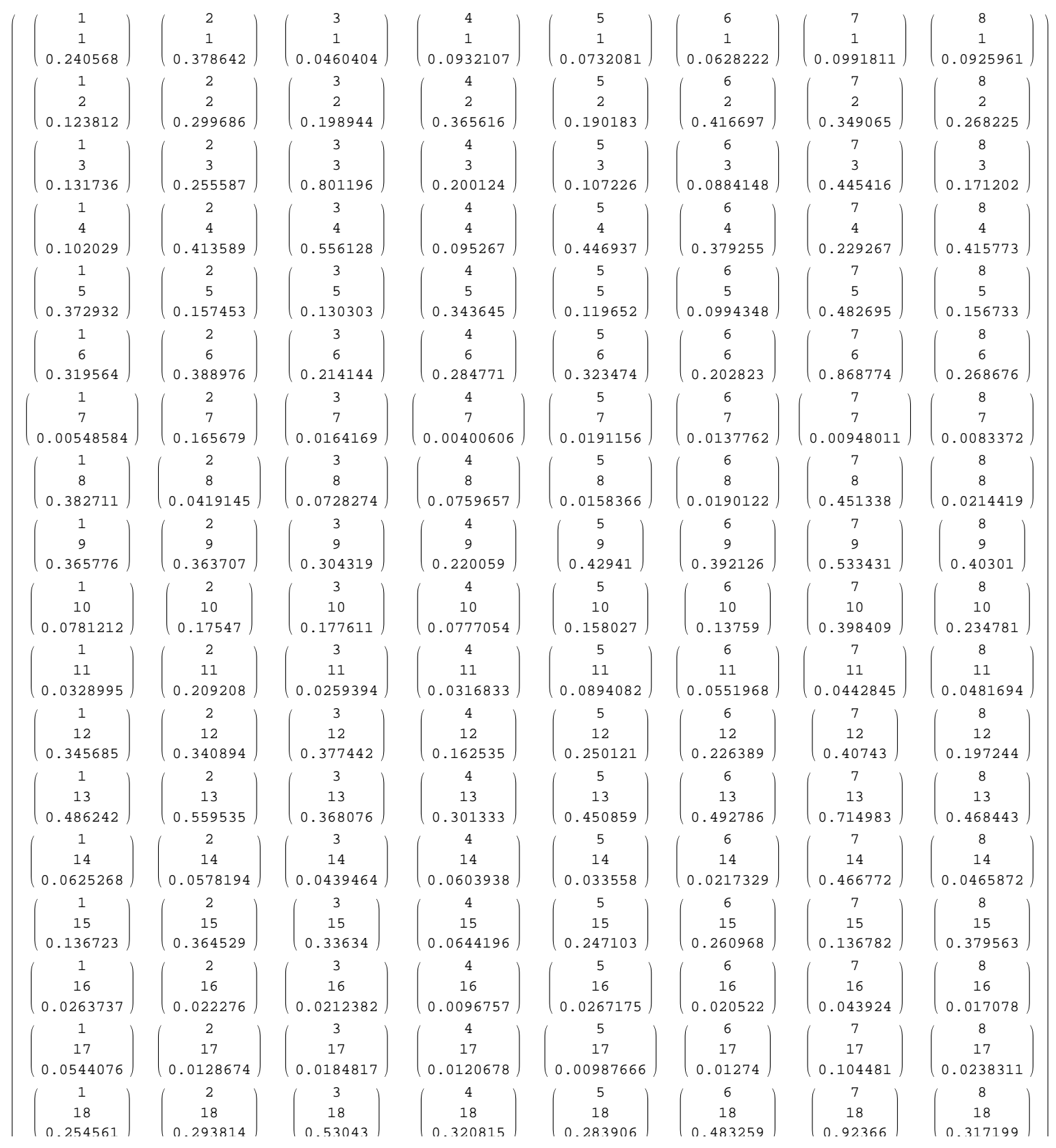




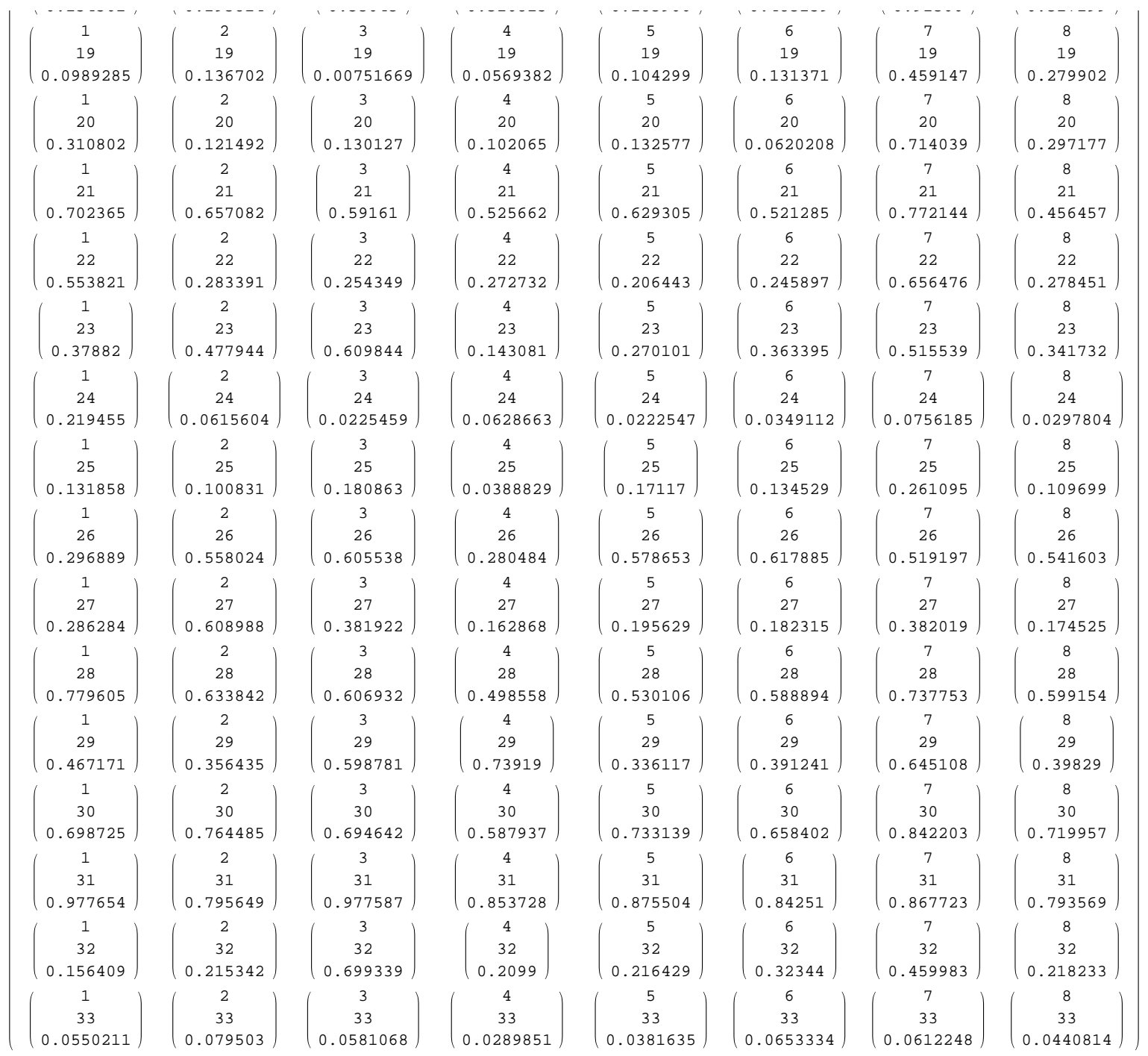

This has SD

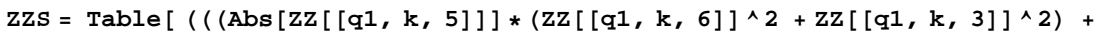
$(N-\wedge \ddot{A} \ddot{e}[w w[[e ̀ N I$ âI R] $]]) *(w w[[e ̀ N I$ âI T] $]\{0+w w[[e ̀ N I$ âI Q] $]\{0)-$

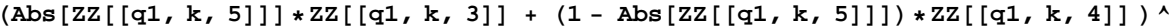
O)) $\{(K R))$ I $\{$ èNI NI U $\}$ I $\{\hat{I}$ NI PP $\}$ ]
} 


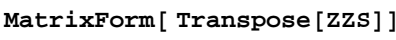

\begin{tabular}{|c|c|c|c|c|c|c|c|}
\hline IVV & DQQT & RMTUR & MOS & TV & $\mathrm{PN}$ & $\mathrm{MI}$ & NTTOP \\
\hline МКMРМTTTO & MKMROQOSR & MKMQQQPV & IKMNMMQPV & MKMPUUNNR & MKMPROPSR & MKMRSQMPT & MKMPPOQQT \\
\hline MKMRPQSUP & MKOTSMU & MKNTVRPR & JOQQ & MKMPSUMRS & IKMROPSMT & STR & NSRO \\
\hline MKMMVUQOUO & MKMVTTTMS & MKOMNMPP & IKMMRURROS & MKMOMUMSN & IKMOTOMRO & MKMN & VSUN \\
\hline MKNRSNVO & MKMPOVVTP & MKMNTNMU & MKMPVRPPP & MKMORNPUO & IKMNROPUQ & MKMQTOVRR & MKMPRUPPU \\
\hline MKMUSURNT & DQPMN & MKMQOPORU & UON & $S$ & JVTN & $P$ & IV \\
\hline OTRQ & SQN & VMNVN & OPO & MF & UT & $\mathrm{T}$ & VS \\
\hline MKMI & RVS & OTMQU & MKM & TPO & RPN & $R$ & NS \\
\hline MKNOSNMP & MKN & MVSV & MKMP & MKNQMVNU & SOVST & MKNPMPPV & UUV \\
\hline MKMORUUQR & MKMOVPOO & MKMRPRRP & MKMNQUMMU & MKMPOMUOU & MKMOUMSPO & MKMPNNNRN & MKMPOSOUO \\
\hline MKMMRRVTQS & MKMQRSUOP & MKMMSNMOMS & MKMMTTVMVR & MKMUPQTUO & MKMNQNMSO & MKMMVUSSNP & MKMNNORNP \\
\hline MTQTS & MKMS & OQURQ & STP & MKMRQRRNT & rQS & $\mathrm{P}$ & $\mathrm{JN}$ \\
\hline 7PPT & R & $\mathrm{P}$ & PS & $\mathrm{V}$ & RNOPO & $\mathrm{V}$ & VP \\
\hline MKM & MKM & $2 \mathrm{~V}$ & JOO & MKMM & JQNQ & Vo & JTV \\
\hline MKOMTQU & MKN & MKNRMNUS & MKNMRQMO & MKNOSPNU & MKNQPRRV & MKMY & MKN \\
\hline MKMMUQUTUP & IKMМTTOMMO & MKMMRQNNRU & MKMMNVPVSR & MKMMSQQTQR & MKMMQTTMPO & MKMMTURRMV & MKMMQRQTUN \\
\hline MKMNMNUOP & MKMMR & MKMMQOTP & MKMMOOSSNS & MKMMI & MKMMPMTUUN & MKMO & MKMN \\
\hline MKMNQQVPQ & MKN & MKNONROV & MKMN & $\mathrm{ON}$ & RTVN & QU & ISU \\
\hline MKMOMTVNP & MKMPI & QRMMT & M & V & $\mathrm{QT}$ & MKMTRVOOS & $\mathrm{V}$ \\
\hline MKM' & QMP & OOQMPR & MKMN & UQN & MKMNNOMRN & S & $\mathrm{J} Q \mathrm{R}$ \\
\hline MKMO & MO & NN & JR & $\mathrm{TQ}$ & $\mathrm{RR}$ & $\mathrm{NU}$ & so \\
\hline MKNQOVVQ & MKMO & MKMNSOQPS & MKMNTOTSN & MKMPVRVQP & MKMQOOVNT & MKNF & MKMSMUVMP \\
\hline MKMQMQVOP & MKNVOTVR & MKOPOUUN & MKMQSSSNP & MKMQNUTRP & MKMRNMOPR & MKOM & MKMORROQ \\
\hline MKMNRRQQO & MKMN & MKMNNRVRN & MKNRMMO & MKMNNMQON & MKMPQRORV & VP & MKMMS \\
\hline MKMTPSOUU & MKMO & MKMTQTNRV & IKMNOUSVP & UQOV & MKMO & QV & MKMOOTSNR \\
\hline MKM & $P$ & RVSNO & $P$ & 0 & & $\mathrm{P}$ & $R$ \\
\hline MKMSPRNU & RV & MKMPONPNV & MKMN & MKM & MKMF & MKMS & MKM \\
\hline MKNTQVVT & UTN & MKMPSQUTT & KNROPP & QN & KNSRMVO & MKNM & MKNMSNTP \\
\hline MKMTUPOQQ & MKMQ & MKMQMPVMN & MKNORRVV & MKMT & MKMRQMPMR & MKMT & MKM \\
\hline MKMUROPPQ & MKNOUPPR & MKNVVOSN & MKNOOTS & MKNURVRP & MKMRSQROS & MKNP & MKN \\
\hline MKMSNROPS & MKN & MKMSPTNQQ & MKNP & UPRU & SR & MKN & MKI \\
\hline MKMPML & MKM & MKOMQTUO & MKMPPQQUU & MKMI & MKMC & MKML & MKN \\
\hline MKMNF & $M$ & KMMUQS & PN & 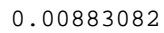 & 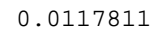 & SO & \\
\hline
\end{tabular}

To computes times for each marker need mutation rates 
j íêáñcçêã [ $\mu \mathrm{MM}]$

$\left(\begin{array}{cc}\text { MKMMMTOOOTN } & \text { MKNPTUUO } \\ \text { MKMMORONVO } & \text { MKNTRSMS } \\ \text { MKMMNPMPRT } & \text { MKRNSQTN } \\ \text { MKMMQVTTNR } & \text { MKNVVMRN } \\ \text { MKMMNOSUNO } & \text { MKNORRN } \\ \text { MKMMPNORUN } & \text { MKPPVQ } \\ \text { MKMMMMSQTPSS } & \text { MKOQQNRQ } \\ \text { MKMMMONROV } & \text { MKONRTUQ } \\ \text { MKMMPTSMNN } & \text { MKNNPNTR } \\ \text { MKMMNVPSVR } & \text { MKNMOMOO } \\ \text { MKMMMPSOOPN } & \text { MKOTPMTQ } \\ \text { MKMMOVRVTS } & \text { MKNNMMOV } \\ \text { MKMMTVVOOQ } & \text { MKMUQUUQT } \\ \text { MKMMMPVUNMR } & \text { MKNTSSV } \\ \text { MKMMOVTVTN } & \text { MKQSURUT } \\ \text { MKMMMNSPOSP } & \text { MKOMROSU } \\ \text { MKMMMNNQPTR } & \text { MKONRQP } \\ \text { MKMMPUMPSR } & \text { MKNRNRPO } \\ \text { MKMMMVVROVQ } & \text { MKNTVUVP } \\ \text { MKMMNNSPVR } & \text { MKONQNVS } \\ \text { MKMNNTMSP } & \text { MKNPVNPT } \\ \text { MKMMOSPPSV } & \text { MKNPPSNV } \\ \text { MKMMPVPNRT } & \text { MKMVVSVUR } \\ \text { MKMMMPOMRV } & \text { MKOPPNSS } \\ \text { MKMMNQMPVP } & \text { MKNTRTS } \\ \text { MKMMUNMPPT } & \text { MKOOUONT } \\ \text { MKMMONQRPV } & \text { MKNPMQRO } \\ \text { MKMNMSRSU } & \text { MKNNNPP } \\ \text { MKMMQSMOUO } & \text { MKOMQURU } \\ \text { MKMNQTN } & \text { MKMVQUPOQ } \\ \text { MKMNPOMON } & \text { OKMMQTO } \\ \text { MKMMOVMOU } & \text { MKNNOMUU } \\ \text { MKMMMOPPMUN } & \text { MKNQNMTS }\end{array}\right)$

Also need hyperbolic Bessel functions

$$
\begin{aligned}
& a ̊ a ̊=\operatorname{SMX~} \tau=\operatorname{KMNX}
\end{aligned}
$$

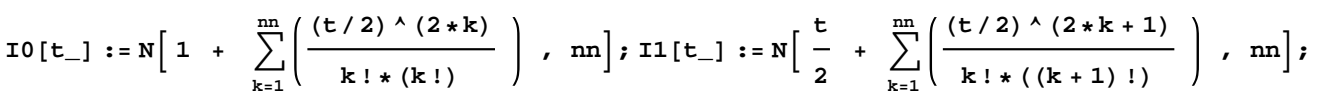

$$
\begin{aligned}
& \text { fO[ } 1 \mid] W=k\left[\frac{1\{0}{U}+\sum_{\hat{a}=\mathbb{N}}^{a ̊ a ̊ a ~}\left(\frac{(1 / 0)\{(O * \hat{a}+0)}{\hat{a} ! *((\hat{a}+0) !)}\right) I \text { åa }\right] x b b[1 \mid] W=k\left[N+\sum_{\hat{a}=N}^{a ̊ a ̊ a ~}\left(\frac{1\{\hat{a}}{a ̂}\right) I \text { åa }\right] x
\end{aligned}
$$

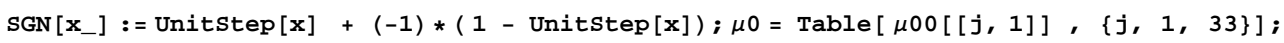

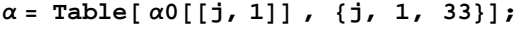

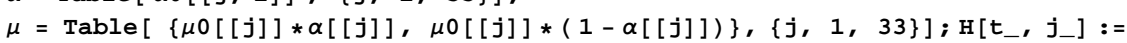

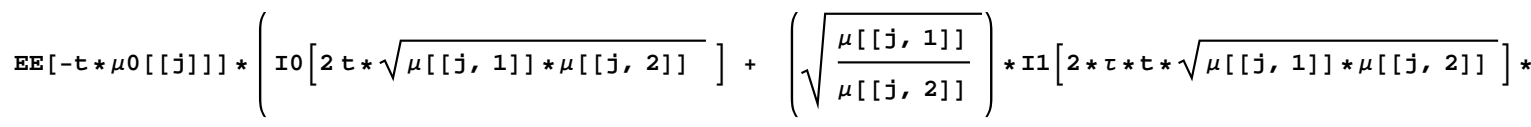

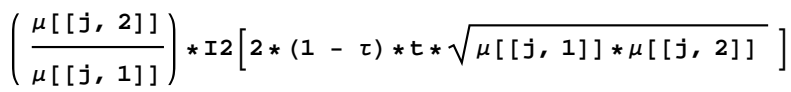

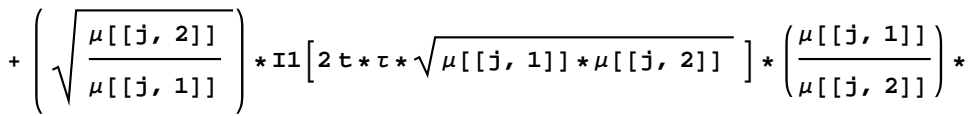

$$
\begin{aligned}
& \mathrm{fo}[\mathrm{O} *(\mathrm{~N}-\tau) * \hat{1} * \sqrt{\mu[\text { [àI N] }] * \mu[\text { [àI O] }]}]) \mathrm{x}
\end{aligned}
$$

The times $T$ for each marker are obtained by 
qp [â|] W= kçêã ä[fåîÉêëÉṕêêáËë[pÉêáÉë[ (N - e[ñI â])I \{ñI MI SM\}] ]]X qpp[í|I â|] W= qp[ â] /K ñ $\rightarrow$ IX

which is applied to give expansion times for each marker

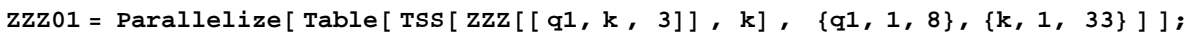

j íêáñcçêã [ qê åëéçëÉ [WwwMN] ]

QMTKOMP TQRKUUU SRKVQTS NPUKRN NMTKMTU VNKNQUO NQUKMVO NPTKROV

ROKORRP NRSKQVO VPKQNUU OMSKVPS UUKRRP ORPKMQP NVPKQOO NPRKOPO

NMUKTSR OOUKNVP NOVRKSR NTOKPMS UTKOSTN TNKNUQU QRVKONS NQOKTSN

ONKSUUP NMUKVMN NSTKN OMKNTON NONKMRR VTKNOUO ROKTNNR NMVKSTQ

QNPKMOT NQMKPRU NNPKRPO PSTKUOT NMPKPOR UQKOVRO SNVKONS NPVKSPN

NPNKMR NTMKVQV UMKMONN NNPKMVQ NPPKNQT TRKNNQN NMOPKUS NMRKNUQ

URKMUPQ OVOTKNR ORSKSVR SOKMSQU OVVKOVR ONQKVU NOTKOSU NOVKRU

OPPTKOS NVVKRVP PRPKQQ PSVKPVP TQKOQRS UVKPMNP OVQMKRT NMMKURV

NPQKNUN NPPKNMV NMQKQSR SVKSR NTMKNMN NQUKPPO OQRKPVQ NRQKQSP

QOKRVVP NMPKNRP NMQKRVR QOKPRVT VNKRVTN TUKQTOS OVMKMVS NQRKNRT

VOKQUTU SRQKTUR TOKSPUT UVKMMUN ORVKSPQ NRTKNPU NORKOVP NPSKRUR

NRUKTRO NRRKTMR NTVKUV SOKPOUV NMPKVMQ VNKVPUO OMNKROV TTKVUQO

NMQKOP NPUKQS SSKMMOS QVKTTQT VNKNMUU NMSKUSN OUVKNQN VTKQNMQ

NSPKQSP NRMKSUV NNPKRMP NRTKSSR USKMVRO RRKPPVP NTNVKON NOMKRP

QVKPSOP NROKPST NPTKTSP OOKPRNT VRKPPQT NMNKRUQ OVKPUR NSMKOOR

NSQKRVS NPUKSNQ NPOKMRU RVKSSVS NSSKTUP NOTKRPV OTTKSQU NMRKUTR

QVMKNNU NNPKOUR NSPKON NMSKNVV USKUNPT NNOKNRS VSUKTMU ONNKMST

UMKQPMT VSKMMOQ OOOKUPU NMTKPVN VNKVSUP NVNKQRS NNMOKTS NMRKUPO

NMRKRTO NOVKRNQ TKRUROR RVKNUSQ NNNKSUT NOPKNTT SRNKVUV PPVKPMO

PQOKUTR NNPKUQN NOOKTQP VQKOQRP NORKOVN RRKSNV NQROKTR POPKRQ

NUPKMUU NQQKPOV NMTKSVP UOKTQMO NOSKTSV UNKPRPO OVQKVTO SPKRPMP

PPQKNP NPMKUOR NNQKTRN NOQKUPR UVKUOSR NNMKONQ QRSKTTP NOUKMPR

NPMKQST NUPKTQT OUNKUNV QMKNSUO UPKVTUQ NOPKOOR OMTKTV NNPKQQT

UMRKPTP OMMKOMN TNKPUT OMOKSPO TMKORNP NNNKOST OQUKPSS VOKTROV

NMPKMN TSKVURT NQSKTRQ OUKQOPO NPTKUOT NMRKPMU OOSKRVR UQKOVVV

QTKQTUS NOVKMVQ NRQKRQR QQKMUOT NPVKQQP NSOKOMQ NNNKVQV NONKRMR

NSUKPSN RRNKROU OQUKOMN URKTSTU NMRKUVT VTKRTTR OQUKOVN VOKTVVQ

PMQKNQP NPTKNRP NOOKQVQ UMKRVO VMKTNQT NNPKUTS OPMKTPU NNUKSTO

NSTKUPN NMVKNPP OUMKSOO RTRKOVT NMMKPPP NORKOOU PQPKVRO NOUKVPT

NPOKOOR NVOKMOP NOVKRSR UNKQSUV NRUKUMV NMVKOVR PROKVRS NQTKSQS

NPMNKNN NVUKOON NOVUKTO OUNKVTV POVKVST OSNKSPQ PNNKPPV NVSKMNP

SNKNVQR UVKMPTP SVMKVR USKPOUO UVKRUON NRMKNOU ORSKUNP VMKOUVO

NPOKONV NVQKSP NPVKVRT SUKPOVP VMKRTN NRUKOOO NQTKUNP NMRKMTO

The SD for $\mathrm{T}$ are

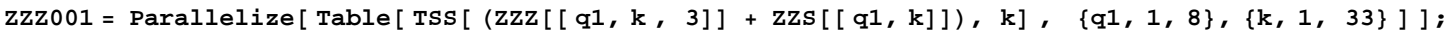

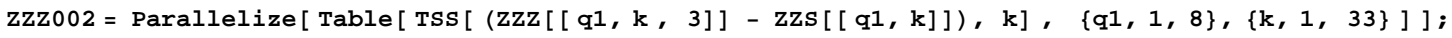
WWWMO $=$ MKR $*($ WWWMMN - WWWMMO $)$ 
j îêáñcçêã [ qê åëéçëé [wwwMo] ]



We are only using 29 markers

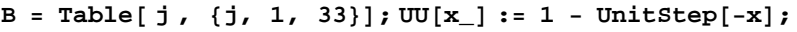

${ }_{-}=\operatorname{rr}[\{$ NI OI MI QI RI MI TI UI VI NMI NNI NOI NPI NQI MI NSI NTI

NUI NVI OMI ONI OOI OPI OQI ORI OSI OTI OUI OVI PMI MI POI PP\}]X_[à|] W= _[ [à]]X

We first sketch the spectrum of times

$n n[\tilde{n} \mid I$ èP $\mid I$ èQ $\mid] W=k\left[\frac{N}{\text { èQ } \sqrt{O * \pi}} *\right.$ bñé $\left.\left[-\frac{(\tilde{n}-\text { èP) \{O}}{O *(e ̀ Q)\{O}\right]\right] x$

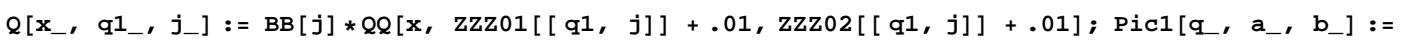

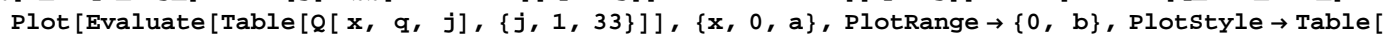

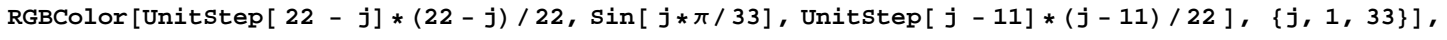
mäçíi $\ddot{A} E \ddot{E} \rightarrow$ \{è\} ]

This plots the time distributions for each marker 
máÅN [NI NMMMI MKN]

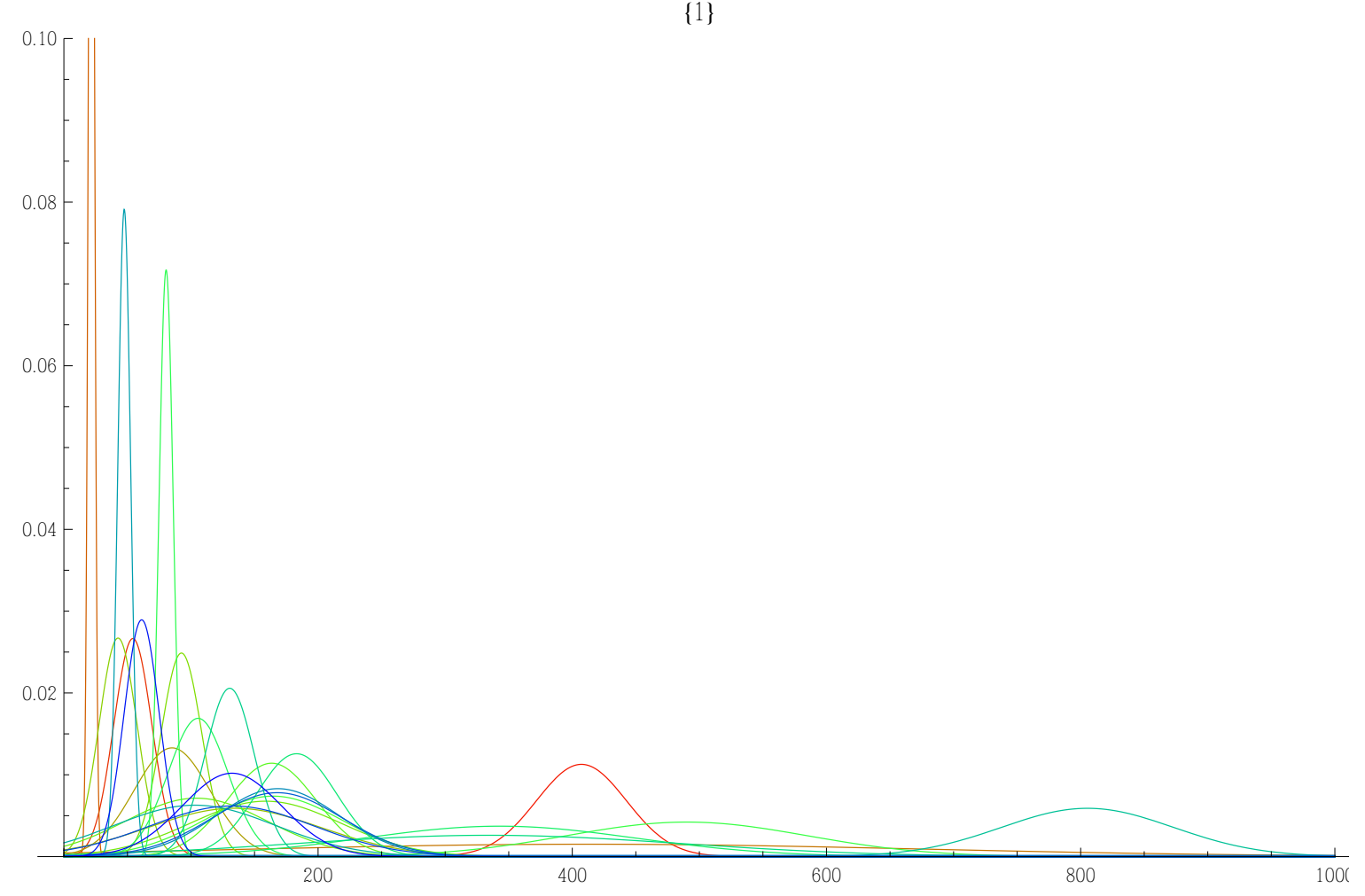


máÅN [OI NMMMI MKMQ]

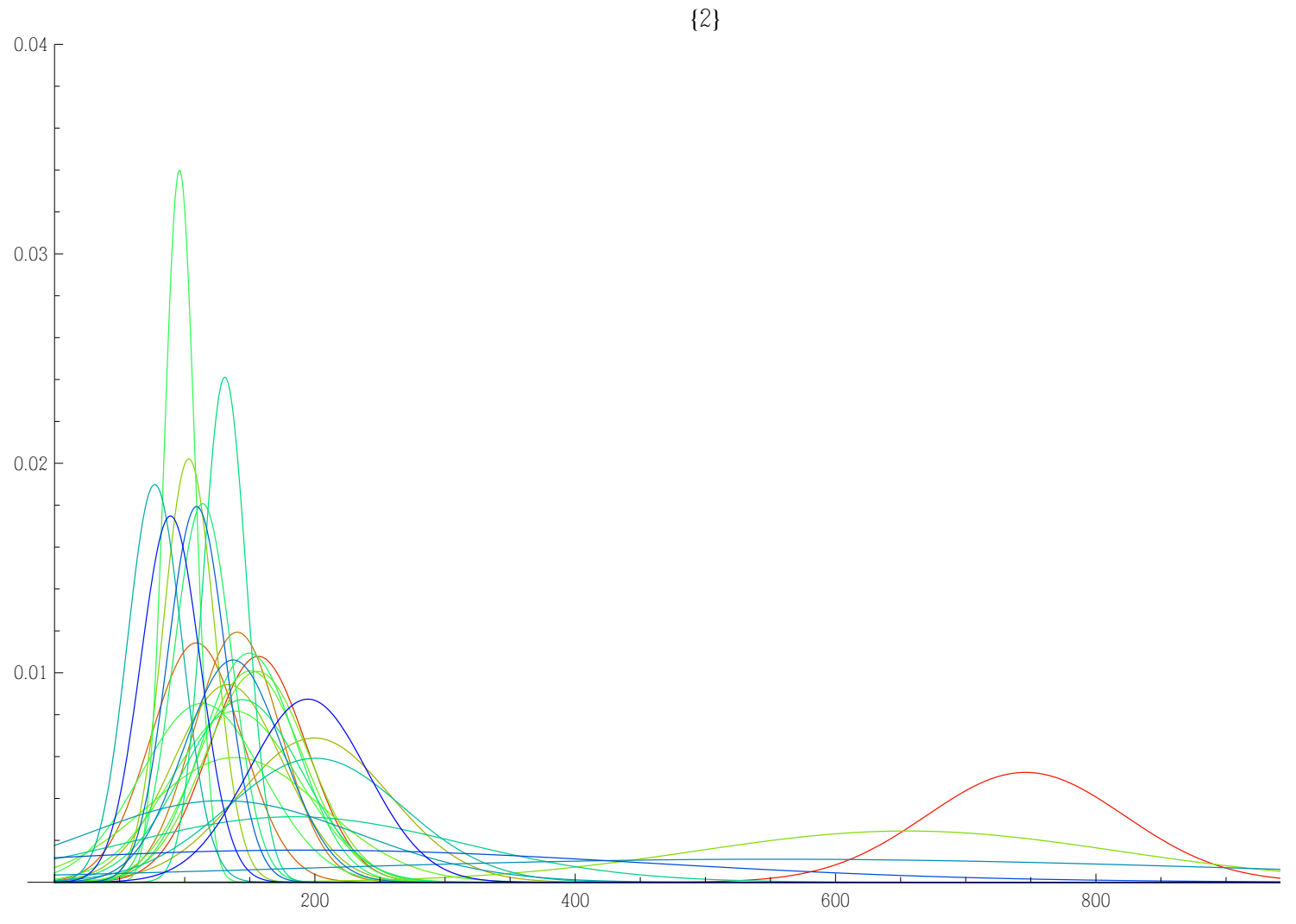


máÅN [PI SMMI MKMS ]

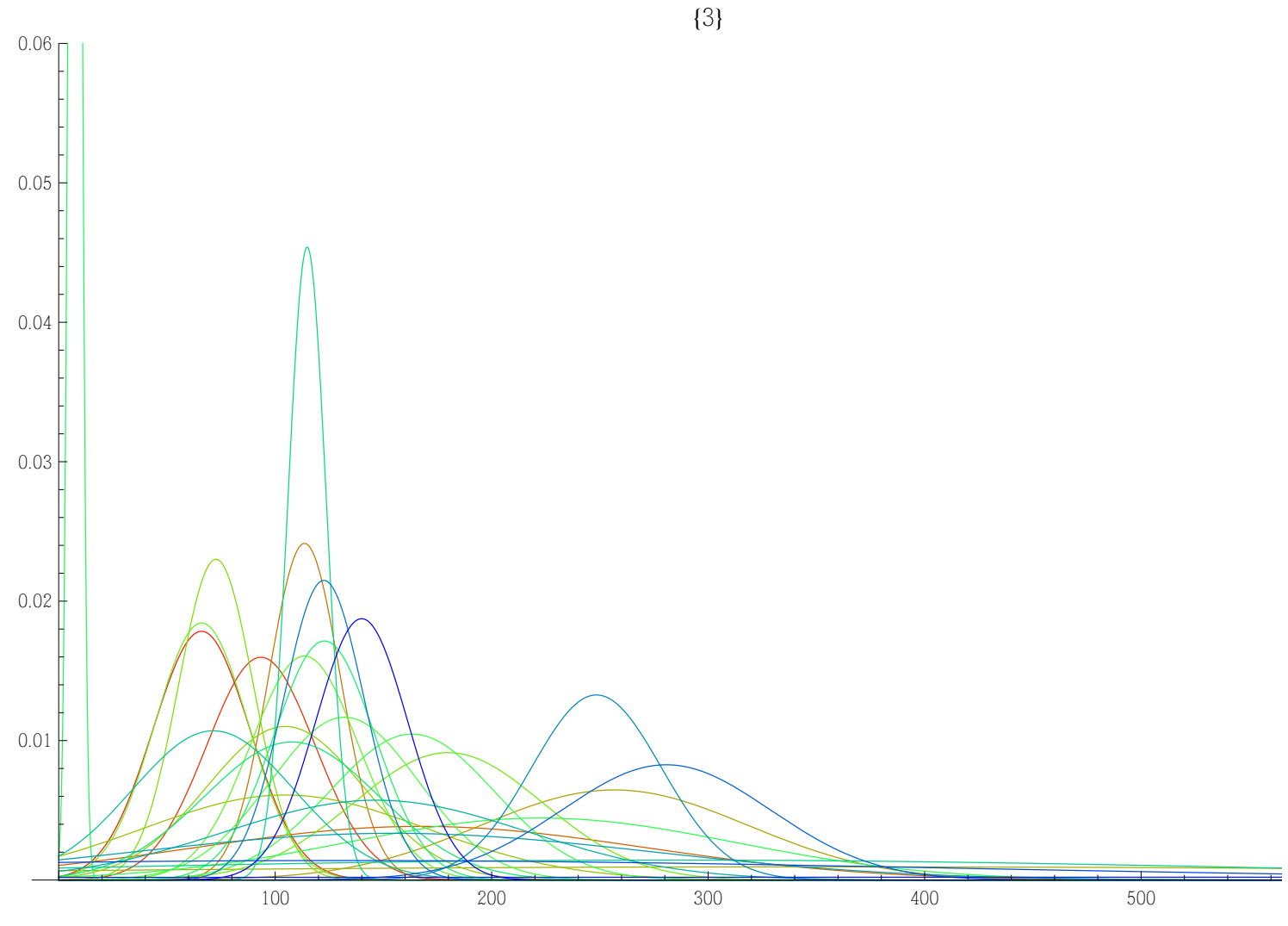


máÅN [QI SMMI MKN]

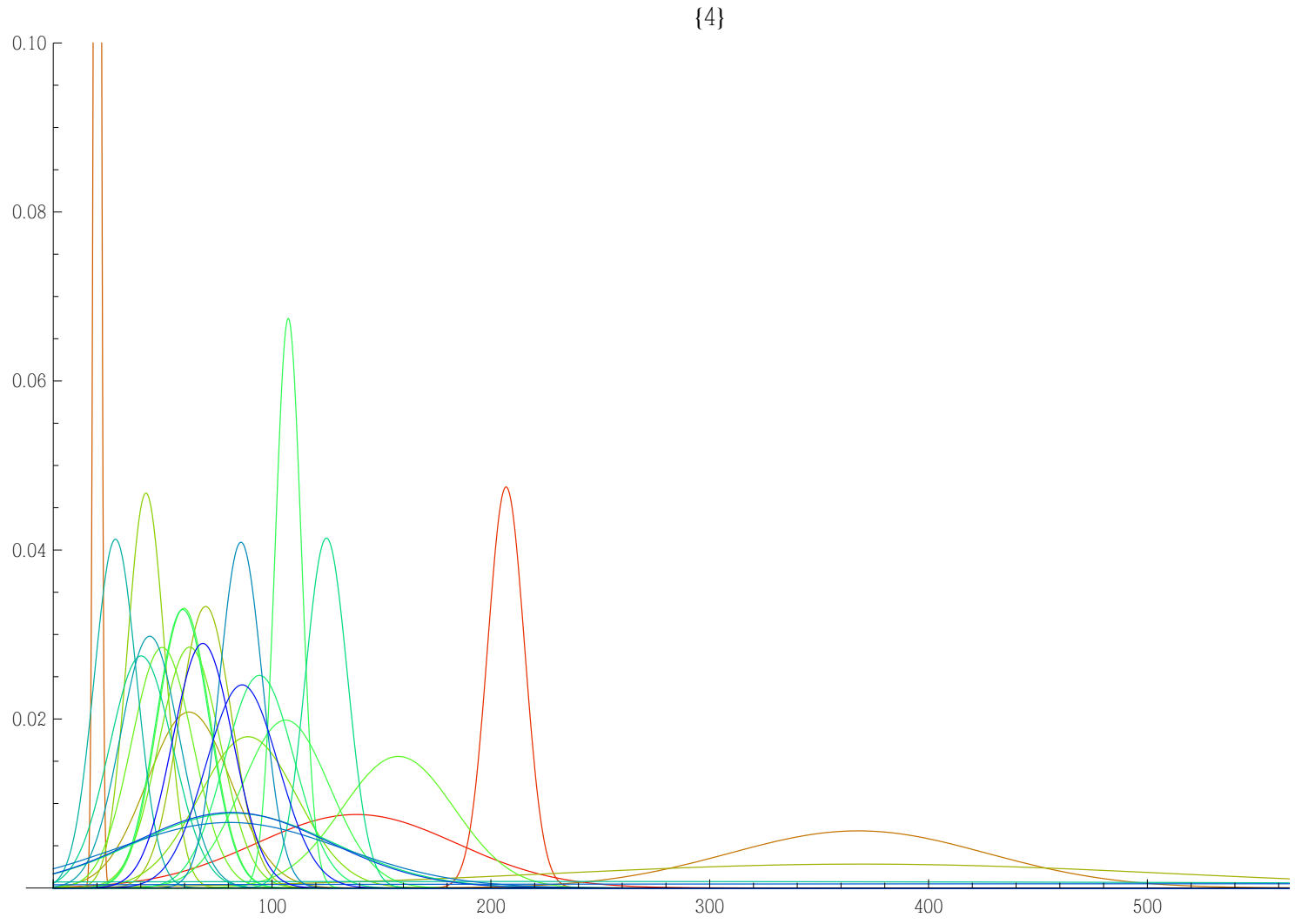


máÅN [RI SMMI MKMS ]

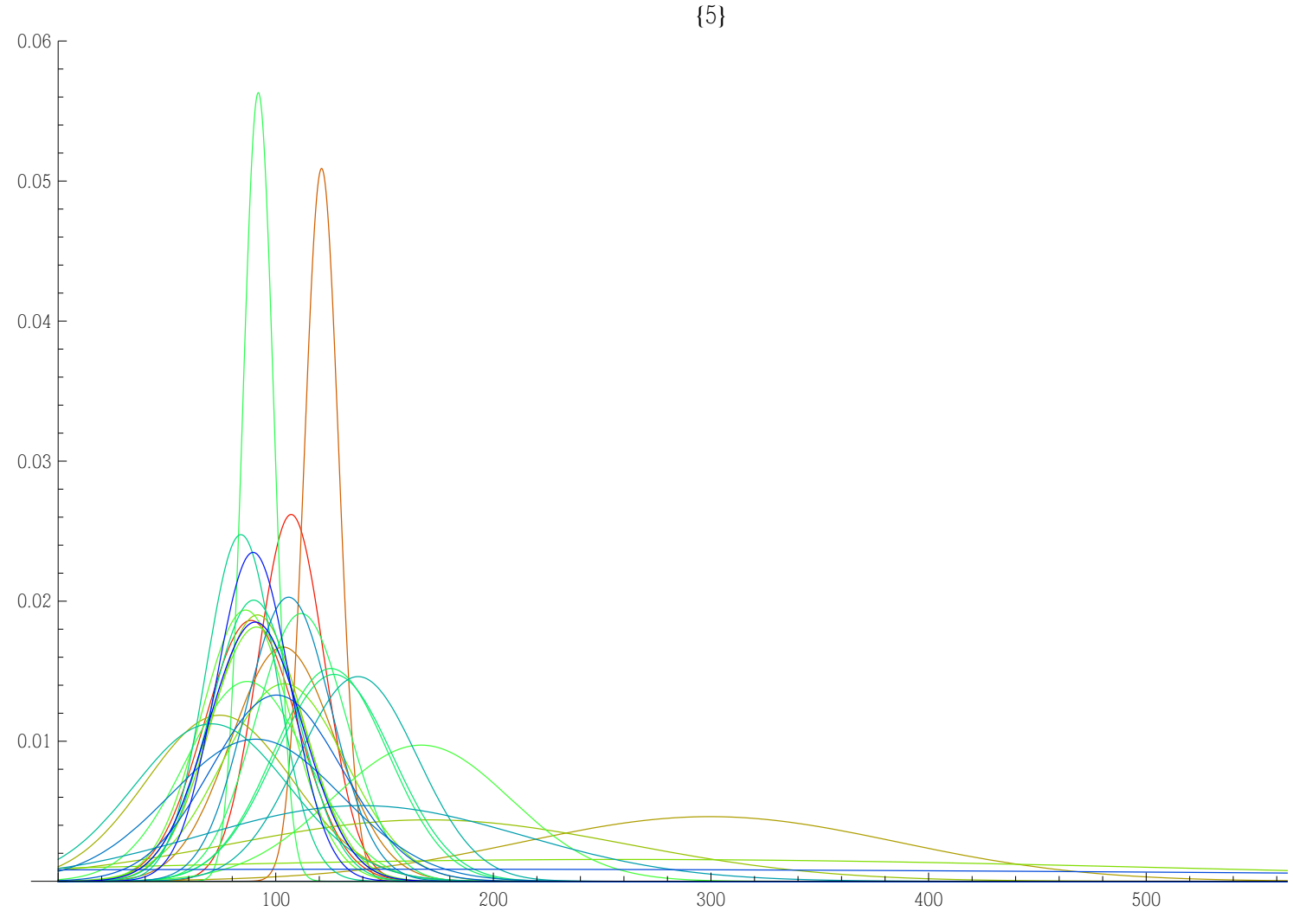


máÅn [SI SMMI MKMS]

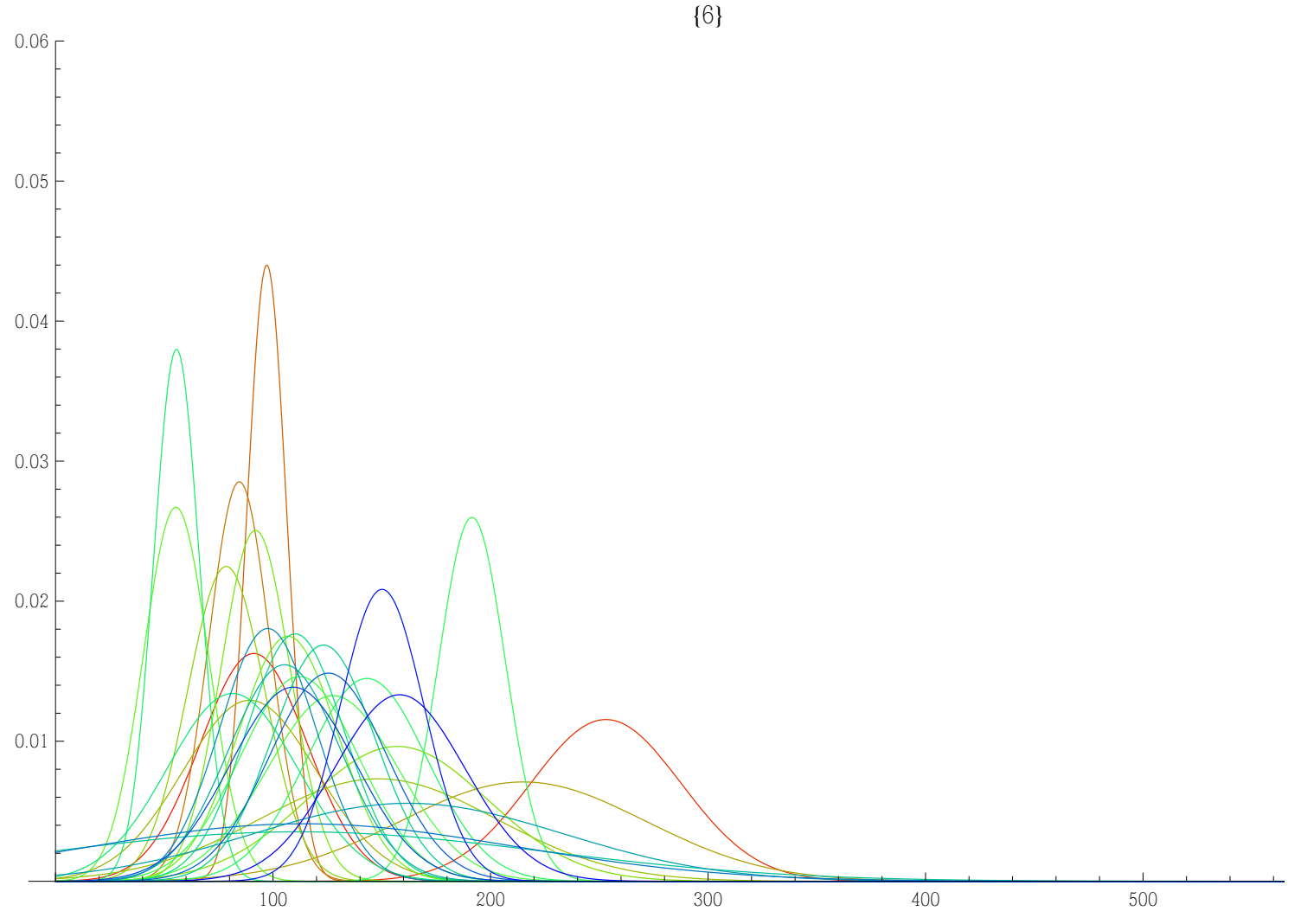


máÅN [TI NSMMI MKMP]

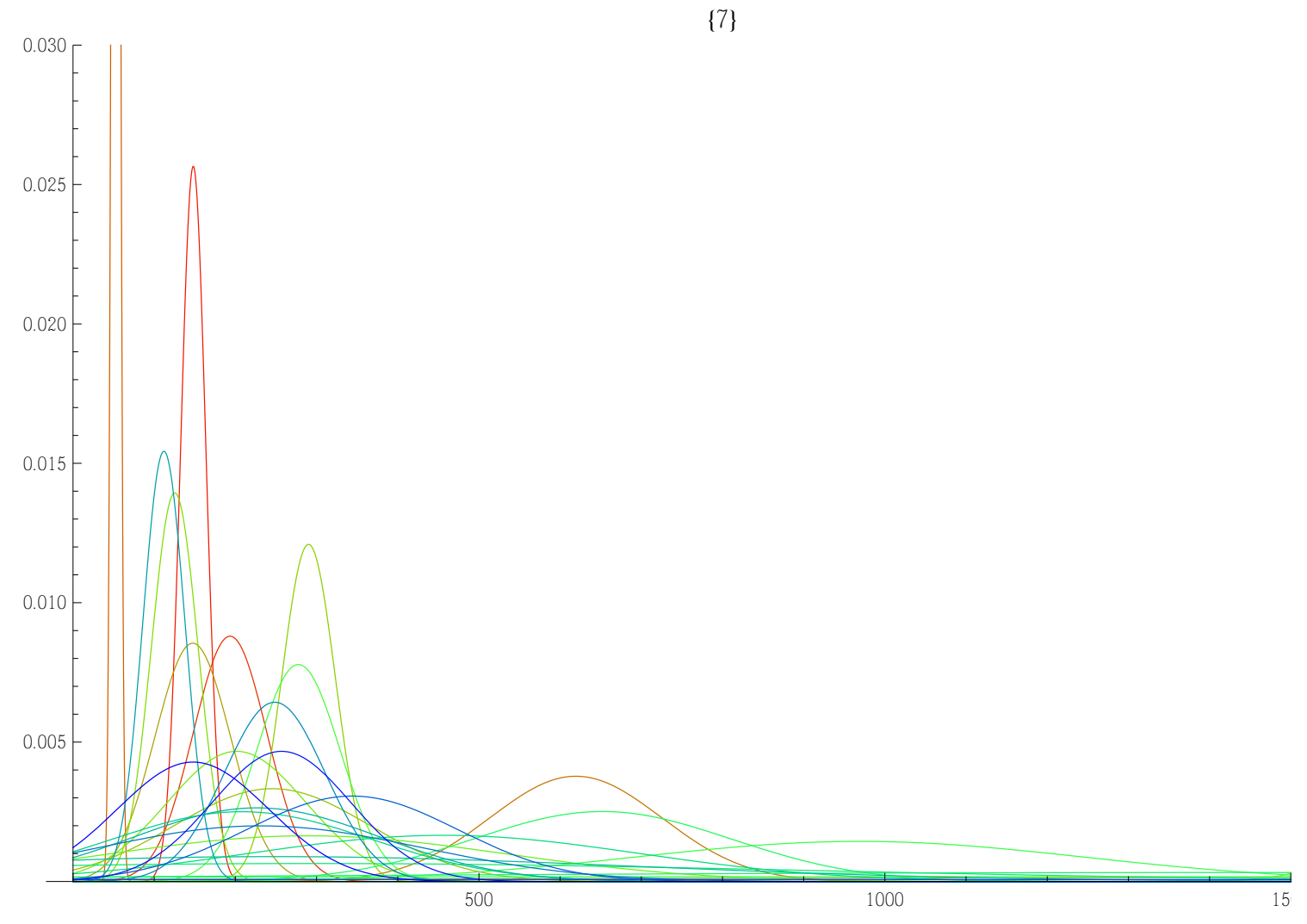


máÅN [UI SMMI MKMS]



The individual branches may be combined to obtain average times

$n M[\tilde{n} \mid I$ è $]$ ] $W=$ pìã [n[ñ èI à] / OVI \{àI NI PP\}] X



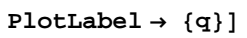


máÅO [NI UMMI MKMN]

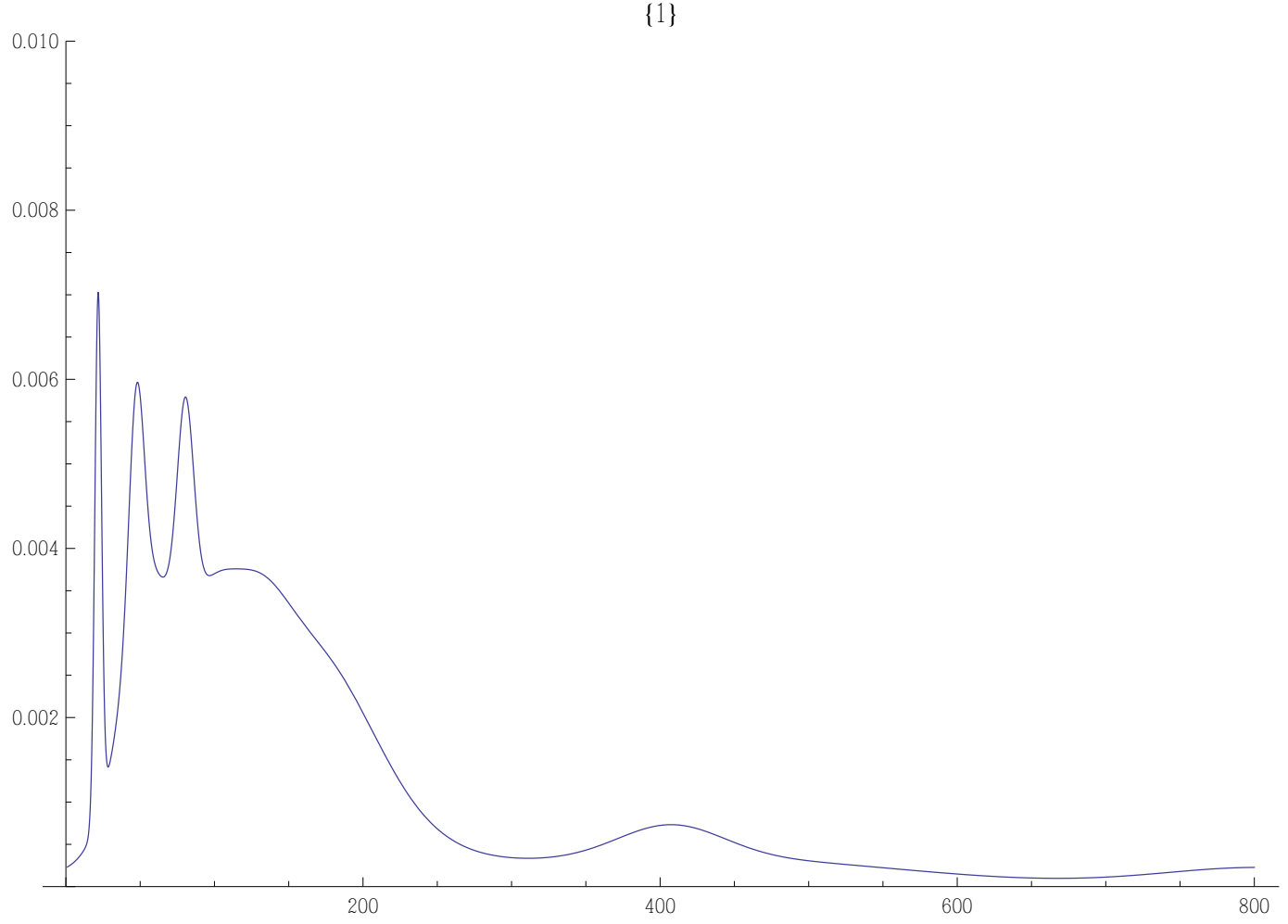

máĂO [OI OMMI MKMN]

$\{\mathrm{O}$

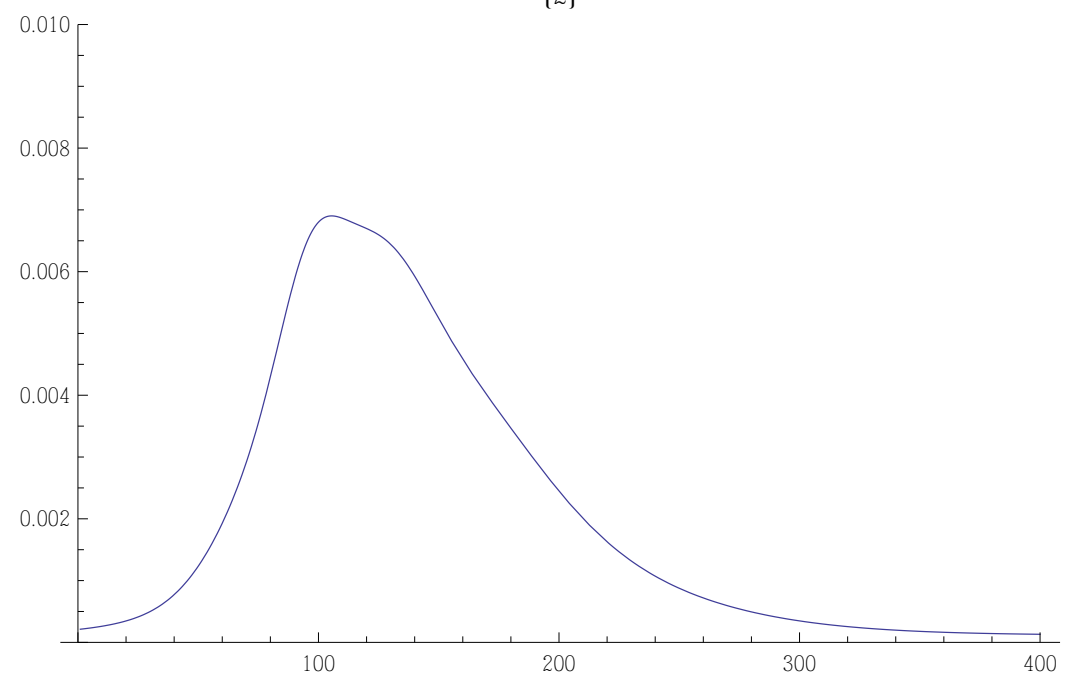


máÅO [PI QMMI KMN]

$\{\mathrm{P}\}$

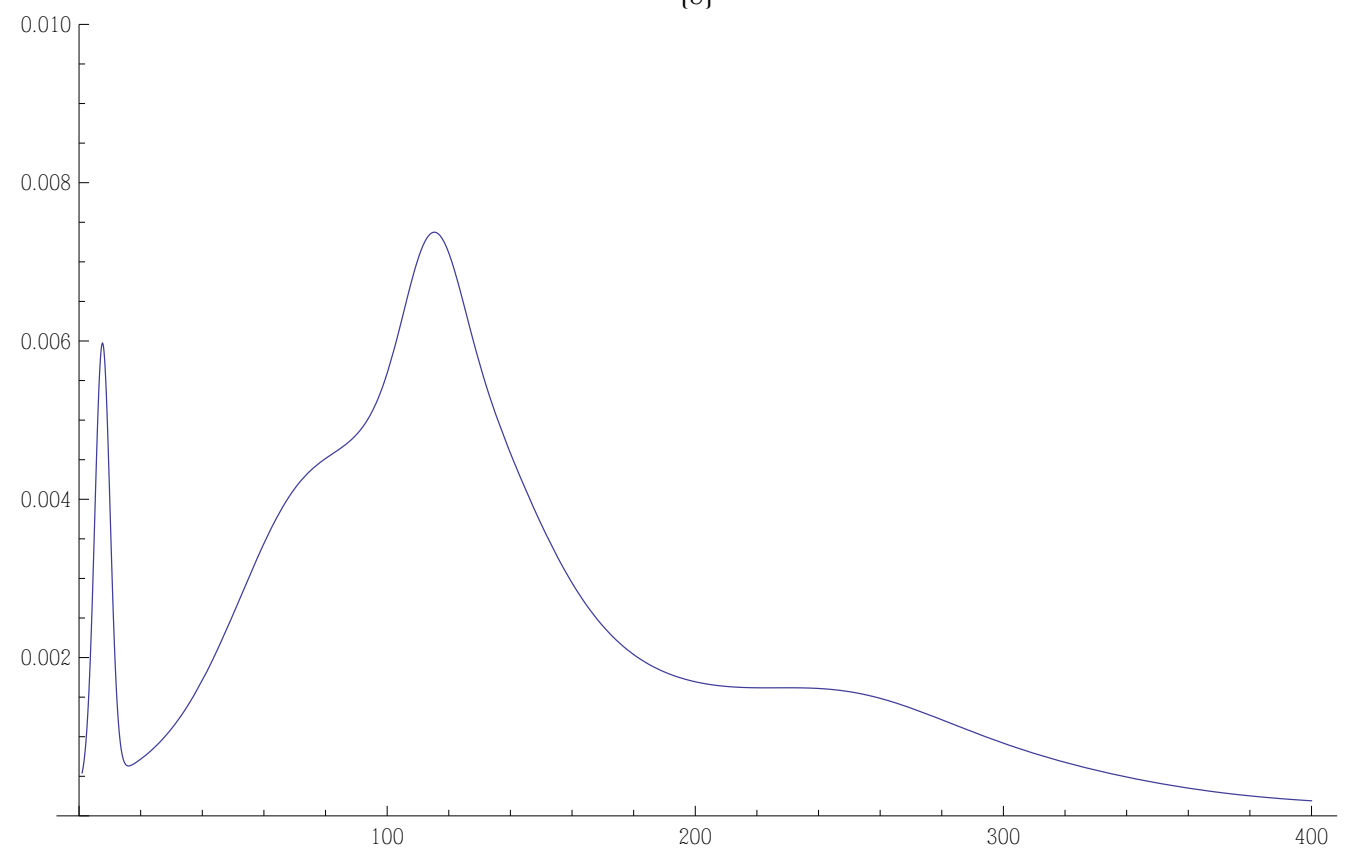

máĂO [QI PMMI KMN]




máÅO [RI PMMI KMNR]

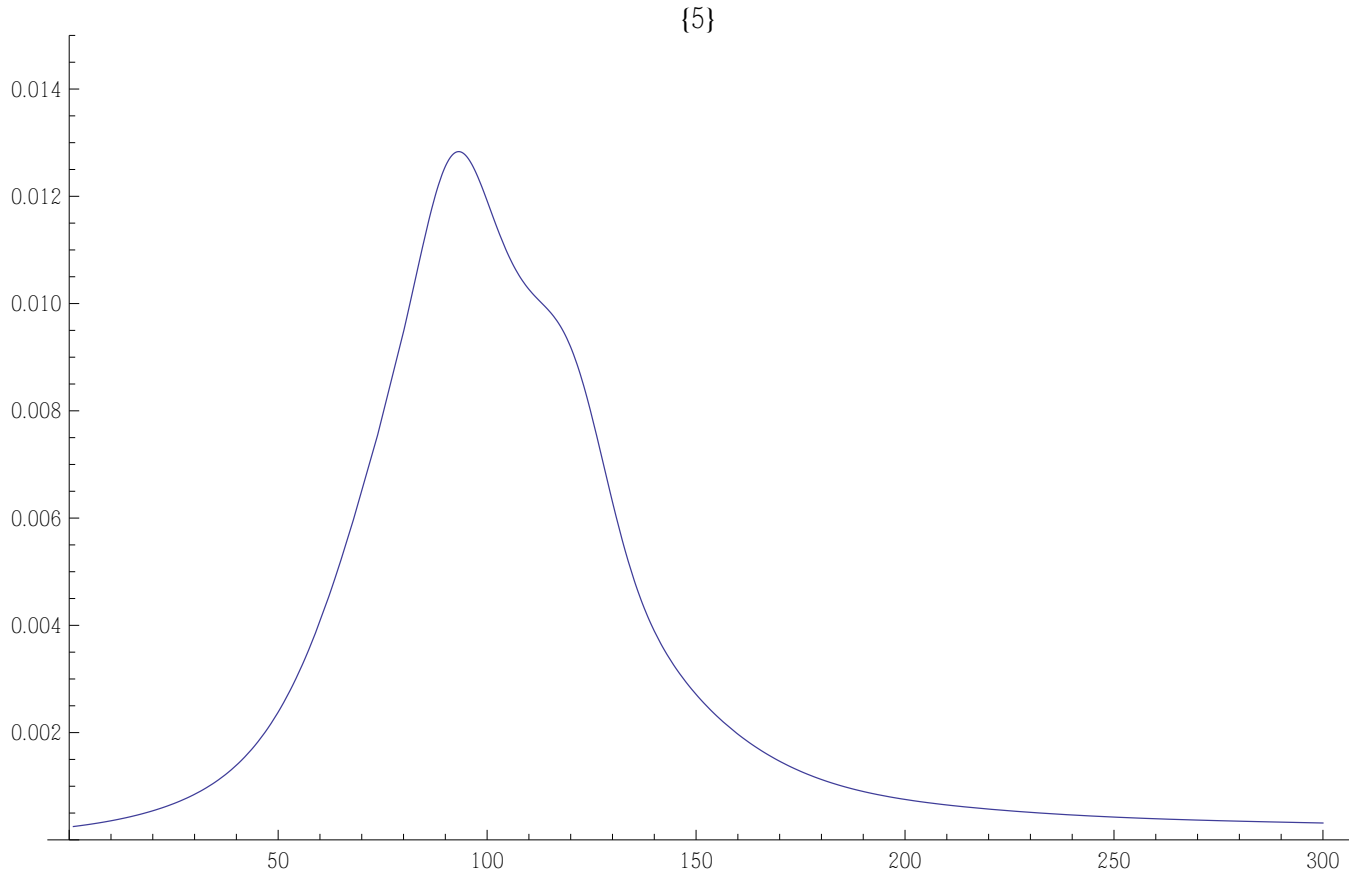

máĂO [SI PMMI KMN]

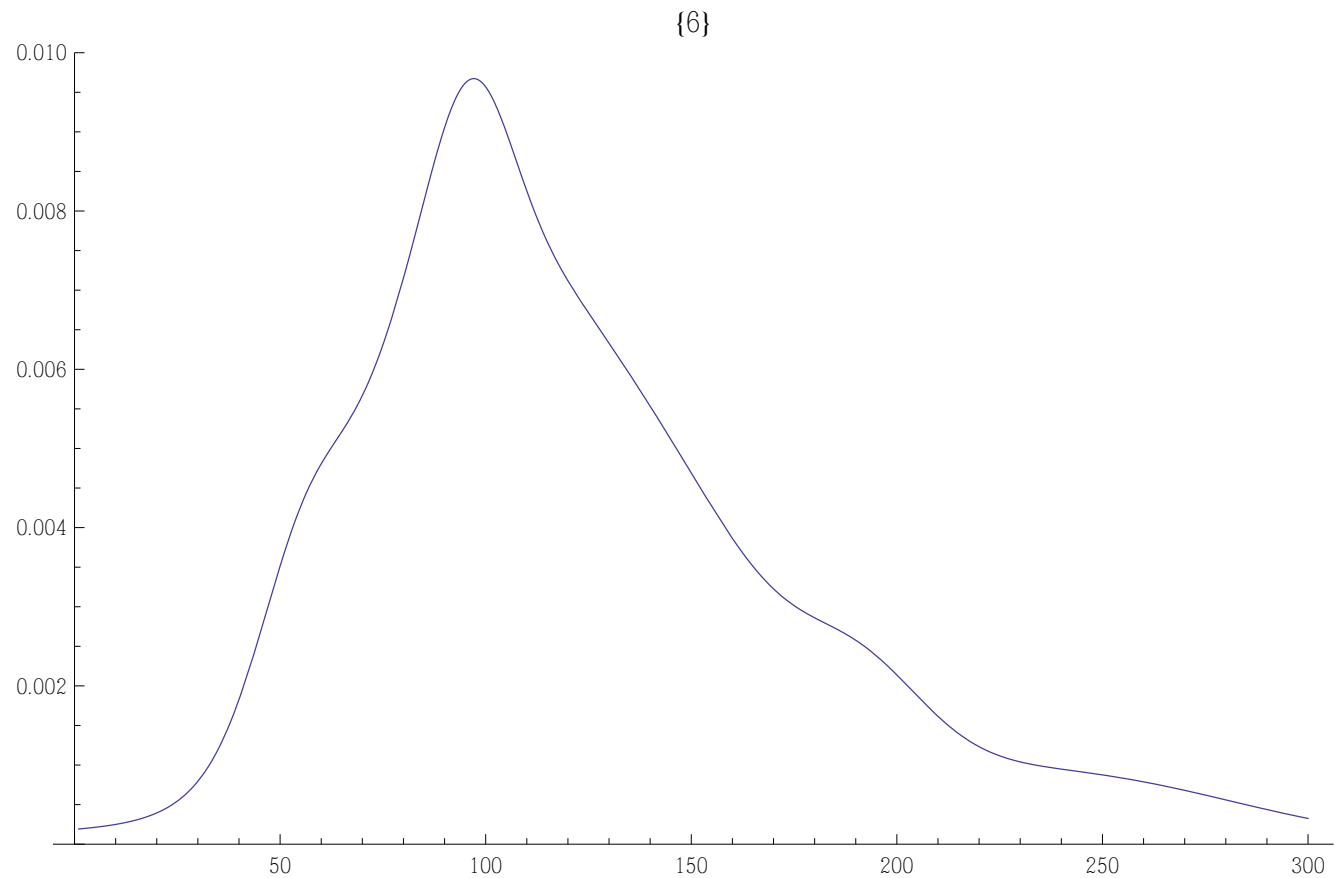


máÅO [TI UMMI KMMR]

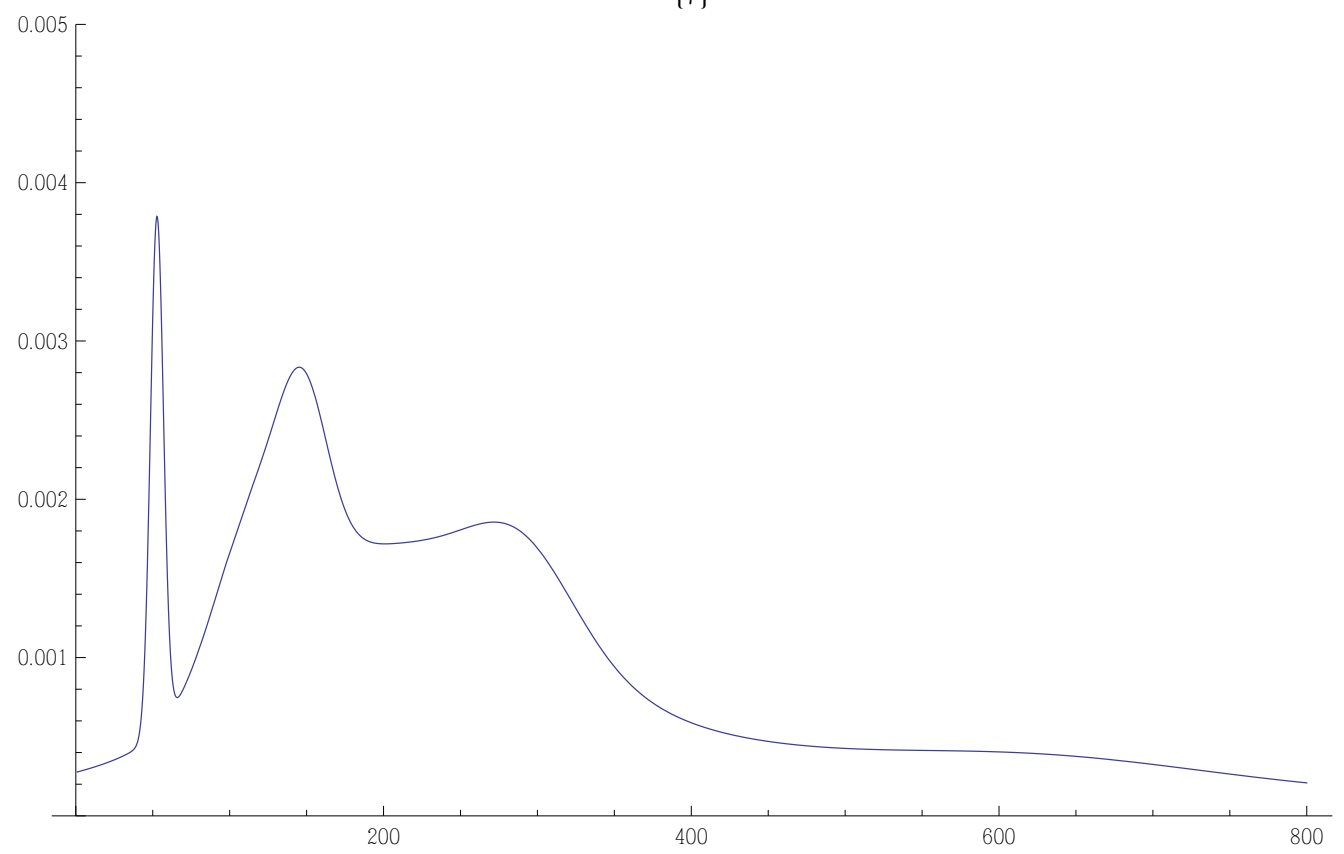

J2: Notice the spike at c50 generations ago, i.e. 650AD, the time of the Arab conquests.

máÅO [UI QMMI KMNR]

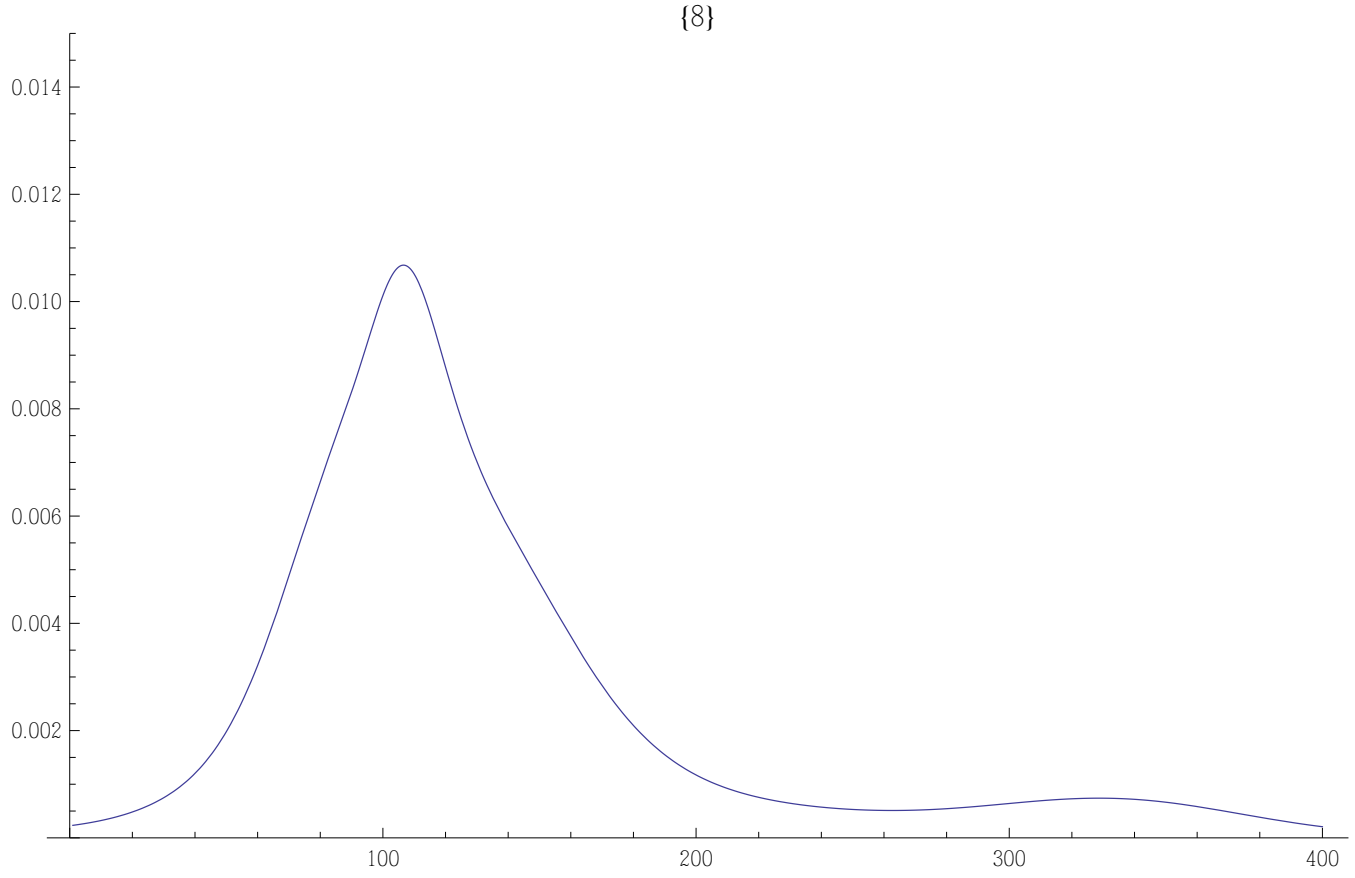

Some idea of the TMRCA is obtained by averaging, either the individual times or the overall, notice the discrepancy

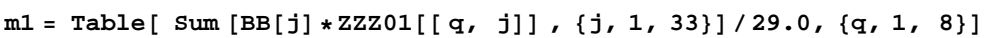

\{OSTKUQI OUUKQOOI NSSKRMQI NOOKVQI NNVKQNOI NOOKOVI RMNKUQOI NPPKMUQ\} 
OT * ãN

\{TOPNKSVI TTUTKPVI QQVRKSNI PPNVKPTI POOQKNPI PPMNKUQI NP RQVKTI PRVPKOT\}

$m M=q \sim \ddot{A} a ̈ E[$ kfåíÉÖê íÉ[ nM[ñI è]I \{ñI NI UMM\}]I \{èI NI U\}]

\{MKVMSURUI MKVOQPPI MKVPNQORI MKVRTNNOI MKVTQROUI MKVUVPSRI MKTRSRSOI MKVUSRUN\}

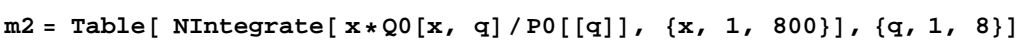

\{NTTKPOUI NUNKROPI NRPKVVOI NNOKVQPI NOPKVROI NOQKNSSI OTPKPOTI NPSKNOQ\}

OT * ão

\{QTUTKUSI QVMNKNOI QNRTKTVI PMQVKQTI PPQSKTNI PPROKQUI TPTVKUOI PSTRKPR\}

As the averages are not stable we use robust statistics, and stochastic simultations to get the MLE for the TMRCA

For later use we generate random quintiles at roughly $30,40,50,60,70 \%$

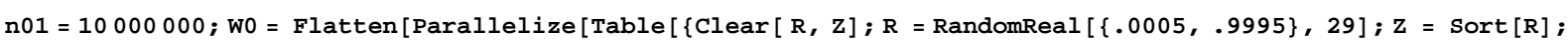
$\{w[[V]] I$ w[[NO]]I w[[NR]]I w[[NU]]I w[[ON]]\}\}I \{âI NI åmN\}]]I N]x $\{j E ́ \sim a ̊[t M] I \sqrt{s \sim e ̂ a ́ a ̊ a ̊ k ́[t M] ~}\}$

\{\{MKPMMNRRI MKQMMMUI MKQVVVTTI MKRVVURTI MKSVVTSV\}I \{MKMUOONVRI MKMUTVNPNI MKMUVTPPSI MKMUTUVUTI MKMUOONSP\}

Using the distribution of times ZZZ01 we use bootstrap to generate the same quintiles for our data, inc SD



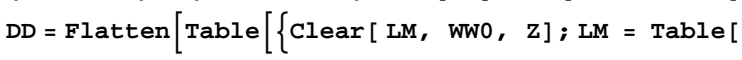

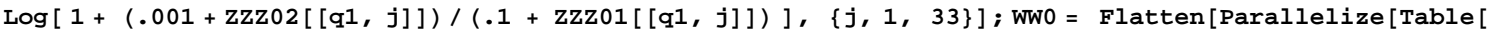

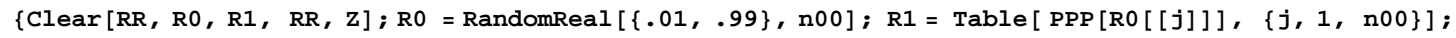

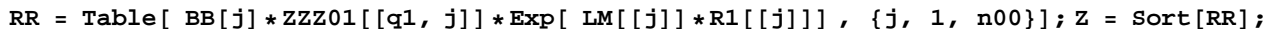
$\{\mathrm{w}[[\mathrm{NP}]] I \mathrm{w}[\overline{\mathrm{NS}}]] I \mathrm{w}[\mathrm{NV}]] I \mathrm{w}[[\mathrm{OO}]] \mathrm{I}$ w[[OR] $\}\} \mathcal{I}$ \{âI NI åmN\}]]I N]X

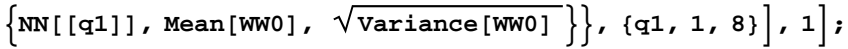

Thus for each file q our data used is the qunitile and its SD

j îêáñcçêã [aa ]

(NOON \{VMKUQVVI NNRKQPVI NQMKVRQI NTMKPPOI ONTKOVU\}

QSM \{NNQKUQUI NOUKSOQI NQPKTMTI NSOKQNQI NUVKOT\}

NOTM \{NMMKSUPI NNQKVURI NOVKPNUI NRMKMMUI NUSKNSQ\}

OUVU \{RVKOMPVI SVKVUTI UOKPPQOI VTKNVUSI NNSKPUU

NMOV \{UVKMMRRI VSKRSOQI NMQKUQOI NNQKPRVI NORKUUS

NRPP \{VOKRUQSI NMOKSSPI NNQKMQI NOTKTPUI NQQKRPO\}

NOQN \{NTSKSNPI OOPKNTSI OTOKUNSI PQMKMMVI RMMKSSV

VTN \{VUKQMOVI NMTKSI NNTKNMTI NOUKRUQI NQPKURR\}

\{NMKNPTPI NOKSONI NQKORSSI NTKSSNI OUKTTQN\} \{UKQQTVI VKOUSUI NMKUTOI NPKSPQNI NUKSRNS\} \{VKMSVOTI UKNPPVRI NMKMURTI NQKUQI OQKMRSP \{RKOTRMSI RKVOPUTI SKSTNNQI TKVUNQQI VKVNMOQ\} \{RKPNQOTI RKROVSUI SKNSNVTI SKSPVMNI UKMVNTT\} \{SKNMOORI SKRVORVI TKTQMSRI VKMMNVTI NMKSMVR \{OQKMUMPI OVKNVPNI PNKSRURI RPKQUPI VVKQSTU\} \{SKONSSUI SKNSTRPI TKMMSSNI UKRVVMUI NNKNOVN\}

This is now applied to each case, begiining with

We do G2a3 using 5 quintiles

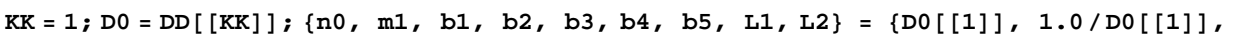
am[[OI N]]I am[[OI O]]I aM[[OI P]]I aM[[OI Q]]I aM[[OI R]]I aM[[OI O]] I NKR*aM[[OI R]] \}

\{NOONI MKMMMUNVMMNI VMKUQVVI NNRKQPVI NQMKVRQI NTMKPPOI ONTKOVUI NNRKQPVI PORKVQS

The stochastic simulation uses the branching distribution $\tau$ o to generate random quintiles. These are filtered by requiring they are within two SD of the data. To speed the process we compiled the branching distribution in files $29 \mathrm{ComFun}$ together with interpolation file W29ComFun which must be loaded. This information is contained in the function F4 used. This are the slowest part of the routine, taking about an hour. 


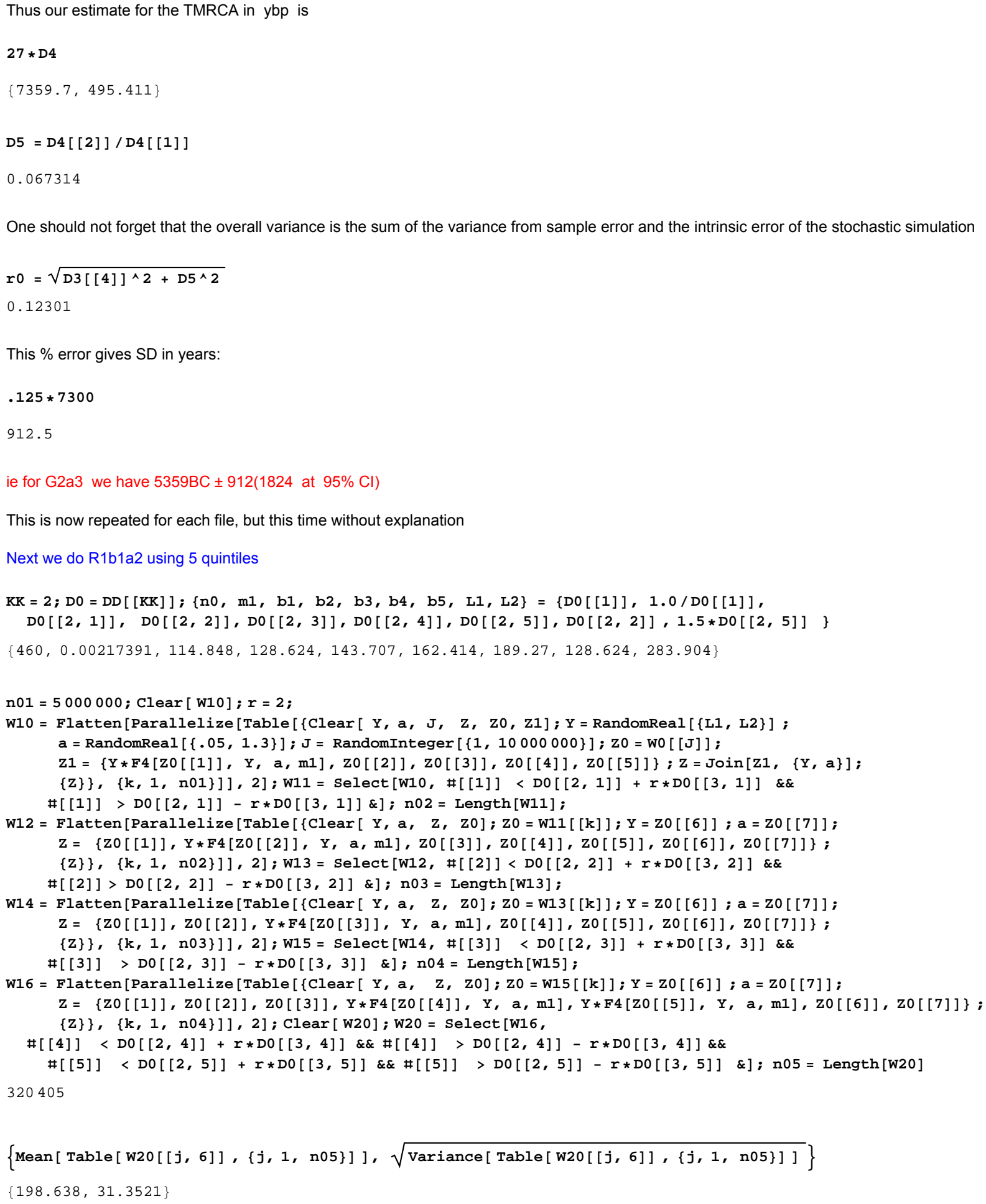




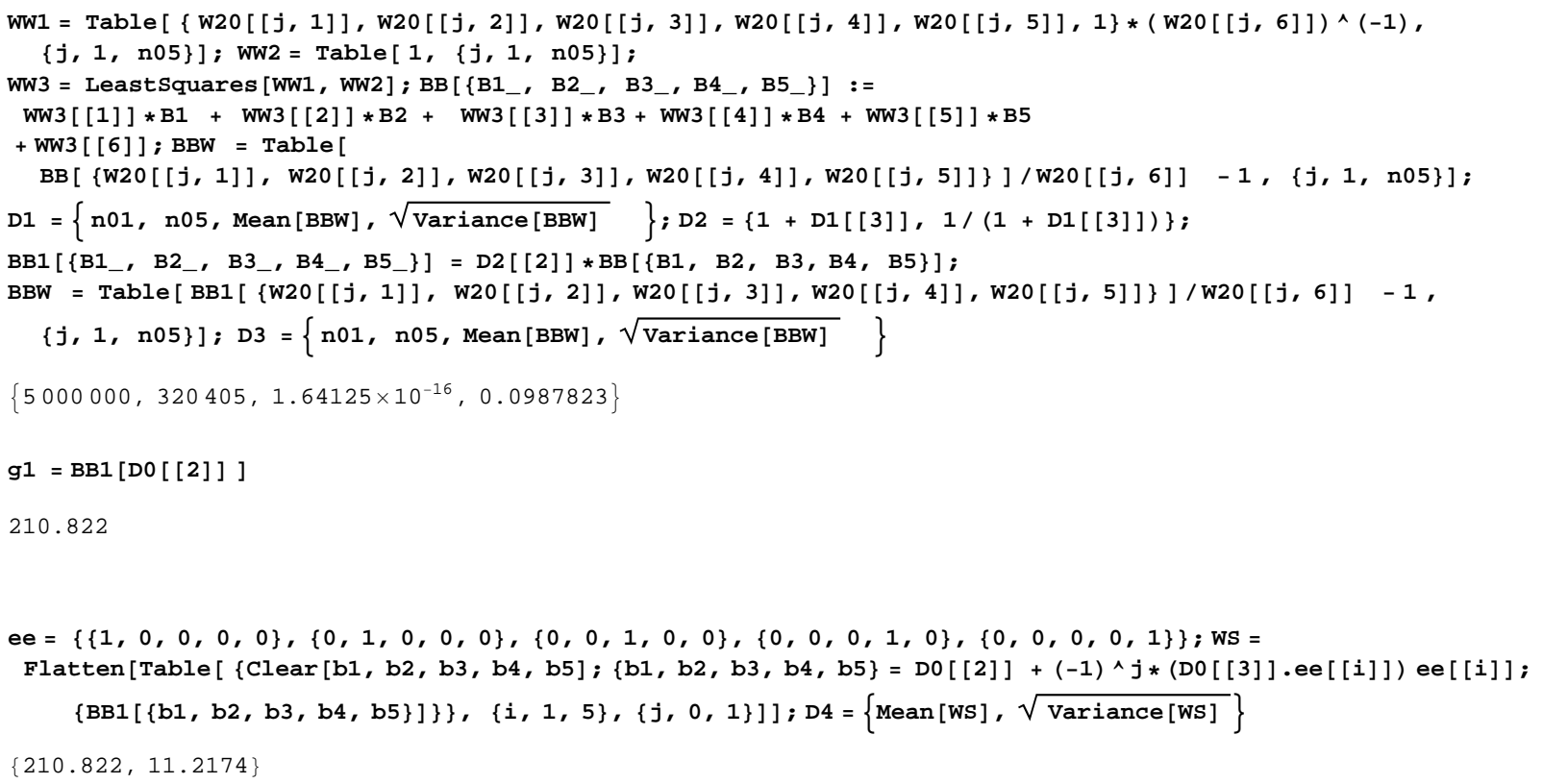




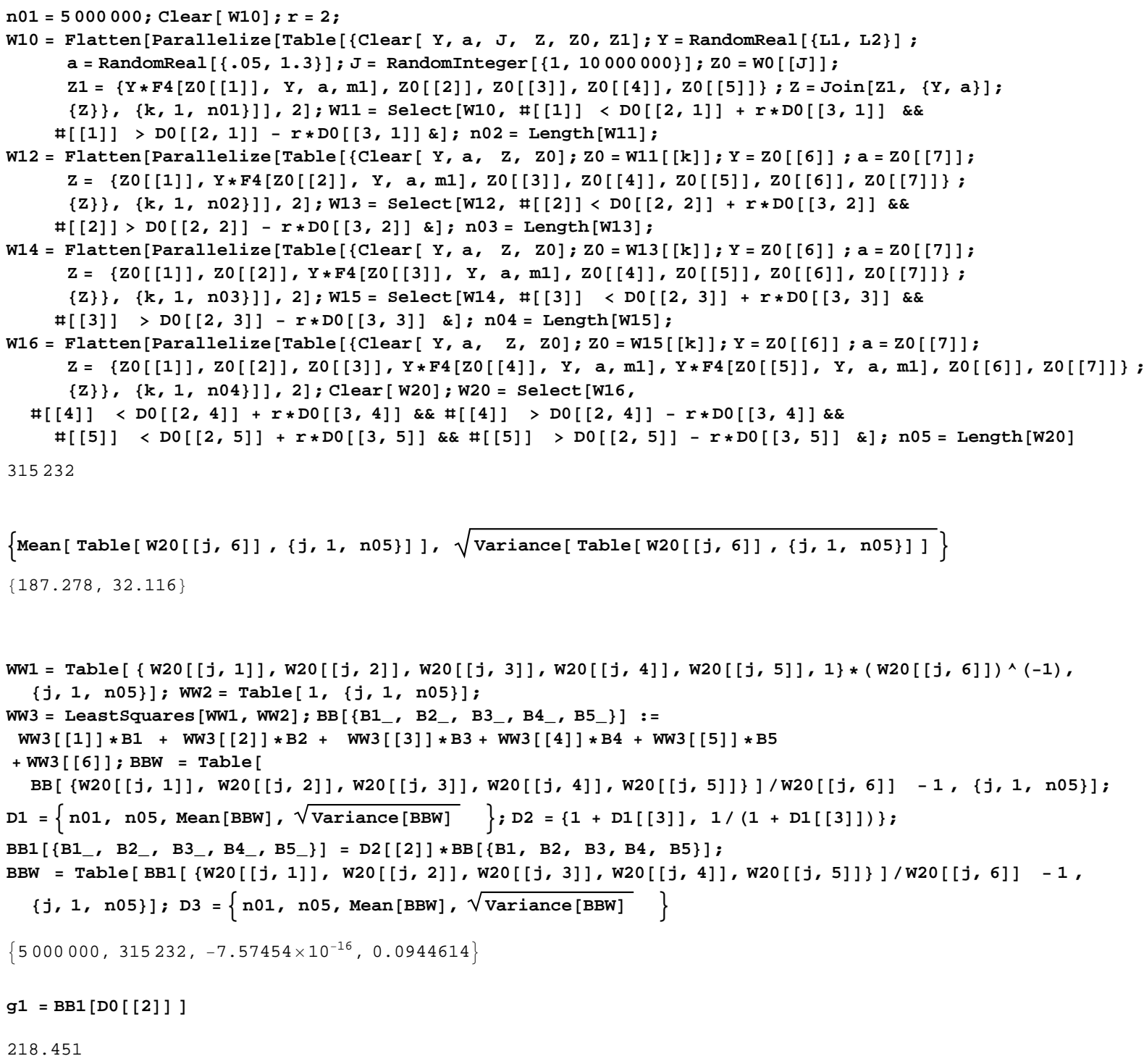




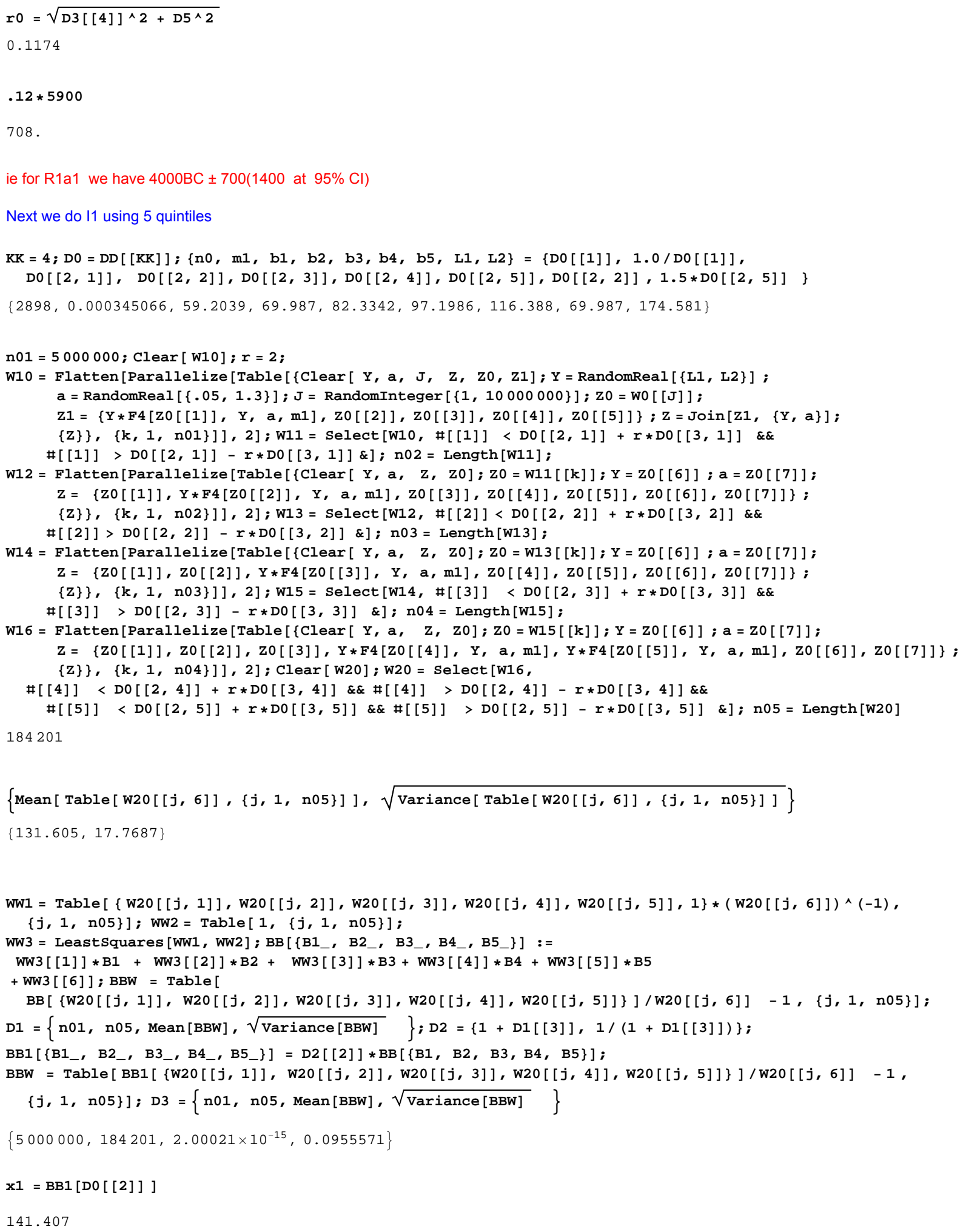


ÉÉ $=\{\{$ NI MI MI MI M $\}$ I $\{$ MI NI MI MI M $\}$ IMI MI NI MI M $\}$ IMI MI MI NI M $\}$ I $\{$ MI MI MI MI N $\}$ X tp $=$

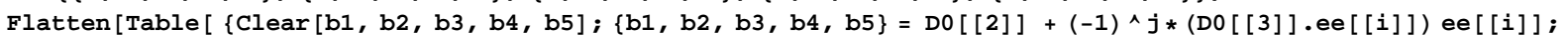

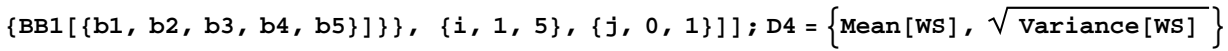

$\{$ NQNKQMTI SKRVPSR

$\tilde{\mathrm{nN}}=\mathrm{aQ}[[\mathrm{N}]]$

NQNKOMT

$\mathrm{OT} * \mathrm{aQ}$

\{PUNTKVVI NTUKMOU

$\mathrm{aR}=\mathrm{aQ}[[\mathrm{O}]] / \mathrm{aQ}[\mathrm{N}]]$

MKMOSSOUV

$\hat{\mathrm{e} M}=\sqrt{\mathrm{aP}[[Q]]\{O+\mathrm{aR}\{0}$

MKNMSPOT

KNN * PUMM

QNUK

ie for 11 we have $1800 \mathrm{BC} \pm 400(800$ at $95 \% \mathrm{Cl})$

Next we do L21 using 5 quintiles

$h h=R X a M=a a[[h h]] X\{a ̊ M I$ ãNI ÄNI ÄOI ÄPI ÄOI ÄRI iNI iO $\}=\{a M[[N]] I N K M / a M[[N]] I$ am[[OI N]]I aM[[OI O]]I am[[OI P]]I aM[[OI Q]]I aM[[OI R]]I aM[[OI O]] I NKR*aM[[OI R] \}

\{NMOVI MKMMMVTNUNTI UVKMMRRI VSKRSOQI NMQKUQOI NNQKPRVI NORKUUSI VSKRSOQI NUUKUP\}

åmN = R MMM MMMX “äÉ $\sim \hat{e}[t N M] X \hat{e}=O X$

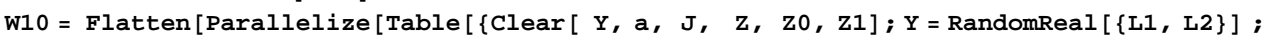

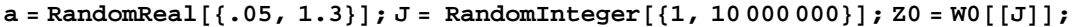

$\mathrm{wN}=\{\mathrm{V} * \mathrm{CQ}[\mathrm{wM}[[\mathrm{N}]] \mathrm{I}$ VI $\sim \mathrm{I}$ ãN]I wM[[O]]I wM[[P]]I wM[[Q]]I wM[[R]]\} Xw = gçåå[wNI \{vI $\sim\}] \mathrm{X}$

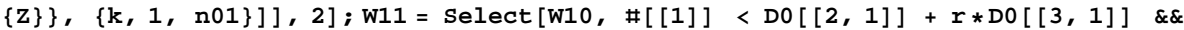
\#[[N]] > aM[[OIN]] - ê*aM[[PI N]] C]X åMO = iÉåÖİ̈̈[tNN]X

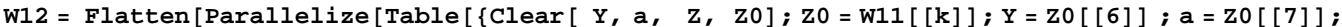
$\mathrm{w}=\{\mathrm{wM}[[\mathrm{N}]] I \mathrm{~V} * \mathrm{CQ}[\mathrm{wM}[[0]] I \mathrm{VI} \sim \mathrm{I}$ ãN]I wM[[P]]I wM[[Q]]I wM[[R]]I wM[[S]]I wM[[T]]\} X \{w\}\}I \{âI NI åMO\}]]I O]X tNP = pÉäÉÅí[tNOI \#[[O]]< aM[[OI O]] + ê*aM[[PI O]] CC

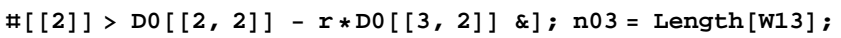

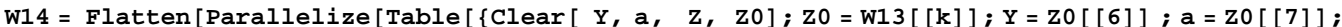
$\mathrm{w}=\{\mathrm{wM}[[\mathrm{N}]] I \mathrm{wM}[[0]] I \mathrm{~V} * \mathrm{CQ}[\mathrm{wM}[[P]] I \mathrm{vI} \sim \mathrm{I}$ ãN]I wM[[Q]]I wM[[R]]I wM[[S]]I wM[[T]] $\}$

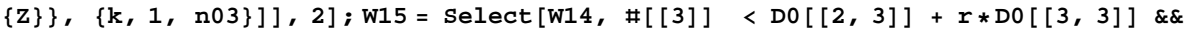
$\#[[P]]>a M[[O I P]]-\hat{e ̂} * a M[[P I P]] C] X$ åMQ = iÉåÖÍÜ[tNR]X

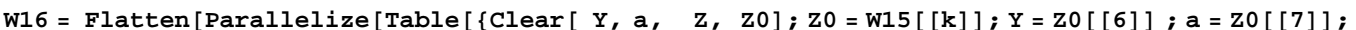

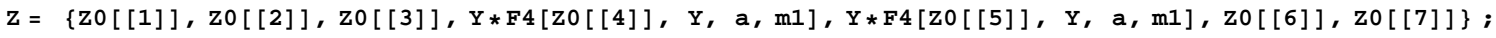
$\{w\}\} I$ \{âI NI åmo\}]]I O]X “äÉ ê [ tOM]X tOM = pÉäÉÅí[tNSI

$\#[[Q]]<a M[[O I Q]]+\hat{e} * a M[[P I Q]] C C \#[[Q]]>a M[[O I Q]]-\hat{e} * a M[[P I Q]] C C$ $\#[[R]]<a M[[O I R]]+\hat{e} * a M[[P I R]] C C \#[[R]]>a M[[O I R]]-\hat{e} * a M[[P I R]] C] x$ åmR = iÉåöíü[tOM] OPU PMU

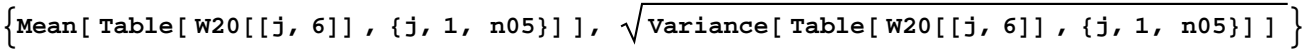
\{NPRKTPI NSKUNTN\} 


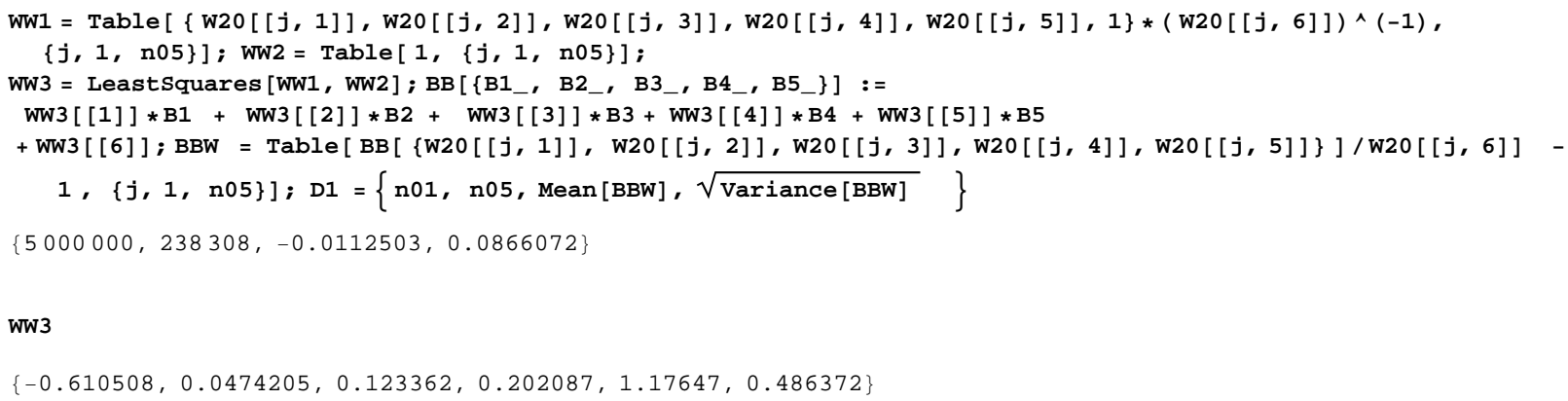




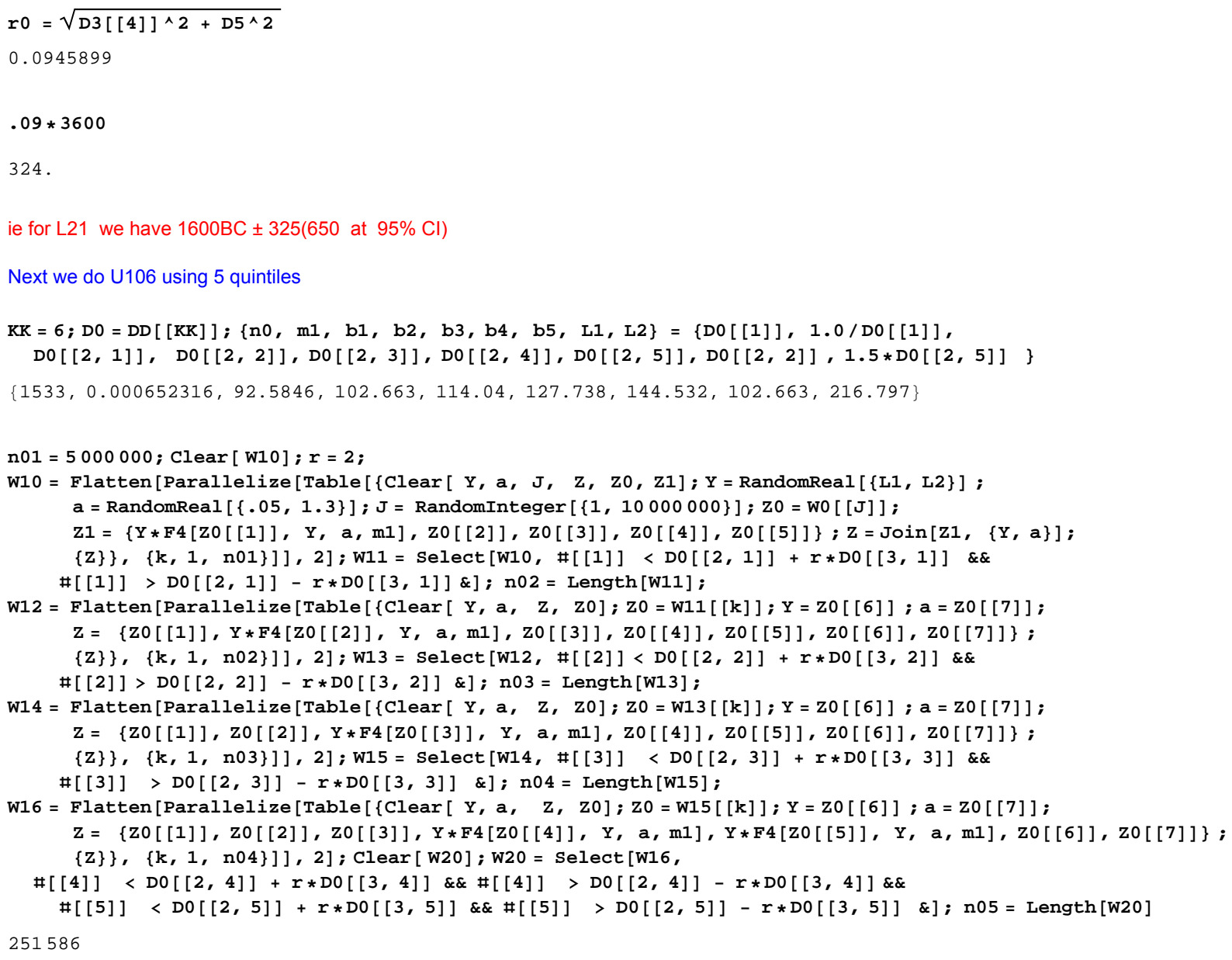


ÖN $=-N[\operatorname{am}[[0]]]$

NSOKTUT

ÉÉ $=\{\{$ NI MI MI MI M $\}$ I $\{$ MI NI MI MI M $\}$ I $\{$ MI MI NI MI M $\}$ I $\{$ MI MI MI NI M $\}$ I $\{$ MI MI MI MI N $\}$ X tp $=$

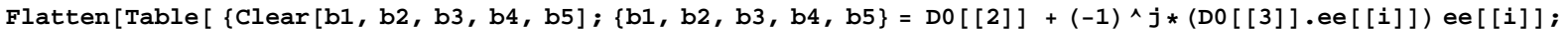

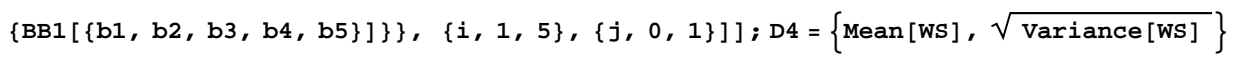
$\{$ NSOKTUTI SKUSTOR

$i \mathrm{~N}=\mathrm{aQ}[\mathrm{N}]]$

NSOKTUT

$\mathrm{OT} * \mathrm{aQ}$

$\{$ QPVRKORI NURKONS $\}$

$\mathrm{aR}=\mathrm{aQ}[[\mathrm{O}]] / \mathrm{aQ}[\mathrm{N}]]$

MKMQONURR

$\hat{\mathrm{e} M}=\sqrt{\mathrm{aP}[[Q]]\{0+\mathrm{aR}\{0}$

MKMVUUPTN

$\mathrm{KNM} * \mathrm{QQMM}$

QQMK

ie for $\mathrm{U} 106$ we have $2400 \mathrm{BC} \pm 440(880$ at $95 \% \mathrm{Cl})$

Next we do J2 using 5 quintiles

$h h=T X a M=a a[[h h]] X\{a ̊ M I$ ãNI ÄNI ÄOI ÄPI ÄOI ÄRI iNI iO $\}=\{a M[[N]] I$ NKM/aM[[N]]I aM[[OI N]]I aM[[OI O]]I aM[[OI P]]I aM[[OI O]]I aM[[OI R]]I aM[[OI O]] I NKR*aM[[OI R]] \{NOQNI MKMMMUMRUMOI NTSKSNPI OOPKNTSI OTOKUNSI PQMKMMVI RMMKSSVI OOPKNTSI TRNKMMP

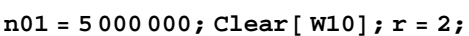

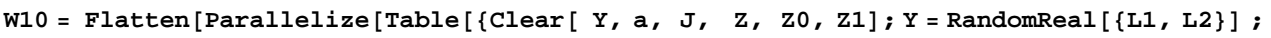

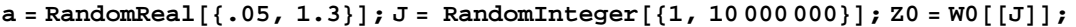

$\mathrm{wN}=\{\mathrm{V} * \mathrm{CQ}[\mathrm{wM}[[\mathrm{N}]] \mathrm{I}$ VI $\sim \mathrm{I}$ ãN] I wM[[O]]I wM[[P]]I wM[[Q]]I wM[[R]]\} X w = gçáå[wNI \{vI $\sim\}] \mathrm{X}$

$\{w\}\} I\{a ̂ I N I$ åm $\}]] I$ O]X tNN = pÉäÉÅí[tNMI \#[[N]]<aM[[OI N]] + ê*aM[[PI N]] CC

$\#[[N]]>a M[[O I N]]-\hat{e} * a M[[P I N]] C] X$ åmO = iÉåÖíü[tNN]X

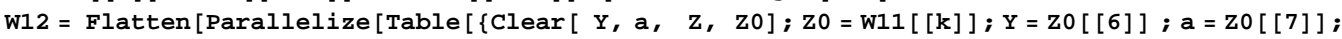
$\mathrm{w}=\{\mathrm{wM}[[\mathrm{N}]] I \mathrm{~V} * \mathrm{CQ}[\mathrm{wM}[[\mathrm{O}]] \mathrm{I}$ VI $\sim \mathrm{I}$ ãN]I wM[[P]]I wM[[Q]]I wM[[R]]I wM[[S]]I wM[[T]]\} $\mathrm{X}$ \{w\}\}I \{âI NI åMO\}]]I O]X tNP = pÉäÉÅí[tNOI \#[[O]]< aM[[OI O]] + ê *aM[[PI O]] CC \#[[O]] > aM[[OI O]] - ê*aM[[PI O]] C]X åmP = iÉåÖİ̈U[tNP]X

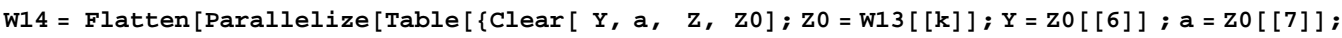
$\mathrm{w}=\{\mathrm{wM}[[\mathrm{N}]] \mathrm{I} \mathrm{wM}[[\mathrm{O}]] \mathrm{I} \mathrm{v} * \mathrm{CQ}[\mathrm{wM}[[\mathrm{P}]] \mathrm{I}$ VI $\sim \mathrm{I}$ ãN]I wM[[Q]]I wM[[R]]I wM[[S]]I wM[[T]]\} $\mathrm{X}$ $\{w\}\} I\{a ̂ I N I$ åMP $\}]] I$ O]X tNR = pÉäÉÅí[tNQI \#[[P]] < aM[[OI P]] + ê*aM[[PI P]] CC $\#[[P]]>a M[[O I P]]-\hat{e} * a M[[P I P]] C] X$ åMQ = iÉåÖÍÜ[tNR]X

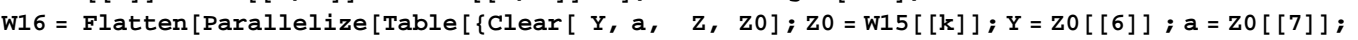

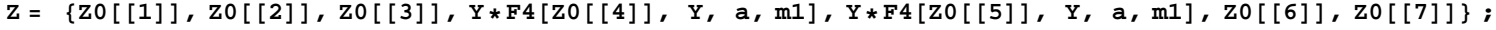
$\{w\}\} I$ \{âI NI åMQ\}]] I O]X “äÉ ê[ tOM]X tOM = pÉäÉÅí[tNSI

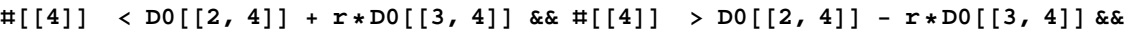

$\#[[R]]<a M[[O I R]]+\hat{e} * a M[[P I R]] C C \#[[R]]>a M[[O I R]]-\hat{e} * a M[[P I R]] C] x$ åmR = iÉåÖİ̈[tom] PPU TTP 


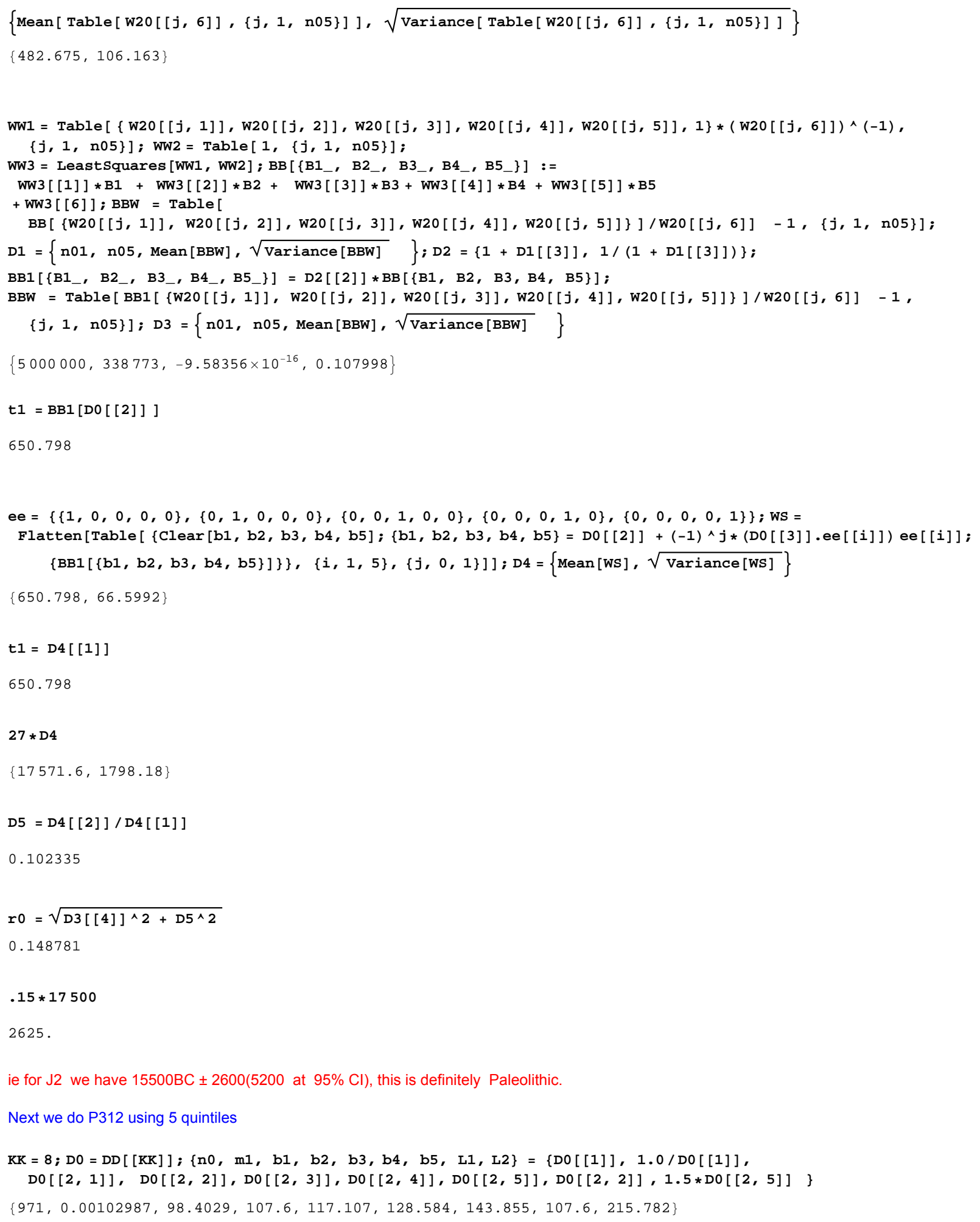




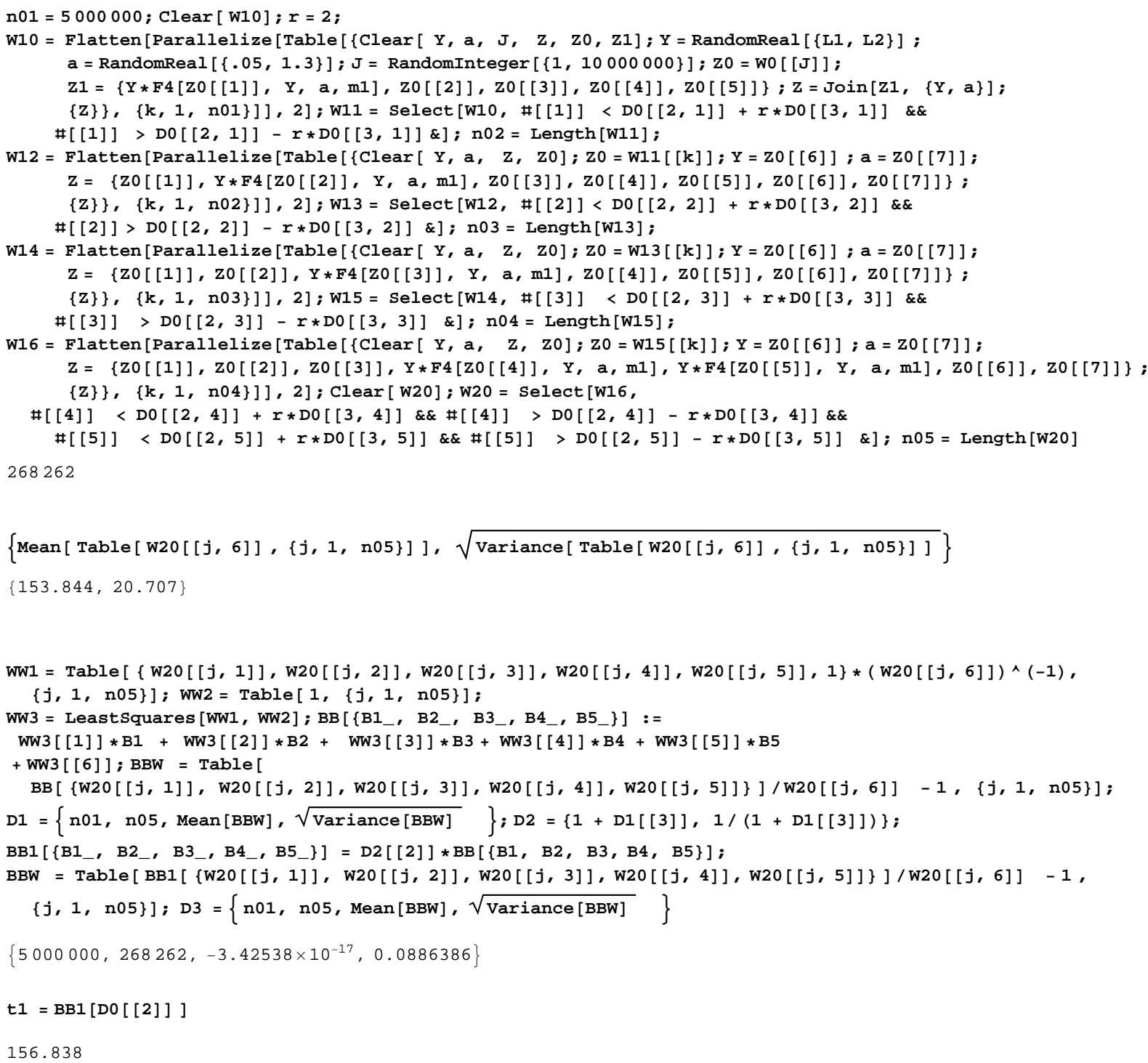




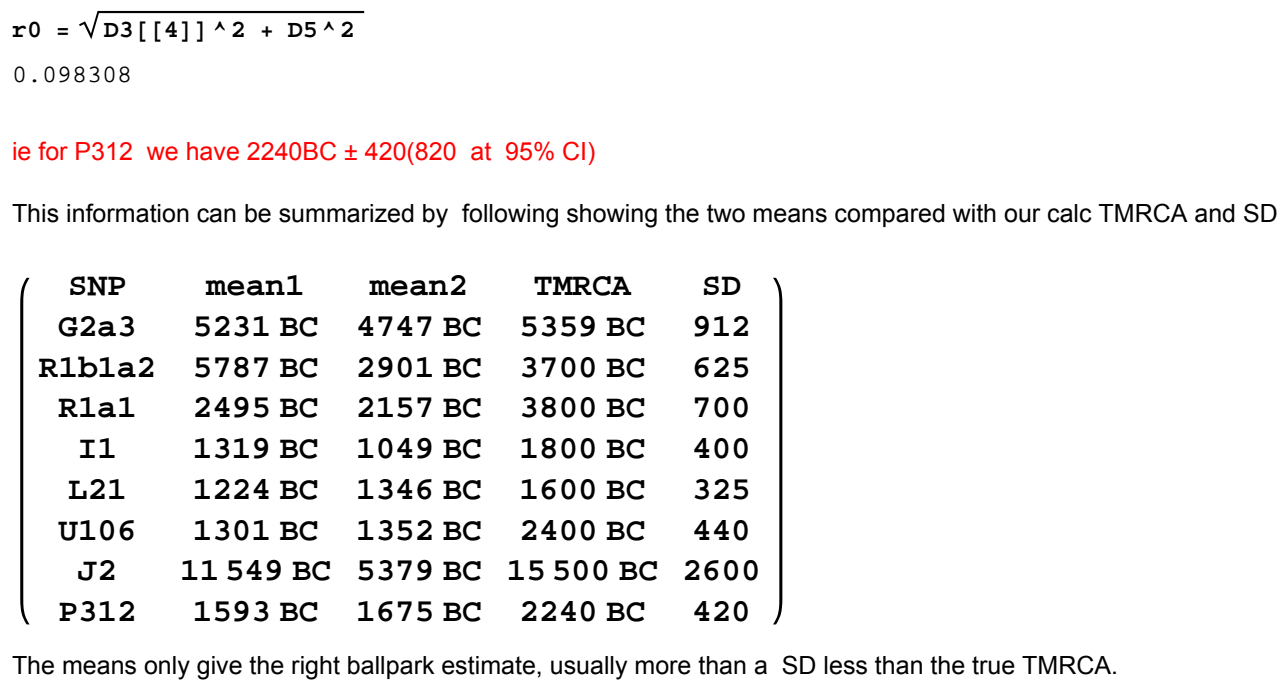

